\title{
Regulación del comportamiento durante la construcción conjunta de conocimientos en tareas cooperativas en entornos de aprendizaje virtuales asincrónicos y escritos
}

Autora: Denisse López Benavides

Tutora: Dra. Ibis M. Alvarez Valdivia

Seminario de Iniciación a la Investigación

Curso Académico 2009

Universitat Oberta de Catalunya

Barcelona, España

01.06.2009 


\section{Índice}

1. JUSTIFICACIÓN DEL ESTUDIO 1

2. MARCO CONCEPTUAL 2

2.1. COOPERACIÓN EN ENTORNOS DE APRENDIZAJE UNIVERSITARIO, VIRTUALES ASINCRÓNICOS Y ESCRITOS

2.2. CONSTRUCCIÓN DE CONOCIMIENTOS Y APRENDIZAJE COOPERATIVO EN ENTORNOS VIRTUALES ASINCRÓNICOS Y ESCRITOS: OPORTUNIDADES Y RIESGOS

2.3. COOPERACIÓN Y CORREGULACIÓN Y EN CSCL A TRAVÉS DEL DISCURSO DE BASE TEXTUAL

3.1. DISEÑO DE LA INVESTIGACIÓN

3.1.1. OBJETIVOS DE LA INVESTIGACIÓN

3.1.2. METODOLOGÍA EMPLEADA

3.2. PRESENTACIÓN DEL CASO

3.2.1. El ENTORNO DE APRENDIZAJE DE LA UNIVERSITAT OBERTA DE CATALUNYA (UOC) 16

3.2.2. PRESENTACIÓN DE LA SITUACIÓN DIDÁCTICA

3.3. PLANIFICACIÓN E IMPLEMENTACIÓN DEL TRABAJO DE CAMPO

3.4. VALORACIÓN Y DISCUSIÓN DE LA IMPLEMENTACIÓN DE LA PLANIFICACIÓN 21

3.5. ESTRATEGIA ANALÍTICA

3.6. EL ANÁLISIS DEL DISCURSO: PROCEDIMIENTO DE APOYO A LA EVALUACIÓN DEL DEBATE 24

3.6.1. PRIMER NIVEL DE ANÁLISIS: DESCRIPCIÓN DE LAS INTERACCIONES DURANTE TAREAS COOPERATIVAS EN ENTORNOS ASINCRÓNICOS VIRTUALES Y ESCRITOS

3.6.2. SEGUNDO NIVEL DE ANÁLISIS: CONTRASTACIÓN DEL PROCESO DE COOPERACIÓN CON LOS RESULTADOS DEL APRENDIZAJE

3.6.3. TERCER NIVEL DE ANÁLISIS: MODELOS DE REGULACIÓN DURANTE TAREAS COOPERATIVAS VIRTUALES

3.7. PROCEDIMIENTO PARA EL ANÁlisis de DATOS 27

3.7.1. PROCESO DE CODIFICACIÓN Y DE ANÁLISIS DE CONCORDANCIA ENTRE CODIFICADORES. 29

3.8. ANÁLISIS CRÍTICO DE LA METODOLOGÍA UTILIZADA 30

3.9. ASPECTO ÉTICOS 31

4. RESULTADOS Y DISCUSIÓN

4.1. ANÁLISIS 1: DESCRIPCIÓN DE LAS INTERACCIONES DURANTE TAREAS COOPERATIVAS EN ENTORNOS ASINCRÓNICOS VIRTUALES Y ESCRITOS

4.1.1. DIMENSIÓN DE LA REGULACIÓN SOCIAL

4.1.2. DIMENSIÓN DE LA REGULACIÓN COGNITIVA 35

4.2. EL COMPORTAMIENTO DE LA REGULACIÓN DURANTE LAS INTERACCIONES EN EL TRABAJO COOPERATIVO

4.3. ANALISIS 2: CONTRASTACIÓN DEL PROCESO DE COOPERACIÓN CON LOS RESULTADOS DEL APRENDIZAJE

4.4. ANÁLISIS 3: MODELOS DE REGULACIÓN EN TAREAS COOPERATIVAS EN ENTORNOS VIRTUALES ASINCRÓNICOS Y ESCRITOS 
5. CONCLUSIONES $\quad 51$

6. PERSPECTIVAS DE INVESTIGACIÓN

7. BIBLIOGRAFÍA

ANEXO A MAPA CONCEPTUAL TEORÍA DE VYGOTSKY

ANEXO B CRONOGRAMA DE TRABAJO

$\underline{\text { ANEXO C ENTREVISTA INICIAL A LA DOCENTE DE LA ASIGNATURA }}$

$\begin{array}{ll}\text { ANEXO D } & \text { ENCUESTA EN LÍNEA }\end{array}$

ANEXO E CONCORDANCIA DE CATEGORIZACIÓN ENTRE JUECES

ANEXO F CATEGORÍAS DEL ESQUEMA ARGUMENTATIVO DE TEXTO ESCRITO

EN TAREA COOPERATIVA* $\quad 69$

F.1. CRITERIOS De EVALUACión de TEXTOS ESCRITOS

F.2. EJEMPLO DE LA ELABORACIÓN DE IDEAS: IDEA ELABORADA

F.3. EJEMPLO DE LA ELABORACIÓN DE IDEAS: IDEA INCOMPLETA

F.4. EJEMPLO DE LA ELABORACIÓN DE IDEAS: IDEA NO ELABORADA

ANEXO G EL ENTORNO VIRTUAL DE LA UOC (UNIVERSITAT OBERTA DE CATALUNYA)

ANEXO H RESUMEN DE LAS CATEGORÍAS DE LAS REGULACIÓN Y SUS DESCRIPTORES. 72 


\section{Resumen}

En este estudio se analizan las estrategias de regulación utilizadas, por cinco grupos de estudiantes, durante la construcción conjunta de conocimiento en una tarea cooperativa en un contexto universitario virtual de comunicación asincrónica. La experiencia se desarrolló en un curso de un programa de máster en la que los estudiantes aplicaron la técnica de estudio de caso en el desarrollo de dos actividades con demandas cognitivas argumentativas apoyadas en la discusión y concretadas en un informe escrito. A partir de la revisión teórica y del estado actual de las investigaciones, se delimita el concepto de regulación en las dimensiones social y cognitiva que actúan de manera entrelazada en la construcción conjunta de conocimientos. Mediante una metodología de estudio de caso y a través del análisis del discurso, se identifican tres modos de regulación: auto-, externa y conjunta implicados durante tareas cooperativas. Se categorizan los tipos de habla usados durante las actividades analizadas y se describen las interacciones. Se evalúa el resultado de aprendizaje a través de los informes escritos de cada actividad y por último se identifican cuatro modelos de interacción que reflejan la regulación del aprendizaje en las distintas fases de trabajo cooperativo en el contexto estudiado.

Palabras clave: Regulación del aprendizaje, aprendizaje cooperativo, entornos de aprendizaje virtuales asincrónicos y escritos 


\title{
Behaviour regulation during joint construction of meaning in cooperative tasks within virtual written and asynchronous learning environments
}

\begin{abstract}
This study analyzes the regulation strategies applied by five groups of students, during the process of joint construction of meaning in a cooperative task in a virtual university within an asynchronous communication context. The study was performed within a subject taught at a Master in E-learning program in which students applied the technique of case study for solving two different tasks. These tasks were based on written argumentation, supported by virtual discussions and its completion was defined through a written report. Based on a review of the theory and the current status of research, the concept of regulation in its social and cognitive dimensions are delineated; where the dimensions interweave during the process of joint construction of meaning. Through a case study methodology and by means of discourse analysis three modes of regulation are identified: self-, external and co-regulation all present during cooperative tasks. Then the types of language involved in the process of joint construction of meaning are categorized and the resultant interactions described. Subsequently, the learning effects of the groups are evaluated through their written reports. Lastly, four models of interaction, that reflect the regulation of learning at different stages of cooperative work in a virtual asynchronous communication context, are identified.
\end{abstract}

Keywords: Learning regulation, cooperative learning, virtual asynchronous and written based learning environments 


\section{Introducción}

Tradicionalmente, las investigaciones sobre aprendizaje cooperativo han centrado el objeto de estudio en probar su eficacia en comparación con otras formas de aprendizaje como el competitivo o el individualista; comprobándose ampliamente sus ventajas y beneficios, no sólo sobre el rendimiento de los alumnos, sino también sobre su desarrollo social (Ej. Arvaja, Salovaara, Häkkinen y Järvelä, 2007). Pero estos efectos positivos no se producen de manera espontánea con sólo formar grupos de alumnos. Es necesario atender al funcionamiento del proceso de interacción y a los principales mecanismos responsables de su eficacia para poder apoyarlo, regularlo y estructurarlo debidamente.

Recientemente, las investigaciones han cambiado el objeto de estudio, interesándose más por identificar los factores mediadores y mecanismos interpsicológicos implicados en la eficacia del aprendizaje cooperativo (Ej. Casanova, Alvarez y Gómez, 2009; Durán y Monereo, 2005; Järvela, Jarvenoja, y Veermans, 2008; Salonen, Vauras y Efklides, 2005).

Sin embargo, aún cuando parece esencial la influencia de la calidad de la regulación social durante la resolución de tareas cooperativas, no se puede perder de vista la participación de variables más personales que también pueden explicar numerosas dificultades de coordinación, de ambigüedad en papeles, de conflictos personales, etc., que hacen que los resultados del trabajo del grupo no sean los esperados (Ej. Eby y Dobbins, 1997; Gibson, 2001; Pftaff y Huddeleston, 2003). Esta situación de aprendizaje se hace más compleja o adquiere particularidades en contextos virtuales de comunicación asincrónica.

Por esta razón se piensa que más allá de la identificación de los beneficios cognitivos y motivacionales que traen el participar en formas sociales de aprendizaje (Ej., Boakaerts y Minnaert, 2006; Vauras, Iiskala, Kajamies, Kinnunen, y Lehtinen, 2003), se hace necesario investigar la probable influencia de factores relacionados con la participación de la regulación del comportamiento durante la realización de tareas cooperativas presumiblemente facilitadoras de la construcción de conocimiento.

Con el fin de plasmar el desarrollo de la investigación, orientada al objetivo previamente especificado, se ha estructurado el presente trabajo en cinco secciones principales: el marco conceptual, el marco empírico, los resultados, las conclusiones y las perspectivas de la investigación. 
En el marco conceptual de la investigación se analizan diversos trabajos que de algún modo han realizado aportaciones relacionadas con el objeto de estudio que se focaliza. Como resultado de este análisis se extraen los presupuestos teóricos y se elaboran las hipótesis que guían la investigación.

La segunda parte corresponde al diseño empírico realizado, se formulan las interrogantes y se precisan los objetivos del estudio. Asimismo se describen las técnicas y procedimientos utilizados tanto para producir los datos como para su análisis.

Seguidamente se presentan y se discuten los resultados obtenidos, en el mismo orden en que fueron obtenidos a lo largo de la realización de la investigación en cinco fases.

Por último, se elaboran las conclusiones y se esbozan algunas perspectivas que, desde la valoración crítica del trabajo realizado, pueden mejorarlo y sobre todo, aplicarlo en las prácticas educativas para las que fue concebido. Además se incluyen las referencias bibliográficas y los anexos con documentos, gráficos y figuras, que aportan información complementaria al análisis de los resultados. 


\section{Justificación del estudio}

Esta investigación responde a la necesidad de poseer indicadores válidos y fiables para observar las habilidades y estrategias de cooperación de los alumnos en contextos universitarios virtuales de comunicación asincrónica. El principal objetivo de este trabajo es contribuir al esclarecimiento de indicadores que permitan su observación, con vista a promoverlos o evaluarlos a través de ayudas educativas ajustadas.

Más concretamente para esta investigación nos interesa observar los mecanismos que favorecen la corregulación desde lo cual se puede precisar algunos indicadores que expliquen las contribuciones de la cooperación al aprendizaje a la construcción de conocimientos. Se parte del presupuesto básico que para que tenga lugar la construcción conjunta de conocimientos en tareas cooperativas los estudiantes deben discutir ideas/conocimientos de modo más o menos crítico. Así el lenguaje que utilizan refleja la calidad de sus contribuciones tanto a la regulación de la necesaria interdependencia entre los integrantes del grupo como a la regulación de la construcción del conocimiento que demanda la tarea en cuestión (Ej. Bell, 1997; Belland, Glazewski y Richardson, 2008; Mercer, 2004).

Desde este punto de vista, la observación de la regulación social durante la construcción conjunta de conocimientos, ofrece una perspectiva de gran interés para explicar la dinámica y la naturaleza relacional de la participación en actividades de aprendizaje cooperativo. Además, la regulación social proporciona explicaciones para comprender la génesis del aprendizaje a nivel del sistema social, en este caso en el seno de un grupo de pares y a nivel individual (Ej. Bodrova, 2006; Järvela et. al., 2008; Salonen et. al., 2005). 


\section{Marco conceptual}

La universidad, idealmente, forma profesionales que pueden y deben trabajar en equipo. Aprender a trabajar de modo cooperativo se establece como una competencia básica en el actual currículo universitario. Aprender a colaborar y colaborar para aprender son dos actividades que van unidas en 1 os entornos presenciales y/o virtuales. A su vez las exigencias de innovación de la docencia universitaria derivadas de los planteamientos del Espacio Europeo de Educación Superior sitúan la enseñanza y el aprendizaje de las competencias en el centro mismo de la tarea docente y, en especial, la competencia de autorregulación del aprendizaje. Ambas cuestiones adquieren ciertas particularidades cuando se trata de un entorno de aprendizaje virtual, asincrónico y escrito.

En este apartado se presentan conceptos, enfoques y tendencias actuales de la investigación al respecto. El propósito de esta revisión teórica es esclarecer los mecanismos psicológicos que están en la base de la construcción de conocimientos en tareas de aprendizaje cooperativo con demandas argumentativas, en entornos de aprendizaje virtuales y asincrónicos. Particularmente interesa destacar el papel que juega la regulación del comportamiento de los estudiantes en este proceso.

Con el objetivo de ordenar las referencias y las ideas que se extraen para este análisis, en primer lugar se analizan los presupuestos conceptuales de la cooperación en entornos de aprendizaje universitario, virtuales asincrónicos y escritos. Seguidamente y en relación directa con ello, se revisan los planteamientos didácticos que prevalecen para este tipo de aprendizaje, especialmente las tareas con demandas argumentativas. Por último y con mayor interés por su importancia para esta investigación, se profundiza en estudios que han abordado la regulación del aprendizaje durante tareas cooperativas en el ámbito universitario.

\subsection{Cooperación en entornos de aprendizaje universitario, virtuales asincrónicos y escritos}

Un entorno virtual de aprendizaje no es cualquier sitio web educacional (educational web site) como tampoco es sinónimo de campos virtual (virtual campus). Entre otras especificidades tecno-pedagógicas, un entorno virtual de aprendizaje (EVA) debe estar diseñado de modo que los estudiantes no solo sean activos, sino también actores. Los estudiantes, mediante interacciones educativas, construyen y reconstruyen información que comparten en el entorno virtual de aprendizaje. Así el EVA es esencialmente un espacio social (Dillenbourg, Schneider y Synteta, 2002). 
La docencia en entornos virtuales educativos de educación superior, basados en la comunicación asincrónica y escrita, puede definirse como aquella experiencia planificada de enseñanza que utiliza una amplia gama de tecnologías para lograr alcanzar los objetivos de aprendizaje en el marco de un contenido o área del conocimiento particular (Badía y Alvarez, 2009).

En esta línea el trabajo colaborativo con apoyo tecnológico, en inglés, Computer Supported Collaborative Work (CSCW), ha tenido un avance muy rápido, por las propias necesidades sociales, creando herramientas específicas, y se puede considerar el antecesor inmediato del CSCL (Computer Supported Collaborative Learning). Tanto uno como otro responden a la evolución social y a 1os cambios en las formas de la división social del trabajo, que requieren la subdivisión de actividades de producción complejas y la consiguiente repartición de tareas entre diferentes actores, separados a veces por la distancia, y desempeñando formas especializadas de trabajo. En ese contexto, las capacidades o competencias a ser aprendidas se han modificado en algunos casos, requiriendo capacidades especializadas en el interior de un sistema de trabajo sino también, y muy especialmente, el aprendizaje del trabajo en equipo como forma habitual de desempeñar 1os aprendizajes logrados (Rodriguez Illera, 2001).

El CSCL en situaciones de comunicación asincrónica, tiene como base de comunicación e interacción, el lenguaje escrito. Es por esto que parece importante comprender el proceso de regulación durante la construcción conjunta de conocimiento a través del análisis del discurso. Este tipo de análisis permite profundizar en cómo se regulan entre sí los elementos de un grupo cooperativo en una situación de aprendizaje virtual. Por otro lado, parece fundamental tener en cuenta cómo se estructura la colaboración y cómo contribuyen los procesos de regulación a la aparición de interacciones productivas entre alumnos de modo que favorezcan la construcción conjunta de conocimiento y la propia auto-regulación (Onrubia, Colomina y Engel, 2007).

La investigación sobre CSCL, actualmente se preocupa por desarrollar maneras de aumentar las probabilidades de que ocurran interacciones entre los elementos del grupo que potencien verdaderamente la construcción de significados. Conocer, por ejemplo, cual es el tipo de discurso que estimula la construcción de conocimiento, se hace necesario para su mejor comprensión. 


\subsection{Construcción de conocimientos y aprendizaje cooperativo en entornos virtuales asincrónicos y escritos: Oportunidades y riesgos}

Los defensores del aprendizaje cooperativo siempre han resaltado sus efectos sobre la dinámica del grupo, la mayor integración y motivación de 1 os estudiantes, así como 1os propios resultados del aprendizaje. Sin embargo, menos atención se ha prestado a 1 os múltiples casos en 1 os que no funciona como se espera, o cuando se producen varios efectos no deseados.

Dillenbourg (1999) dejó ver cómo la noción misma de aprendizaje colaborativo no deja de ser un cajón de sastre en el que todo cabe. Aclara, entonces que el aprendizaje colaborativo es, ante todo, un tipo de situación. Sin embargo surge aquí otra problemática para su estudio: la situación de aprendizaje puede ser definida de diversos modos, con atención, por ejemplo, a un variado número de participantes, a tareas simples o complejas, breves o de duración semejante a un curso académico, colaborando o simplemente trabajando juntos, con medios y estrategias muy diferentes, o con formas de comunicación también variadas, compartiendo presupuestos culturales o simplemente unidos para esa situación concreta. Tal variación hace que sea muy difícil extrapolar resultados empíricos de un tipo de situación a otra, por más que todas sean denominadas colaborativas.

Otro rasgo a considerar muy especialmente en el diseño de entornos colaborativos es el carácter fundamental discursivo del conocimiento que se genera. Es decir, considerar la necesidad que tienen los participantes de acudir a la discusión verbal para coordinar las posiciones y significados que 1 os diferentes participantes otorgan a determinados aspectos de una actividad. Es evidente que toda actividad compleja requiere la negociación de los significados que los distintos sujetos tienen. Esta ha sido una de las maneras habituales de establecer entornos colaborativos: mediante herramientas que permiten comunicarse a los sujetos, compartir experiencias y puntos de vista, y discutir 1os resultados (Dillenbourg y Fischer, 2007).

Esta forma de colaboración se centra más en el proceso grupal de discusión que se realiza a distancia y supone una buena posibilidad para atenuar la ausencia de interacción cara a cara, teniendo incluso aspectos positivos en su forma asíncrona (Kang, 1998, Davies y Dalgarno, 2009). Algunos estudios han constatado favorables contribuciones de los entornos colaborativos específicamente diseñados para sustentar 1os aspectos discursivos de la colaboración (Davis y Brewer, 1997; Duffy, Dueber y Hawley, 1998; Shotsberger, 2001). 
El aprendizaje en ambientes colaborativos busca propiciar espacios en los cuales se dé la discusión entre los estudiantes al momento de explorar conceptos que interesa dilucidar o situaciones problemáticas que se desea resolver; se busca que la combinación de situaciones e interacciones sociales pueda contribuir hacia un aprendizaje personal y grupal efectivo. La preocupación del aprendizaje colaborativo gira en torno a la experiencia en sí misma, más que a los resultados esperados.

La teoría subyacente en estas investigaciones enfatiza el papel del diálogo como forma de colaboración privilegiada y proviene de los enfoques socioculturales. En general se constata el lugar del lenguaje como eje mediador de las actividades humanas, especialmente de aquellas que son interdependientes entre varios actores y exigen la comunicación lingüística como forma básica para llegar a acuerdos, como es el caso de las situaciones de aprendizaje cooperativo en entornos virtuales asíncronos y escritos.

Existe cierto consenso en afirmar que el uso educativo de los entornos virtuales basados en la comunicación asincrónica y escrita, en relación con las exigencias del aprendizaje auténtico, puede proporcionar diferentes posibilidades de diversa naturaleza a profesores y estudiantes.

Algunos autores (Ej., Osborn y Nelly, 2003), coincidiendo con los planteamientos originales de Wiggins (1990), definen cinco criterios específicos para ser tenidos en cuenta en el momento de planificar la evaluación auténtica de los aprendizajes en un curso desarrollado virtualmente: (1) presentar tareas reales y prácticas que puedan llevarse a cabo en un entorno virtual, (2) utilizar herramientas tecnológicas y recursos de contenido en formato digital que permitan compartir información y presentar conocimiento construido entre los estudiantes, (3) evaluar la actuación de los estudiantes, no sólo los conocimientos que puedan explicitar verbalmente, (4) proveer de manera sistemática una tipo de evaluación y feedback informativo a los estudiantes y por último, (5) garantizar la utilidad futura para los estudiantes de la tarea de evaluación.

Atendiendo a este planteamiento metodológico, parece ser que ese tipo de tareas de aprendizaje suponen una activa participación y diálogo (necesariamente asincrónico y escrito) entre el profesor y los estudiantes, y de éstos entre sí. A su vez, exigen la aplicación, por parte de cada estudiante, de las habilidades de síntesis, de pensamiento crítico y creativo, y de actitudes positivas hacia el intercambio con la materia y con los sujetos que participan de la actividad de estudio (otros estudiantes, el/la docente) conducentes a la construcción 
significativa del conocimiento en una comunidad de aprendizaje virtual (Ej., Gulikers, Bastiaens y Kirschner, 2004; Herrington y Oliver, 2000; Swan, Shen y Hiltz, 2006).

En esta línea el estudio de casos es una de las técnicas de aprendizaje cooperativo que mayor impacto ha tenido a nivel educativo, también en entornos virtuales. En esta técnica de aprendizaje auténtico, los estudiantes revisan un estudio de un escenario del mundo real, que usualmente se relacionen con los problemas o situaciones reales con los que el estudiante se puede encontrar. El estudio de casos es útil para presentar teorías y principios abstractos de forma que sean relevantes para los estudiantes (Casanova, 2008) además puede considerarse como un proceso pedagógico flexible y abierto, en el que las decisiones y soluciones a los problemas no están dadas a priori, sino que pueden cambiar en función de los supuestos teóricos y metodológicos, sirviendo de punto de partida a quienes realizan el estudio.

A su vez, los entornos de aprendizaje virtuales asincrónicos y escrito contienen ciertas coyunturas, incluso ventajas para la comunicación educativa que precisa el aprendizaje cooperativo. Duffy, Lowyck y Jonassen (1992), advierten tempranamente las ventajas de los foros electrónicos, en tanto permiten a los profesores observar las contribuciones de los alumnos a las discusiones, incluir las transcripciones de las discusiones en una carpeta para promover retroacción o evaluación, participar en discusión para modelar habilidades de pensamiento crítico, formular preguntas y comentarios para promover pensamiento crítico y proporcionar conocimientos cuando se requiera.

Sin embargo, son más recientes los intentos de ir más allá de la mera descripción de los mensajes en los foros de comunicación asincrónica, para entenderlos como una oportunidad para promover conocimiento y el aprendizaje. En este sentido, destacan el trabajo de Henry (1992) y las aportaciones de Garrison, Anderson, Archer y Rouke (2000).

La teoría subyacente en estas investigaciones enfatiza el papel del diálogo como forma de colaboración privilegiada y proviene de los enfoques socioculturales. En general se constata el lugar del lenguaje como eje mediador de las actividades humanas, especialmente de aquellas que son interdependientes entre varios actores y exigen la comunicación lingüística como forma básica para llegar a acuerdos, como es el caso de las situaciones de aprendizaje cooperativo en entornos virtuales asíncronos y escritos.

Precisamente este parece un aspecto de gran interés para el estudio de las estrategias que utilizan los estudiantes para la regulación de sus intervenciones durante el proceso de aprendizaje cooperativo en estos entornos. 


\subsection{Cooperación y corregulación y en CSCL a través del discurso de base textual}

El aprendizaje cooperativo involucra la coordinación de las interacciones sociales para trabajar juntos en la realización de una tarea (Johnson y Johnson, 1999). Para ello los estudiantes necesitan no solo interactuar, también pensar conjuntamente (inter think), combinar sus pensamientos de modo creativo, conseguir que la acción conjunta sea más que la suma de las partes. En este intercambio el lenguaje es el principal medio para la comprensión conjunta y para la negociación de las mejores soluciones a la tarea (Mercer y Littleton, 2007; Dawes, 2008; Wegerif, Mercer y Dawes, 1999; Wegerif, 2001).

La naturaleza correguladora de la participación en un grupo de trabajo ha sido remarcada por Rochelle y Teasley (1995) en su definición de trabajo colaborativo una actividad coordinada y sincrónica que es el resultado de una tentativa continua de construir y mantener una concepción compartida de un problema. El trabajo cooperativo virtual, según Barberà (2004) debe ser organizado de manera que la actividad de aprendizaje entre alumnos del mismo grupo solo se pueda hacer a través de la cooperación virtual. De esta manera, la única forma de comunicación, corregulación y construcción de conocimiento es la escritura.

Garrison y Anderson (2005) defienden que la comunicación de base textual posee atributos específicos que favorecen la reflexión y el discurso críticos. En esta línea Vygotsky considera al lenguaje como la herramienta principal para el desarrollo de procesos psicológicos, entre los que cabe mencionar el aprendizaje. Sin embargo, muchas de las herramientas tecnológicas usadas para apoyar el aprendizaje cooperativo en línea inicialmente no estuvieron diseñadas para favorecer la cooperación y la construcción conjunta de conocimiento (Onrubia, et al., 2007). Por otra parte, es importante atender al formato de la comunicación, especialmente cuando la comunicación escrita y asincrónica es el único vehículo de este proceso.

Tomando como base el enfoque sociocultural en relación con el CSCL desde el punto de vista de lo que se denomina 'implicación cognitiva' de los aprendices (learners) se enfatiza su vinculación con el establecimiento de una interdependencia genuina (Kang, 1998; Salomon, 1992). Es decir, la necesidad de que 1os aprendices implicados en el proceso de colaboración lo estén de tal manera que dependan unos de otros "genuinamente". Al respecto Salomon señala tres rasgos: 1) compartir los recursos (información necesaria, significados, concepciones y conclusiones), 2) división del trabajo entre los miembros del equipo (roles diferenciados y complementarios para un objetivo) y 3) compartir la cognición mediante una 
actividad conjunta realizada de manera explícita, de forma que pueda ser examinada, cambiada y elaborada por 1os compañeros.

Estos presupuestos conllevan cuidadoso diseño didáctico, de modo que se favorezca tanto la interdependencia como la efectiva construcción conjunta de conocimientos.

Existen diversas teorías que contribuyen a la comprensión del aprendizaje cooperativo soportado con tecnología con atención al enfoque constructivista y la regulación del aprendizaje durante tareas cooperativas. Para este estudio, por su amplio reconocimiento en los trabajos en la línea de investigación que se aborda, se consideran básicos los aportes de la teoría de Vygotsky, especialmente la concepción de la Zona de Próximo Desarrollo. En el anexo A se muestra un mapa conceptual que destaca los conceptos que conforman esta definición, concretamente la concepción del aprendizaje activo que tiene como soporte las mediaciones a través de las interacciones entre los sujetos que comparten la realización de actividades en contextos sociales situados. En estos intercambios el lenguaje juega un papel primordial, constituye la herramienta para expresar ideas, razonar, compartir y re-construir significados, en definitiva, para conocer y aprender desde la participación activa.

El elemento clave que rescatamos de este enfoque es la concepción del entorno o contexto de aprendizaje. Partiendo de la concepción vygotskiana, el contexto y las interacciones sociales hacen referencia a la ley genética que preconiza que el desarrollo de las funciones psicológicas superiores ocurre primero en el nivel interpersonal y después en el plano intrapersonal (Vygotsky, 1979). Asimismo es importante recuperar la idea de que el aprendizaje despierta una serie de procesos evolutivos internos, capaces de operar solo cuando estamos en interacción social.

Para Vigotsky el aprendiz requiere la acción de un agente mediador para acceder a la zona de próximo desarrollo, éste será responsable de ir tendiendo un andamiaje que proporcione seguridad y permita que aquél se apropie del conocimiento y lo transfiera a su propio entorno. El conocimiento propio que es discutido en grupo, motiva la construcción de nuevo conocimiento.

Sobre esta base y con el interés de explorar los modos de regulación de las interrelaciones durante la tarea de aprendizaje cooperativo, algunas investigaciones toman como unidad de análisis el estudiante como individuo y se proponen identificar su proceso personal de autoregulación (Whipp y Chiarelli, 2004), otras se refieren a varios aspectos del proceso de regulación que se establece entre los elementos del grupo (corregulación), pero en contextos 
no virtuales (Salonen et al., 2005; Volet, Summers y Thurman, 2008). En cualquier caso, independientemente del tópico que se focalice, para estos estudios es imprescindible considerar las particularidades del contexto, tanto en relación con la naturaleza de los intercambios que ocurren como en relación con el propio proceso de regulación del aprendizaje y a los formatos comunicativos en los que transcurre.

La noción de construcción de conocimiento, es una noción básica para integrar los aspectos cognitivos y de interacción del aprendizaje colaborativo, y se encuentra estrechamente relacionada con la nociones de colaboración y corregulación. El proceso de construcción conjunta de conocimientos requiere más que la simple participación de los estudiantes en una actividad de aprendizaje conjunta, puesto que el resultado de sus interacciones no siempre puede garantizar que se produzca un efecto de aprendizaje colaborativo y constructivo. Las observaciones realizadas durante los estudios de Reusser (2001) y Roschelle y Teasley (1995) en tareas cooperativas grupales, señalan que el compromiso mostrado por los estudiantes en la actividad conjunta era algunas veces divergente y que más adelante convergía.

En las situaciones educativas diseñadas bajo los principios constructivistas del aprendizaje, que adjudican a las interrelaciones un papel sustancial (Ej. Belland et al., 2008; Monereo \& Pozo, 2003; Hung y Der-Thanq, 2001) se pueden esperar diferentes formas de regulación de la actividad de aprendizaje que deben realizar los estudiantes para responder a las demandas sociocognitivas que les plantea estos diseños. El término de "regulación externa", por ejemplo, se aplica a situaciones en las que surge un desnivel de comprensión de algún segmento particular de la tarea, por parte de los integrantes del grupo. Quién realiza la regulación externa, es el que se siente mejor familiarizado con ese segmento y quién adopta un papel instructivo con el fin de orientar a los demás a comprender el segmento y así equilibrar el nivel de comprensión grupal. De manera alternativa, se encuentra la "regulación compartida" que se refiere a la regulación constante y vigilada de la actividad conjunta, la cual no puede reducirse a una simple actividad individual.

Algunos autores en esta línea sostienen que la colaboración y la co-construcción pueden ocurrir con poca frecuencia o no existir en los episodios de interacción de trabajo en grupo. Estos episodios pueden abarcar la mayor parte de las interacciones de los grupos, o puede presentarse de manera momentánea y discontinua a los largo del trabajo en grupo. Por esta razón, para identificar instancias del aprendizaje colaborativo, es necesario ir más allá de una categorización general de las interacciones emergentes en las tareas colaborativas y de co- 
construcción y delimitar episodios de interacción específicos que cumplan con una base conceptual de criterios sólida. (Ej. Vauras et al., 2003).

La autorregulación del aprendizaje comprende muchos aspectos que han sido estudiados de manera separada y esta tendencia parece continuar. Comprendida como un proceso activo, cíclico, recurrente, que involucra la motivación, la conducta y el contexto, es realmente un reto para la investigación, en la práctica, se dificulta abarcarla en su integridad. Sin embargo para esta investigación compartimos este punto de vista en la medida que parece imprescindible tener en cuenta la participación de la SRL (Self Regulated Learning) tanto a la regulación social como a la regulación de la construcción conjunta del conocimiento durante la realización de tareas cooperativas.

La realización de acciones con base en metas intrínsecas, el pensamiento crítico, el control de las autorreferencias, la elaboración y la solicitud de ayudas de otros compañeros, resultan estrategias de autoregulación utilizadas con mayor efectividad por los estudiantes durante tareas cooperativas en entornos virtuales de aprendizaje. Por el contrario, la planificación y el monitoreo de su actuación durante la realización de la tarea cooperativa se observan con menor frecuencia (Ej., Mauri, Colomina y Gispert, 2009; Winters y Costich, 2008). Los estudiantes que tienen mayores logros académicos también utilizan mejores estrategias para autorregular su aprendizaje en CSCL. Del mismo modo intervienen los conocimientos previos. Asimismo las caracerísticas de la tarea también pueden explicar las estrategias que utilizan los estudiantes (Ej., Vighnarajah, Wong, Su Luan y Abu Bakar, 2009).

En el contexto de actividades de aprendizaje dirigidas por estudiantes, el proceso de regulación también puede oscilar entre situaciones en las que una persona se encarga de suministrar temporalmente información al grupo o de adoptar un rol instructivo, y entre situaciones metacognitivas y co-reguladoras más equilibradas en las que se ven involucrados varios miembros del grupo (Ej. Volet et al., 2008).

Es posible establecer algunas aproximaciones conceptuales entre los procesos de autorregulación y los de corregulación. Así, para el análisis relacionado con la identificación del lenguaje de la corregulación en general se definen tres dimensiones: Motivación/Afecto, Cognición y Metacognición y Contexto/Tarea. A su vez se establece una correspondencia entre estas dimensiones y las dimensiones del discurso del trabajo cooperativo.

De este modo, a la vez que se estudia el proceso de cooperación (para lo cual resultan útiles las dimensiones del discurso cooperativo) se está estudiando el proceso de corregulación que 
pueda tener lugar a través de la observación de las cuatro fases de construcción de sentido en un grupo colaborativo (inicio, intercambio, negociación y aplicación. Las características de estas fases se encuentran explicitadas en el estudio de Casanova (2008). En la figura (Fig. 1) se representa la correspondencia conceptual establecida.

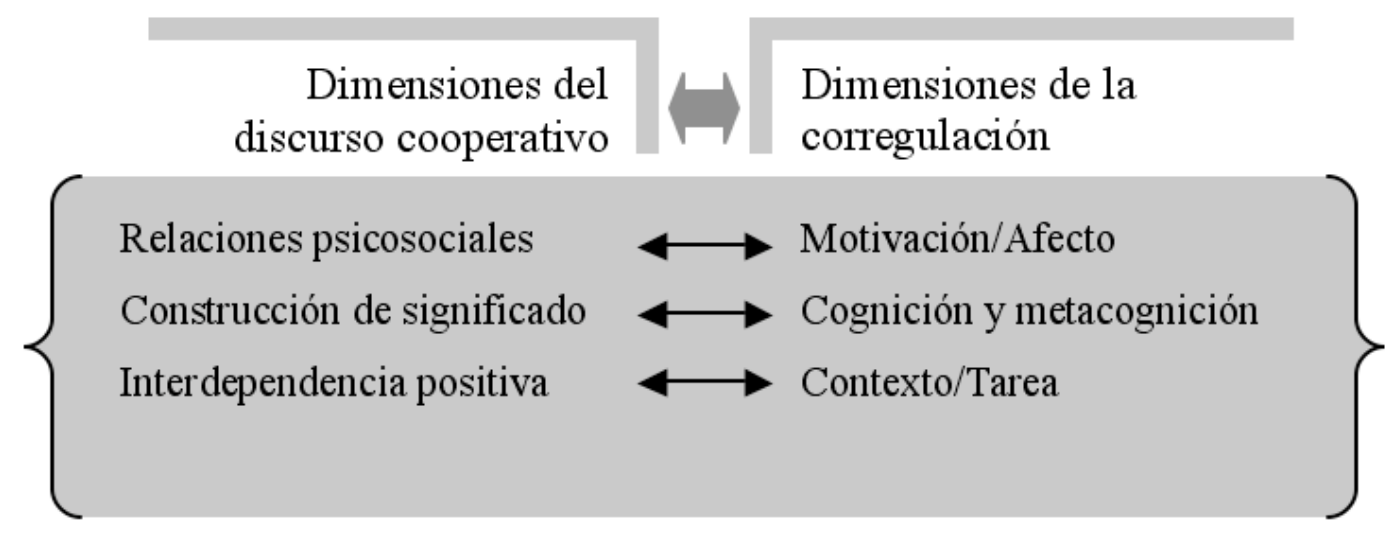

Fig. 1 Correspondencia conceptual entre la dimensiones de análisis del discurso cooperativo y de la corregulación.

En este planteamiento es central la idea de que los individuos actúan en contextos sociales (Paris y Paris, 2001; Patrick y Middleton, 2002; Zimmerman, 1995). La autorregulación supone así, el aspecto social que incluye interacción con los pares y profesores que corregulan la manera como el estudiante aprende (Meyer y Turner, 2001).

La corregulación, como forma que adquiere la regulación en el aprendizaje cooperativo, pone énfasis en la apropiación gradual de problemas y tareas comunes, compartidos a través de la interacción interpersonal (Hadwin, Wisney y Ponting, 2005; McCaslin y Hickey, 2001). En este sentido el término "regulación compartida" surge como el modo más efectivo de corregulación (Volet et al., 2008) y se refiere a múltiples individuos que constantemente monitorean y regulan la actividad conjunta (Vauras et al., 2003).

Estas cuestiones, que pueden considerarse básicas para la comprensión de la regulación del aprendizaje, a la hora de estudiarlas en profundidad, deben relacionarse con las características de las tareas y de los contextos en los que transcurre.

Por este motivo para esta investigación se amplía la revisión teórica más allá del concepto y las bases de la cooperación, con el propósito de encontrar un modelo específico de apropiación del razonamiento estrategicos durante debates en grupo. Las referencias que aportaron mayor información se circunscriben a las investigaciones de Angeli, Valanides y Bonk (2003), Campos (2004), Garrison et al., (2000), Hara, Bonk y Angeli (2000), Murphy 
(2004), Wang, Sierra y Folger (2003), Weinberger y Fischer (2006) y Zhu (1996). Estas aportaciones tienen en común el análisis de la construcción de conocimiento durante el debate en contextos virtuales de aprendizaje.

Atendiendo al propósito de este estudio, se adopta la propuesta de Weinberger (2006) que proporciona un esquema para analizar múltiples dimensiones que se manifiestan durante la construcción de conocimiento en CSCL. Entre las dimensiones se encuentran la de la participación, la epistémica, la argumentativa y la de los modos sociales de construcción conjunta. Esta última dimensión describe en qué medida los estudiantes se refieren a las contribuciones de sus compañeros durante una tarea cooperativa de aprendizaje, un aspecto que ha sido vinculado a la adquisición de conocimientos (Fischer, Bruhn, Gräsel y Mandl, 2002; Teasley, 1997). Con esta propuesta, los segmentos de discurso pueden ser evaluados con respecto a cómo los alumnos trabajan en la tarea y cómo formulan argumentos desde la cooperación.

Complementariamente se adopta la propuesta de Anderson et al., (2001), que entiende el razonamiento como un proceso de argumentación, lo cual coincide con la demanda cognitiva de la tarea cooperativa que se plantea a los estudiantes que participan en este estudio. Una característica importante de un esquema argumentativo es que es abstracto y, por lo tanto, permite transferencia o generalización entre situaciones. Desde este punto de vista, el razonamiento consiste en un flujo de proposiciones dentro de un discurso de razones argumentadas (Reznitskaya, Kuo, Glina y Anderson, 2008).

Las aportaciones de estos autores se concretan en la descripción de un esquema argumentativo Argument Schema Theory - AST -, que permite visualizar como los participantes en una discusión organizan la información (elaboran argumentos pertinentes) y recuperan esta información para elaborar nuevos argumentos. A su vez este mecanismo proporciona la base para anticipar objeciones y encontrar insuficiencias en los argumentos propios y de los otros que participan en la discusión.

Esta formulación permite a especificar las estrategias de razonamiento como formas particulares de argumentación y ofrece criterios, a nuestro entender, de gran utilidad para su evaluación. Se asume así que el conocimiento emerge del debate en grupo durante tareas cooperativas, es fundamentalmente dialógico y hace referencia a las influencias sociales en el desarrollo del razonamiento. Concretamente se refiere al aprendizaje como un proceso de interiorización, entendido como apropiación participativa, emergente de la interacción social 
(Rogoff, 1995, Vygotsky, 1979; Wertsch, 1991). "Razonar, pensar exige escuchar varias voces, contrastar diferentes perspectivas de un asunto. La capacidad y la disposición para tomar más de una perspectiva surge de tomar parte en discusiones con otros que tienen perspectivas diferentes" (Anderson, et al., 2001, p. 2)

Sobre la base de este marco conceptual y de acuerdo con el carácter práctico de este estudio, la pregunta central de esta investigación es la siguiente: ¿Cuáles indicadores de la corregulación son de interés para la evaluación del aprendizaje en tareas cooperativas con demandas argumentativas en entornos de aprendizaje virtuales asincrónicos y escritos?

Teniendo en cuenta estos presupuestos conceptuales, globalmente, queda enfatizada la idea de que la buena construcción de conocimiento en tareas cooperativas en entornos virtuales asincrónicos y escritos supone la regulación del proceso de aprendizaje. Más exactamente y ligado con el objetivo de este trabajo, se entiende que la calidad de la corregulación da cuenta de la eficacia para la construcción de conocimiento en tareas de aprendizaje colaborativo, entendido como un flujo de razonamientos argumentados con base en el diálogo social.

Sobre estos supuestos y a modo de guía para la aproximación progresiva a la respuesta a la pregunta formulada se establecen dos hipótesis:

PRIMERO: La observación de los mecanismos de la cooperación en un debate virtual, particularmente de los lenguajes a través de los que se manifiestan, permiten apreciar los diversos modos de regulación de la actividad de estudio que realizan los estudiantes (autoregulación, regulación externa y regulación conjunta).

SEGUNDO: El trabajo cooperativo en forma de debate para el estudio de casos en un entorno virtual de comunicación asincrónica y escrita, exige a los estudiantes diversas y particulares estrategias de regulación de la actividad individual y conjunta, para que, en efecto, la discusión y el trabajo cooperativo conduzca a la elaboración de ideas debidamente argumentadas. 


\section{Marco empíririco}

\subsection{Diseño de la investigación}

\subsubsection{Objetivos de la investigación}

La finalidad de la presente investigación es describir modelos de interacción que tienen lugar durante la cooperación, que permitan identificar indicadores para observar y evaluar la regulación del aprendizaje durante tareas cooperativas en entornos de aprendizaje virtuales asincrónicos y escritos.

Para alcanzar el objetivo propuesto se plantea la pregunta ¿Cuáles indicadores de la corregulación son de interés para la evaluación del aprendizaje en tareas cooperativas en entornos de aprendizaje virtuales asincrónicos y escritos?

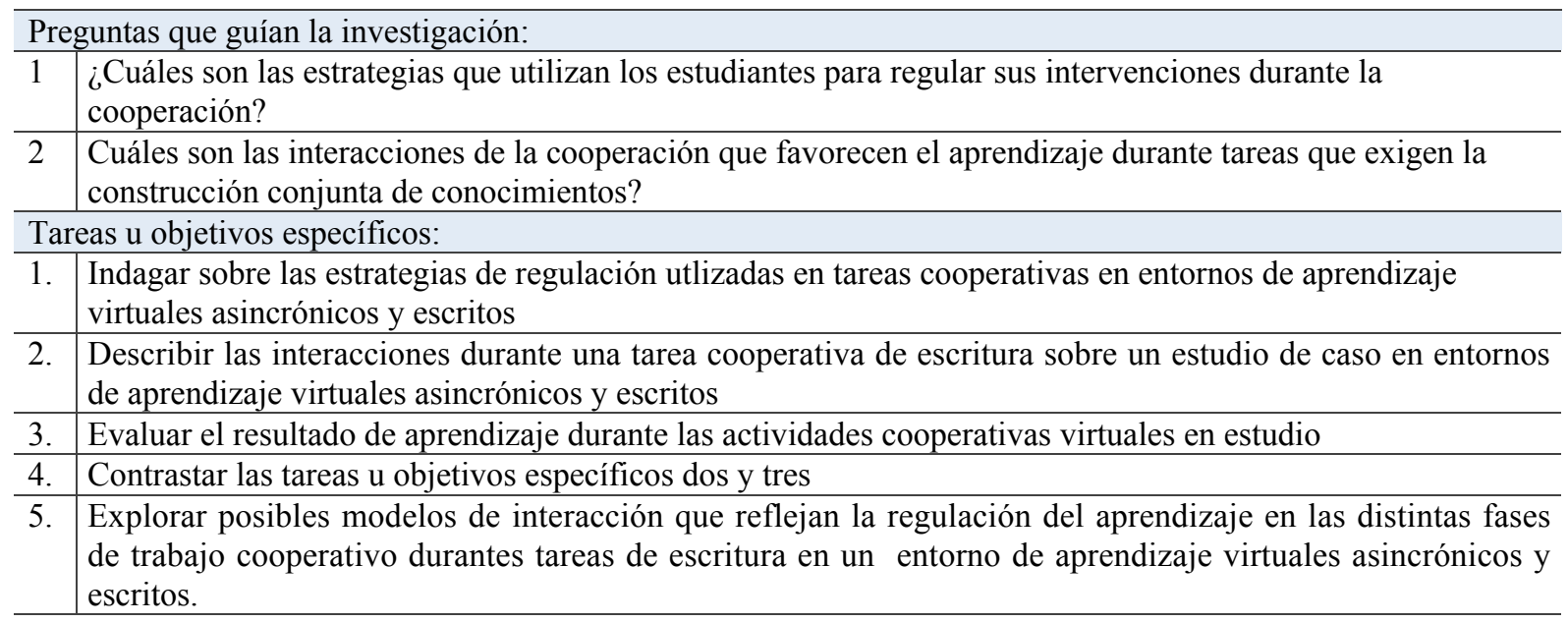

Tabla 1 Preguntas de investigación y tareas u objetivos específicos

\subsubsection{Metodología empleada}

Para el desarrollo de los objetivos de la presente investigación se optó por aplicar una metodología de estudio de caso y por el análisis del discurso de los estudiantes en el proceso de trabajo cooperativo. La investigación se diseñó para llevarse a cabo en cinco fases.

En una primera fase se familiarizó con el entorno virtual en el que se desarrolla la experiencia en estudio, con el tema de la asignatura a analizar, con los materiales y pautas ofrecidas, y con los participantes del estudio, mediante sus propios referentes.

En la siguiente fase, se describieron las interacciones durante una tarea cooperativa de escritura, mediante la observación sistematizada de la cooperación de las actividades objeto de estudio. Para esto fue necesaria la elaboración de un sistema de categorías que permitiera 
explorar e identificar categorías de discurso que reflejaran la presencia de regulación social y regulación cognitiva.

Seguidamente, con la colaboración de la docente, se evaluó el resultado de aprendizaje de los grupos, reflejado en los informes de dos secuencias didácticas. Estos resultados fueron contrastados, enseguida, con las observaciones de los grupos, realizadas en el transcurso de los debates virtuales de la segunda fase.

En una última fase se exploraron posibles modelos de interacción, que permitieran observar la regulación del aprendizaje durante tareas cooperativas en entornos de aprendizaje virtuales asincrónicos y escritos.

En la figura (Fig. 2) se presenta el orden sistemático seguido en esta investigación.

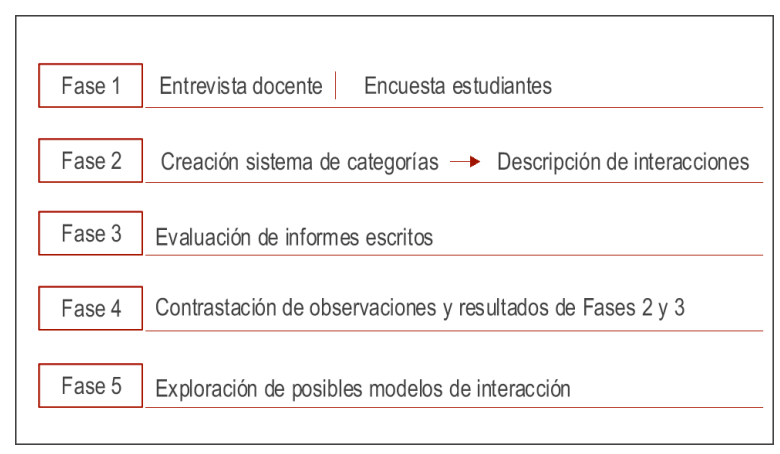

Fig. 2 Procedimiento de la investigación

\subsection{Presentación del caso}

La experiencia se desarrolla en el marco de la asignatura "Planificación de procesos docentes de formación con Tecnologías de la Información y de la Comunicación (TIC)”, de 3 créditos ECTS (European Credit Transfer System), del máster en Educación y TIC (E-learning) de la Universitat Oberta de Catalunya (UOC). El objetivo general de la asignatura es el de desarrollar las competencias profesionales que le permitan a los estudiantes planificar procesos educativos y formativos con el uso de las TIC, para el que se propone el estudio de tres casos reales. El proceso de enseñanza-aprendizaje se realiza a través del aula de campus virtual de la UOC.

A continuación se describen las características del entorno educativo en el cual se enmarca la experiencia, y más adelante se detalla la situación didactica en la que se centra esta investigación. 


\subsubsection{El entorno de aprendizaje de la Universitat Oberta de Catalunya (UOC)}

El modelo educactivo de la Universitat Oberta de Catalunya (UOC) se basa en un entorno virtual de comunicación, al que se conoce como campus virtual (Anexo G). Éste es un espacio de apoyo y de aprendizaje desde donde se posibilita la interacción con la comunidad universitaria y desde el cual se tiene acceso a diversos recursos y servicios, entre los más representativos se encuentran: el Aula virtual, la Biblioteca virtual y los Materiales didácticos de elaboración propia.

El estudiante de la UOC determina y regula su propio proceso de aprendizaje, es el que aporta sus propias estrategias para buscar, encontrar, seleccionar y aplicar lo que necesita para lograr un objetivo fijado. Gracias a las nuevas tecnologías es posible acortar las barreras del tiempo y el espacio, y de este modo el estudiante puede gestionar su propio tiempo, planificar su ritmo de estudio y construir su itinerario académico, para seguir su proceso formativo enlínea.

En el Aula virtual, disponible las 24 horas del día, tiene lugar la interacción, es decir el intercambio de comunicación entre los estudiantes y los docentes en las diferentes asignaturas. Es aquí donde se comparten propuestas, ideas, se negocian significados, a partir de los cuales se puede generar conocimiento. Con este fín, el área del Aula virtual presenta una estructura funcional, ofreciendo los siguientes principales espacios: Comunicación, Planificación, Recursos y Evaluación.

A continuación se describe únicamente el espacio de la comunicación, en el cual se realiza la actividad analizada y en el que se proporcionan herramientas para la construcción de conocimiento.

El espacio para la comunicación (Fig. 2) es exclusivo para la interacción entre los estudiantes y el docente. Esta área se divide en cinco espacios de comunicación, agrupados en un solo sistema de buzones, y son utilizados tanto por los estudiantes como por el cuerpo de personas que compone la coordinación de la asignatura.

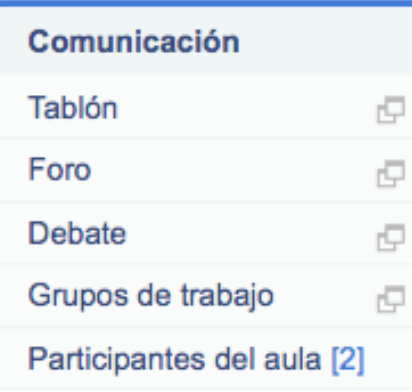

Fig. 3 Espacio para la comunciación de la UOC http://www.uoc.edu/ o el coordinador de la asignatura. Los espacios de foro y debate temáticos son de uso exclusivo de los estudiantes y el docente. Los estudiantes realizan aquí su intercambio académico a través de las pautas ofrecidas por el 
docente, quien organiza, coordina y participa en alguno de los espacios previamente definidos con el fín de cumplir con el objetivo de la actividad y enriquecer así las experiencias de aprendizaje.

El espacio grupos de trabajo, se refiere al espacio de trabajo del que cada grupo dispone para realizar una actividad. Este espacio ofrece privacidad a cada grupo y a él sólo pueden acceder sus miembros y el docente.

El espacio de comunicación también mantiene al estudiante informado del estado en-línea de sus compañeros de asignatura, de manera que pueda tomar contacto inmediato con él o ella ya sea por medio del correo electrónico o por medio del chat.

Los mensajes que han sido enviados a cualquiera de los espacios de comunicación anteriormente mencionados, quedan registrados y pueden ser consultados cuando se requiera. En el espacio de debate (Anexo G), por ejemplo, se ofrece diferentes opciones para los mensajes como lo son: leer, crear un nuevo mensaje, responder mensaje al espacio de debate o responderlo sólo al remitente, reenviar el mensaje, ver el historial del mensaje de quién y cuándo lo ha leido, ordenar e incluso exportarlo. Según la actividad a realizar, y a petición de los alumnos o por iniciativa del docente, es posible crear carpetas de debate, con el ánimo de enfocar las intervenciones y mantener una organización en el transcurso del debate.

\subsubsection{Presentación de la situación didáctica}

Para comprender el contexto de enseñanza y aprendizaje en el que se enmarcan las actividades de estudio, se describen a continuación los aspectos relacionados con la asignatura, el contenido y los materiales, seguido de los participantes y el diseño del curso.

Las secuencias didácticas de aprendizaje cooperativo virtual estudiadas, se desarrollan en el marco de la asignatura de máster en E-learning llamada Planificación de procesos docentes de formación con Tecnologías de la Información y de la Comunicación (TIC).

\subsubsection{Asignatura, contenido y materiales}

Esta asignatura aplica en sus actividades las técnicas de aprendizaje colaborativo de estudio de casos, para la resolución de problemas, y el debate crítico, para estimular el diálogo entre los participantes (Casanova, 2008). El contenido de la asignatura se compone del estudio de tres casos reales, que correponden a tres tipos de experiencias que utilizan las TIC en sus procesos formativos: Caso 1: Experiencia docente de tipo Presencial, Caso 2: Experiencia docente de tipo Virtual, Caso 3: Experiencia docente de tipo Semi-presencial. 
Los materiales del curso son principalmente tres documentos hipermedia referidos a la planificación para la docencia presencial, virtual y semi-presencial con TIC. Además se ofrecen otros recursos como el plan docente y bibliografía recomendada, orientaciones para la lectura comprensiva y la escritura de informes, orientaciones para el desarrollo del debate, pautas para la auto-evaluación y la co-evaluación del proceso, anexos y páginas web para acceder desde el campus virtual.

\subsubsection{Participantes}

a) Los participantes en este estudio son 25 estudiantes y una consultora/docente. Todos los estudiantes poseen titulaciones profesionales y algunos de ellos además cuentan con algún tipo de formación de pos-título o de postgrado. Para la asignatura se requirió la formación de grupos.

Los grupos de trabajo se conformaron a partir de la preferencia de los estudiantes por uno de los casos de estudio propuestos. Estas preferencias se justificaron de acuerdo a la relación del estudiante con el caso en función de sus experiencias, conocimientos, intereses personales y/o profesionales. Con esta misma configuración grupal se desarollaron ambas secuencias didácticas estudiadas.

El resultado de la conformación de los grupos en esta asignatura fue de seis grupos de los cuales, por razones de densidad de material, se analizaron cinco de ellos. En la Tabla 2 se muestra la conformación de los cinco grupos analizados, el número de participantes y el caso en estudio elegido.

Tabla 2

Conformación de grupos

$\begin{array}{ccc}\text { Grupos } & \text { Número de Participantes } & \text { Caso elegido } \\ \text { A } & 5 & \text { Modelo semi-presencial (Caso 3). } \\ \text { B } & 4 & \text { Modelo semi-presencial (Caso 3). } \\ \text { C } & 4 & \text { Modelo virtual (Caso 2) } \\ \text { D } & 6 & \text { Modelo virtual (Caso 2) } \\ \text { E } & 6 & \text { Modelo presencial (Caso 1) }\end{array}$

Los grupos dispusieron de un propio espacio de trabajo dentro del área de debate en el aula vitual para llevar a cabo las discusiones críticas, recibieron las mismas instrucciones de la docente y contaron con el mismo periodo de tiempo para el desarrollo de las actividades.

b) La docente impartió las instrucciones sobre la actividad a realizar a través de dos mensajes al tablón, previos al comienzo de cada actividad. En los mensajes también sugirió pautas para poner en práctica con el fín de que el trabajo cooperativo resultara provechoso para todos los participantes, procurando que se hicieran intervenciones orientadas hacia la construcción conjunta de conocimiento. 
El rol de la docente en estas actividades fue principalmente de guía y orientación, prestándose atenta a cualquier inquietud mediante su correo electrónico. Su función además, fue la de intervenir en situaciones donde requirieron la regulación del intercambio, ya sea con sugerencias y/o aclaraciones sobre la tarea o con refuerzos sociales. Una vez terminada la actividad, la docente intervino para dar acuse de recibo de la tarea, comentar el desarrollo del debate, felicitar a los grupos por su esfuerzo y ofreció un análisis grupal y algunas veces individual, si lo considerara necesario, con respecto a la calidad de las contribuciones de cada integrante hacia el grupo en función de la pauta previamente entregada.

\subsubsection{Diseño del curso}

El curso está constituido por tres actividades planeadas para un semestre y tienen un diseño estratégico orientado a la sistematización del conocimiento y a impulsar las estrategias para el aprendizaje cooperativo en entornos virtuales (Alvarez y Guasch, 2006).

Para alcanzar los objetivos propuestos y dar respuesta a las preguntas de investigación formuladas, este estudio se circunscribe a las actividades 2 "Debate en pequeños grupos de discusión cooperativa" y 3 "Debate constructivo". En estas actividades se desarrolla un proceso de aprendizaje cooperativo en grupos que aplican la técnica de estudio de caso en un contexto virtual. Cada actividad constituye una secuencia didáctica, al poseer un objetivo, un desarrollo de la actividad, en nuestro caso el debate, y una evaluación.

La demanda cognitiva de las tareas es básicamente argumentativa con soporte en la discusión colectiva, que se lleva a cabo mediante debates críticos virtuales y se concreta a través de un informe escrito para cada actividad.

Los criterios de evaluación de las actividades a analizar, se enfocan tanto a la calidad del debate, la cooperación grupal e individual, así como a la calidad de la construcción del conocimiento, esto es, la argumentación en el análisis crítico del caso, reflejado no sólo en las discusiones generados a través del debate, sino en los informes escritos. Esta asignatura se caracteriza por aplicar la co-evaluación como técnica evaluativa. De esta manera se estimula el diálogo entre docente y estudiantes en un espacio virtual común para evaluar su propio producto. Para apoyar el proceso de co-evaluación, la docente introdujo la herramienta de anotaciones basada en la red llamada annotation tool $^{l}$, y habilitó espacios para cada grupo. En la siguiente tabla se ofrece un resumen del diseño del curso.

\footnotetext{
${ }^{1}$ http://www.annotatiesysteem.nl/
} 


\begin{tabular}{|c|c|c|c|}
\hline \multirow{2}{*}{$\begin{array}{l}\text { Actividad y } \\
\text { temporalización }\end{array}$} & Actividad 1: & Actividad 2 & Actividad 3: \\
\hline & $\begin{array}{l}\text { Resumen contrastado } \\
\text { Dos semanas }\end{array}$ & $\begin{array}{l}\text { Debate en pequeños grupos } \\
\text { de discusión } \\
\text { Cuatro semanas }\end{array}$ & $\begin{array}{l}\text { Debate constructivo } \\
\text { Cuatro semanas }\end{array}$ \\
\hline $\begin{array}{l}\text { Objetivo de la } \\
\text { actividad }\end{array}$ & $\begin{array}{l}\text { Identificar las aciones que } \\
\text { distinguen la estrategia de } \\
\text { planificación del proceso } \\
\text { docente en tres casos } \\
\text { diferentes de instituciones } \\
\text { educativas que introducen } \\
\text { las TIC. }\end{array}$ & $\begin{array}{l}\text { Valorar críticamente la } \\
\text { estrategia para la } \\
\text { introducción de las TIC en } \\
\text { cada una de las experiencias } \\
\text { educativas expuestas al } \\
\text { debate. }\end{array}$ & $\begin{array}{l}\text { Intervenir crítica y } \\
\text { creativamente en el análisis y } \\
\text { desarrollo de propuestas } \\
\text { docentes e-learning para la } \\
\text { formación profesional. }\end{array}$ \\
\hline $\begin{array}{l}\text { Productos y } \\
\text { modalidad } \\
\text { (individual o } \\
\text { grupal) }\end{array}$ & $\begin{array}{l}\text { a) Elaborar un cuadro } \\
\text { resumen comparativo de los } \\
\text { tres casos de tipo de } \\
\text { planificación con TIC } \\
\text { (lectura y escritura } \\
\text { individual) } \\
\text { b) Compartir/contrastar el } \\
\text { resumen elaborado (en } \\
\text { grupos) }\end{array}$ & $\begin{array}{l}\text { a) Debatir en torno a la } \\
\text { pregunta ¿cómo cambia el } \\
\text { proceso de enseñanza y } \\
\text { aprendizaje con la } \\
\text { introducción de las TIC? } \\
\text { (discusión grupal) } \\
\text { b) Elaborar } \\
\text { cooperativamente un } \\
\text { informe final donde se } \\
\text { expresen los logros, } \\
\text { dificultades, riesgos y retos } \\
\text { del proceso de enseñanza y } \\
\text { aprendizaje con la } \\
\text { introducción de las TIC } \\
\text { (elaboración grupal) } \\
\text { c) Co-evaluar el producto } \\
\text { para su optimización y } \\
\text { posterior entrega }\end{array}$ & $\begin{array}{l}\text { a) Compartir/contrastar el } \\
\text { informe elaborado en la } \\
\text { actividad anterior (grupo } \\
\text { clase) } \\
\text { b) Revisar y comentar el } \\
\text { informe de sus compañeros }\end{array}$ \\
\hline $\begin{array}{c}\text { Recursos y } \\
\text { herramientas }\end{array}$ & $\begin{array}{l}\text { Documentos de } \\
\text { planificación para la } \\
\text { docencia en los tres casos } \\
\text { Pauta para la lectura } \\
\text { comprensiva y la escritura } \\
\text { de texto en formato } \\
\text { resumen } \\
\text { Pautas para la } \\
\text { autoevaluación } \\
\text { Pauta para la co-evaluación }\end{array}$ & $\begin{array}{l}\text { Documentos de } \\
\text { planificación para la } \\
\text { docencia en los tres casos } \\
\text { Pauta para la co-evaluación } \\
\text { Herramienta para la } \\
\text { evaluación en línea } \\
\text { annotation tool }\end{array}$ & $\begin{array}{l}\text { Documentos de planificación } \\
\text { para la docencia en los tres } \\
\text { casos } \\
\text { Pautas para la co-evaluación } \\
\text { Pauta para la autoevaluación } \\
\text { final }\end{array}$ \\
\hline
\end{tabular}

Tabla 3 Resumen del Diseño del curso

Para el desarrollo de ambas actividades, los estudiantes reciben instrucciones de la docente referidas a las estrategias para la realización del debate y para la construcción de los argumentos, enfatizando en que las intervenciones tengan en cuenta los indicadores básicos y generales de la calidad de aprendizaje y que se orienten a propósitos como:

- Compartir y comparar información

- Definir, describir o identificar tópicos de interés o de problema

- Identificar y explorar la disonancia o inconsistencia de ideas, conceptos o enunciados

- Negociar significados, co-construir conocimiento, ofrecer argumentos, contra argumentos, evidencias, etc. 
- Modificar enunciados iniciales, proponer integración y síntesis

- Concretar acuerdos y conocimientos construidos

- Compartir reflexiones metacognitivas que ilustren el entendimiento de que el conocimiento o formas de pensamiento (esquema cognitivo) ha cambiado como resultado de la interacción en las situaciones de aprendizaje

\subsection{Planificación e implementación del trabajo de campo}

La investigación se desarrolló siguiendo el siguiente cronograma.

\begin{tabular}{|l|l|l|l|l|}
\hline No & Fases & Fecha Inicio & Fecha final & Tiempo/ Semanas \\
\hline 1 & $\begin{array}{l}\text { Planteamiento del Problema, } \\
\text { Objetivo y preguntas de } \\
\text { investigación }\end{array}$ & 11-Febrero-09 & 3-Marzo-09 & 3 semanas \\
\hline 2 & Marco Conceptual & 4-Marzo-09 & 8-Abril-09 & 5 semanas \\
\hline 3 & Marco Empírico & 4-Marzo-09 & 20-Mayo-09 & 11 semanas \\
\hline 4 & $\begin{array}{l}\text { Resultados, Discusión y } \\
\text { Conclusiones }\end{array}$ & 21-Mayo-09 & 31-Mayo-09 & 2 semanas \\
\hline
\end{tabular}

Tabla 4 Cronograma de actividades a realizar

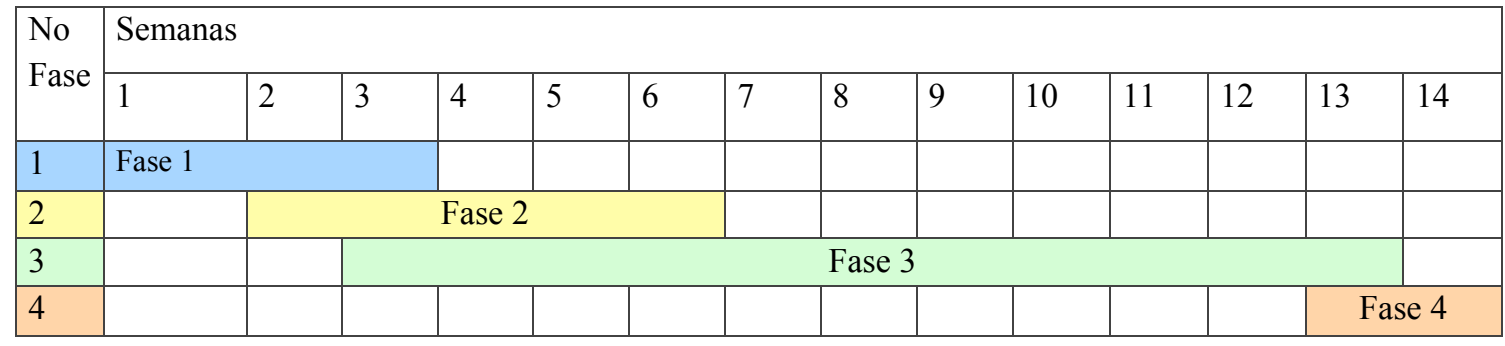

Tabla 5 Cronograma de actividades a realizar por semana

\subsection{Valoración y discusión de la implementación de la planificación}

El cronograma inicial presentado en el anexo A, se siguió con muy pocas modificaciones. La comunicación con la supervisora de la investigación fue bastante frecuente y provechosa, esto influyó positivamente en el desarrollo del proyecto y en la motivación de la investigadora. A continuación se presenta el registro de las interacciones.

\begin{tabular}{|l|c|l|l|}
\hline \multicolumn{3}{|l|}{ Registro de comunicación entre Investigadora y Supervisora } \\
\hline E-mails intercambiados & 107 & Desde febrero 11 hasta el 31 de Mayo \\
\hline Encuentros virtuales por Skype & 11 & Feb 11 - Marzo 4, 18 - Abril 3, 4 - Mayo 6, 22, 27, 28, 29, 30 \\
\hline Encuentros presenciales & 1 & del 24 al 26 de Abril en Barcelona \\
\hline Otras herramientas & \multicolumn{3}{|l|}{ Google Docs y Annotation tool } \\
\hline
\end{tabular}

Tabla 6 Registro de comunicación 


\subsection{Estrategia analítica}

Para la obtención, elaboración y posterior análisis de los datos, que correspondieran con la metodología de la investigación, se combinaron diferentes técnicas para la recopilación de la información, con el objeto de contrastar los datos desde diversas fuentes.

A través de una entrevista semiestructurada (León y Montero, 2003), se conocieron las referencias de la docente sobre la actividad de estudio, específicamente la concepción de la actividad conjunta (aprendizaje cooperativo en entornos virtuales) y de la evaluación. (Anexo C)

Seguidamente se analizaron cualitativamente 12 encuestas en línea (Anexo D), rellenadas voluntariamente por los estudiantes, al comienzo de las actividades de la asignatura. Estas encuestas fueron llevadas a cabo por un grupo investigativo de la UOC, del que la docente de la asignatura en estudio forma parte. Con la encuesta se indagó sobre las experiencias de aprendizaje cooperativo en entornos virtuales de aprendizaje, particularmente se preguntó sobre las estrategias de regulación que habitualmente utilizaban durante la realización de tareas cooperativas que requerían la escritura, como lo es un informe de la discusión de un caso en estudio; los obstáculos que enfrentan para la elaboración de textos como tarea de aprendizaje en línea y las estrategias del docente que se consideraban útiles para la realización efectiva de la actividad.

Para describir las interacciones durante las tareas cooperativas de escritura, se adoptaron como secuencias didácticas las actividades 2 "Debate en pequeños grupos de discusión cooperativa" y 3 "Debate constructivo". Estas actividades contemplan en su diseño un objetivo, una tarea y una evaluación y se infiere que los debates generados se conformaron por un inicio, un desarrollo y un final.

Se recuperó la memoria de los debates de las dos secuencias didácticas. Los datos se prepararon editando todos los mensajes registrados en el aula, mensajes al tablón, debate y al foro. De los cinco grupos estudiados se prepararon los siguientes datos:

- Corpus con el texto de los mensajes del debate para la segunda actividad de estudio

- Corpus con el texto de los mensajes del debate para la tercera actividad de estudio

La preparación de ambos corpus, en unidades de significado, pensamiento o ideas denominadas unidades temáticas (UT), fue riguroso y cuidadoso. El análisis se realizó con el programa científico exclusivo para análisis cualitativo Atlas.ti Versión 6. 
Las intervenciones de los debates se organizaron por fases de trabajo cooperativo (Casanova, 2008; Gunawardena, Lowe y Anderson, 1997) y además por hilos de discusión, lo cual favoreció para conocer a fondo cada secuencia de interacción e identificar las funciones, intenciones y el alcance de las expresiones en cada fase.

El resultado del número total de unidades temáticas analizado en ambas actividades, al igual que el número de documentos intercambiados durante ambas actividades en cada grupo, se puede observar en la tabla 7. En la primera secuencia didáctica se contó con un total de 905 UT, donde el grupo A presentó 164 UT, el grupo B 124 UT, el grupo C 211 UT, el grupo D 152 UT y el grupo E 254 UT. En la segunda secuencia didáctica, el número de unidates temáticas disminuyó a 529 UT, el grupo A presentó 125 UT, el grupo B 89 UT, el grupo C 79 UT, el grupo D 81 UT y el grupo E 155 UT. El número total de documentos intercambiados durante ambas secuencias fue de 135, el grupo A intercambió 18 documentos, el B 27, el C 7, el D 19 y el E 64.

\begin{tabular}{ccccc}
\hline & $\begin{array}{c}\text { Número de } \\
\text { Participantes }\end{array}$ & Unidades temáticas (UT) & $\begin{array}{c}\text { Número de } \\
\text { documentos }\end{array}$ \\
Grupos & & Actividad 2 & Actividad 3 & intercambiados \\
A & 5 & 164 & 125 & 18 \\
B & 4 & 124 & 89 & 27 \\
C & 4 & 211 & 79 & 7 \\
D & 6 & 152 & 81 & 19 \\
E & 6 & 254 & 155 & 64 \\
Totales & 25 & 905 & 529 & 135 \\
& & \multicolumn{2}{c}{ Total: 1434 UT } \\
\end{tabular}

Tabla 7 Unidades temáticas analizadas en la Actividad 2 y 3

- Documentos intercambiados durante los debates

Atendiendo a las diversas estrategias de cooperación empleadas por los grupos, es importante hacer referencia a que los documentos intercambiados durante la cooperación dentro del espacio virtual de la UOC fueron recopilados y analizados para la investigación. (ver tabla 7)

- Mensajes enviados por la docente al tablón del aula, relativos a las actividades de estudio de interés y a la evaluación propiamente.

Estos mensajes sirvieron para conocer la forma en la que la docente orientó a los estudiantes durante el transcurso de las actividades en la asignatura.

Los resultados del aprendizaje de los grupos, se evaluaron mediante:

- Informes de la tarea, el texto elaborado con base en la discusión del caso. 
Con el objeto de conocer el resultado de aprendizaje y cooperación conjunta reflejadas en los informes de ambas secuencias didácticas y poderlos contrastar con las observaciones realizadas en el transcurso de los debates virtuales, se requirió de la colaboración de la docente, quien compartió los resultados de su análisis para la investigación. La docente análizó un total de 10 informes escritos, uno por cada grupo en cada actividad. Los datos acerca de la extensión de los documentos en palabras, fueron proporcionados por la docente y se presentan en la tabla 8 .

\begin{tabular}{|l|l|l|l|l|l|}
\hline Total palabras & \multicolumn{1}{|c|}{ G-A } & \multicolumn{1}{c|}{ G-B } & \multicolumn{1}{c|}{ G-C } & \multicolumn{1}{c|}{ G-D } & \multicolumn{1}{c|}{ G-E } \\
\hline Texto - Act 2 & 6,533 & 5,836 & 2,901 & 4,856 & 4,386 \\
\hline Texto - Act 3 & 6,877 & 9,214 & 1,650 & 14,4004 & 5,800 \\
\hline
\end{tabular}

Tabla 8 Aspectos formales del texto

Nota: La tabla muestra la cantidad de palabras utilizadas en los informes escritos de cada grupo en las dos actividades en estudio. G- Grupo.

\subsection{El Análisis del Discurso: procedimiento de apoyo a la evaluación del debate}

El discurso puede ser considerado como un conjunto de material lingüístico que es coherente en cuanto a organización y contenido y que le permite a las personas construir significados en contextos sociales. El análisis del discurso, se basa en el concepto vygotskiano de la lengua como instrumento sicológico cultural, según proponen y desarrollan investigadores de la perspectiva sociocultural (Casanova, 2008). Los sistemas de símbolos son los recursos que los seres humanos utilizamos para regular los procesos mentales y nuestras acciones, al igual que para regular los procesos mentales y acciones de las personas con las que nos comunicamos e interactuamos.

Cada recurso semiótico poseé sus propias reglas internas de composición y una propia estructura, de modo que el uso de uno u otro subordina por igual la manera como se representa y se transmite la información y los procesos mentales implicados en estas actividades. En función de la naturaleza de la información que se quiera transmitir, de los medios físicos o virtuales de los que se disponga y de los objetivos que se quieran alcanzar con su emisión, se optará ya sea por el uso de uno o por la combinación de algunos. Los recursos semióticos ejercen la labor de mediación de los procesos individuales o intramediales del alumno y de los procesos comunciativos y sociales o intermentales que se ven implicados en el aprendizaje intencional (Coll, 2003).

Con el empleo del análisis del discurso en esta investigación, se pensó que se facilitaría comprender cómo el lenguaje se utiliza para pensar de manera colectiva y además 
comprender la importancia de la calidad de diálogo en los procesos de enseñanza y aprendizaje.

Una vez consensuada la base metodológica para el estudio empírico se realizaron tres niveles de análisis de discurso que se describen a continuación.

\subsubsection{Primer nivel de análisis: Descripción de las interacciones durante tareas cooperativas en entornos asincrónicos virtuales y escritos}

El primer análisis se centró en describir las interacciones de cinco grupos de estudiantes durante las tareas cooperativas, identificando los tipos de habla que reflejaran la presencia de regulación social y cognitiva, así como los tipos de interacciones y los modelos de interacción Para poder observar la regulación en los discursos de los grupos durante la cooperación, fue necesario elegir una unidad básica de categorización que respondiera a los objetivos y a la naturaleza de la discusión cooperativa virtual, y disponer de un sistema de categorías que diferenciara la regulación social de la regulación cognitiva.

Como unidad básica de categorización se eligió la Unidad Temática (UT), entendida como una unidad de significado, introducida por Henri (1992). La unidad temática se refiere a un fragmento de actividad conjunta, es decir segmentos de interactividad, que presentan una determinada estructura de participación y que mantiene una unidad.

Con la unidad temática como base para la categorización, se procedió a la creación de un sistema de categorías apoyado en la teoría y emergente de los datos mismos. El proceso de creación se explica detalladamente en el apartado 3.7. Procedimiento para el análisis de datos.

El análisis de las unidades temáticas en los discursos fue realizado con el programa de análisis cualitativo de datos textuales Atlas.ti Versión 6.

El sistema de categorías fue revisado, evaluado y debatido por parte de tres evaluadores externos. Cada evaluador aplicó las categorías a una gran parte de la secuencia de debate de uno de los cinco grupos en estudio.

Para describir las interacciones durante las tareas cooperativas, se tomaron los mensajes intercambiados durante los debates de los cinco grupos, y en éstos se distinguieron las cuatro fases de trabajo cooperativo: inicio, intercambio, negociación y aplicación (Casanova, 2008; Gunawardena et al., 1997) que permitieron observar mejor el desarrollo de las interacciones en los grupos en el entorno de aprendizaje virtual asincrónico y escrito en estudio. 


\subsubsection{Segundo nivel de análisis: Contrastación del proceso de cooperación con los resultados del aprendizaje}

Este segundo nivel de análisis se concentra en la observación de la evolución de los grupos durante las actividades en estudio. Se consideró que la experiencia cooperativa de la segunda actividad y otros factores, pudieron haber influenciado en las estrategias de interacción reguladoras empleadas en la actividad posterior, centrándose más hacia la construcción del conocimiento, de modo que quedaran reflejas en los informes escritos.

Para analizar y evaluar los informes escritos de cada grupo, se solicitó la colaboración de la docente, quién para este fin adaptó el aporte de Reznitskaya et al. (2008) sobre categorías para evaluar el esquema argumentativo del texto escrito en tareas cooperativas. Los informes textuales fueron codificados con el programa científico para el análisis cualitativo de textos Nvivo QSR Versión 8.

Una vez conocidos los resultados de los análisis por parte de la docente, se retomaron las observaciones realizadas en el primer nivel de análisis y se contrastaron ambos resultados.

\subsubsection{Tercer nivel de análisis: modelos de regulación durante tareas cooperativas virtuales}

Una vez concluidos los dos niveles de análisis anteriores, se procedió a la exploración de modelos de interacción durante las tareas cooperativas estudiadas. Con la identificación de los modelos, se identificaron indicadores para poder observar y evaluar la regulación del aprendizaje.

Para este análisis se tomaron las interacciones del primer análisis, en las que se habían identificado para cada grupo, las cuatro fases de trabajo cooperativo. Éstas permitieron observar la evolución de la construcción del significado conjunto específicamente en entornos de aprendizaje virtuales. La tercera fase de trabajo coopertivo (negociación) se consideró con mayor atención por tratarse de una fase en la que se afirma ocurre la integración de los aportes de las fases anteriores, en donde el conocimiento se justifica de manera más abierta y el razonamiento se visualiza en el discurso, haciendo uso de un lenguaje exploratorio (Mercer, 2004).

Seguidamente se procedió a agrupar los modelos de interacciones encontrados, orientado por la propuesta de Volet et al., (2008) que es un marco de trabajo teórico desarrollado para identificar instancias co-reguladoras de alto nivel de productividad, el cual combina los 
constructos de regulación social y de procesamiento de contenido y los presenta en un sistema de coordenadas con cuatro cuadrantes.

\subsection{Procedimiento para el análisis de datos}

Para analizar las interacciones manifestadas a través del discurso de los estudiantes durante las tareas cooperativas, fue necesaria la creación de un sistema de categorías enfocado hacia la identificación de unidades temáticas que describieran la regulación en los tres modos de regulación: Auto-, externa, y conjunta, en los que se se afirma oscila la verdadera corregulación (Vauras et al., 2003).

La construcción del sistema de categorías resultó de la combinación de un análisis deductivo y uno inductivo. El primero tomó como apoyo las referencias de literatura e investigaciones, que se retoman más adelante en la descripción del proceso, tanto en educación presencial como virtual, que hayan contribuido con las definiciones de categorías de discurso cooperativo; categorías, modelos y características de la regulación del comportamiento tanto individuales como en grupo, en tareas cooperativas y en tareas argumentativas escritas.

El análisis inductivo fue el resultado de lo hallado durante el análisis de los datos. Este complejo proceso de categorización, implicó la re-definición, combinación, ajuste, exclusión o precisión de las categorías hasta lograr un sistema uniforme.

De esta manera, el proceso de categorización se siguió de la siguiente forma:

- Se creó un primer sistema de categorías basado en la teoría y en investigaciones tanto para la regulación social en los tres modos de regulación: auto-, externa y conjunta como para la regulación cognitiva. Las referencias utilizadas en esta primera parte fueron los aportes de Casanova (2008); Vauras et al., (2003) y Volet et al., (2008).

- Con este primer sistema se procedió a codificar una de las actividades de uno de los grupos de estudio en el programa Atlas.ti Versión 6.

- En el proceso de codificación, se encontraron unidades temáticas con características no consideradas en el primer sistema de categorías, por lo que se procedió a describirlas para luego poder agruparlas en nuevas categorias apoyados de las referencias de Biggs y Collis (1982); Bodrova (2006); Green (2001); Järvela et al., (2008); Martin y McLellan (2008). 
- Con lo anterior, se concluyó re-organizar y ajustar las categorías y descriptores de la regulación social, para lo que se consultaron las referencias de Pintrich (2000); Schunk y Zimmerman (1994); Vygotsky (1979); Whipp y Chiarelli (2004); Zimmerman $(1995,1997)$ y de re-definir las de la regulación cognitiva atendiendo a la calidad del procesamiento cognitivo en niveles de procesamiento de contenido: alto $y$ bajo con las referencias de Belland, et al (2008); Biggs (1995); Reznitskaya et al., (2008); Siew y Allison (2005); Volet et al., (2008), para probarlas nuevamente sobre los casos de estudio.

- En este análisis se comprobó que las categorías de regulación social eran suficientemente excluyentes por lo que se procedió a ejemplificar cada categoría con diferentes unidades temáticas del caso en estudio.

En el caso de la regulación cognitiva, se encontraron características en las unidades temáticas que no correspondían a las categorías establecidas y que además indicaban moverse entre los tres modos de regulación: auto-, externa y conjunta, por lo que se continuó con la búsqueda y lectura de referencias que aportaran información sobre el análisis de la construcción de conocimiento durante el debate, encontrándose las siguientes referencias: Angeli et al., (2003), Campos (2004), Garrison et al., (2000) Hara et al., (2000), Murphy (2004), Wang, Sierra y Folger, (2003), Weinberger y Fischer (2006) y Zhu (1996).

- La revisión de las referencias anteriormente mencionadas condujeron a adoptar la propuesta de Weinberger (2006), en especial una parte de su esquema con el que se puede analizar la dimensión social manifiestada durante la construcción de conocimiento en estos entornos en estudio. Es decir, la medida en la que los estudiantes se refieren a las contribuciones de sus compañeros durante una tarea cooperativa de aprendizaje, considerado un aspecto importante en la adquisición de conocimientos. El análisis de la calidad de la argumentación se reforzó con el aporte de Reznitskaya et al., (2008) en tareas escritas.

- Con las nuevas categorías de regulación cognitiva se procedió a codificar nuevamente secuencias dialógicas en la fase de intercambio y negociación de trabajo cooperativo, en donde se afirma encontrar lenguajes relacionados con la construcción de conocimiento (Casanova, 2008), con unos resultados satisfactorios. 
- Seguidamente se ejemplificó cada categoría para poderla integrar adecuadamente en el sistema de categorías.

- El equipo de investigación compuesto por dos investigadoras, se reunió frecuentemente en este proceso, tanto de manera virtual como presencial, para aplicar el sistema de categorías y perfeccionar las definiciones.

- A cada categoría se le asignó un código para agilizar el proceso de categorización por los evaluadores externos, para la gestión de los datos y el análisis de los resultados.

Este laborioso proceso finalizó con la obtención de un sistema de categorías para el análisis de la regulación social y cognitiva en tareas cooperativas en entornos de aprendizaje virtuales asincrónicos y escritos, que se presenta en el apartado 4. Resultados y Discusión.

\subsubsection{Proceso de codificación y de análisis de concordancia entre codificadores.}

El análisis cualitativo que supone esta investigación conllevó a un previo y laborioso proceso de definición y discusión de las categorías, lo cual se realizó en este caso acudiendo al procedimiento de inter-jueces (Patton, 1990).

Con el sistema de categorías construido en el primer análisis, se procedió a seleccionar alguna de las cinco secuencias analizadas para ser evaluada por dos jueces externos. La secuencia seleccionada correspondió al grupo B compuesto por 4 estudiantes que analizaron el estudio de caso 3: Modelo semi-presencial y del que se identificaron 124 unidades temáticas.

La secuencia se organizó por fases de evolución en la construcción de conocimiento (Casanova, 2008; Gunawardena et al., 1997) y se redujo a un 55\% de la extensión original, quedando un total de 69 unidades temáticas para codificar. La reducción de la densidad de las unidades temáticas a evaluar, fue consensuada con uno de los evaluadores, que fue la docente de la asignatura quien estaba ya familiarizada con los casos en estudio. Con los mensajes resultantes, se dispuso de una buena proporción para aplicar las diferentes categorias.

Para la realización de éste proceso, se siguieron los siguientes pasos:

- Búsqueda y elección de dos evaluadores externos más, que así como la docente de la asignatura, fueran profesores de enseñanza universitaria, que contaran con experiencia en investigación cualitativa y que estén familiarizados con la aplicación del aprendizaje cooperativo en entornos virtuales y presenciales. 
- Entrenamiento de los evaluadores externos en el sistema de categorías. En este proceso se debatieron ideas acerca de las categorías menos claras. A partir de esto se precisaron algunas definiciones y se buscaron ejemplos más adecuados.

- La segmentación de las unidades temáticas fue consensuada entre todos, acordando la codificación de 69 unidades temáticas.

- Una vez consensuado lo anterior, cada evaluador externo prosiguió a aplicar las definiciones de las categorías y a codificar las unidades temáticas.

Es necesario indicar que en la primera evaluación hubo conflicto con las categorías de regulación cognitiva, por lo que se decidió discutirlas hasta llegar a un acuerdo final, que es el que se presenta en el apartado 4. Resultados y Discusión

El resultado de la concordancia de las categorizaciones entre los evaluadores externos se pueden observar en el anexo E.

\subsection{Análisis crítico de la metodología utilizada}

La metodología empleada en esta investigación se considera correcta y adecuada para lograr los objetivos planteados. Se optó por el estudio de caso de una experiencia tal y como se ha desarrollado en la práctica para poder avanzar en la comprensión y valoración de situaciones reales de aplicación del aprendizaje cooperativo. Por medio de los casos se pueden extraer conclusiones que, además de contribuir desde un punto de vista teório y metodológico, pueden mostrarse útiles para la mejor implementación de la cooperación en el aula virtual.

El análisis de discurso con su análisis cualitativo de datos, dado en unidades temáticas, fue un proceso complejo y que peligró de subjetividad, pero se procuró superarlo mediante una base teórica sólida y una descripción detallada en las categorías, para que fueran excluyentes y exhaustivas. Este proceso se vió facilitado por la implicación total de la supervisora de la investigación sobre el caso en estudio, con lo que los resultados y categorías surgidas pudieron ser contrastadas y discutidas en el momento necesario.

Es necesario aclarar que el procedimiento debió adecuarse sobre la marcha, puesto que lo estipulado en un comienzo no podía completarse en el tiempo con el que se contaba, o lo que se pensó necesario realizar, ya no era posible por el tiempo transcurrido luego de finalizar la asignatura que se investiga. Por esto, por ejemplo, no se pudo completar el análisis del sexto grupo de trabajo o diseñar otras técnicas de recopilación de datos. 
Para la investigación, ya se contaba con los datos de la encuesta inicial en línea realizada a los estudiantes. Durante el análisis cualitativo se encontró con información que no respondía directamente a las preguntas realizadas, por lo que considera necesario que en próximas prácticas, éstas deben realizarse en función específica a lo que se intenta investigar.

Para poder contrastar mejor los datos sobre la regulación en los grupos, posterior a la finalización del curso, se piensa que con entrevistas voluntarias a algunos de los estudiantes, se hubiera podido conocer más a fondo la experiencia realizada en grupo. Además, se pudo haber indagado acerca de las modificaciones en sus estrategias de regulación y la posible causa de esto.

Para compensar la idea de las entrevistas, se pensó en realizar encuestas en línea tratando de averiguar los datos más concretos, pero el tiempo transcurrido luego de la terminación del curso era bastante largo y hubiera podido alterar las impresiones y respuestas de los estudiantes.

Aunque la cantidad de datos de análisis fue abundante y respondió a los objetivos de la investigación, se piensa que para que ésta cobre más validez, se necesitan analizar los trabajos cooperativos de otros grupos en otras asignaturas en el mismo contexto de estudio, en las que también se apliquen actividades con una demanda cognitiva argumentativa y con otros docentes con estilos y técnicas evaluativas diferentes.

\subsection{Aspecto éticos}

Mediante un mensaje al tablón, los estudiantes fueron informados sobre la vinculación de la asignatura en estudio al proyecto de innovación "Título del proyecto: La regulació de l'estudiant en el procés de construcció d'un ocument en un entorn virtual: l'ús de l'annotation tool - Identificador Oficial: IN-PID0824” de la UOC. Con esto se informó que los resultados de la asignatura iban a ser objeto de investigación.

Con este fin, al comienzo del curso se administró una encuesta en línea, que fue respondida de forma voluntaria y anónima a través de NetQuest.

Todos los datos analizados se trabajaron de manera confidencial. Para proteger la identidad de los participantes en la investigación, se recurrió a las denominaciones: "EXX”, para referirse a uno de los integrantes de grupo, por ejemplo para el grupo A, E05, se refiere al quinto integrante de este grupo; y "La profesora", en el caso en el que la docente fuera llamada por su nombre real. 


\section{Resultados y Discusión}

En este apartado se presentan los resultados de los análisis: Descripción de las interacciones durante tareas cooperativas en entornos asincrónicos virtuales y escritos, Contrastación del proceso de cooperación con los resultados del aprendizaje y modelos de regulación durante tareas cooperativas en entornos asincrónicos virtuales y escritos.

\subsection{Análisis 1: Descripción de las interacciones durante tareas cooperativas en entornos asincrónicos virtuales y escritos}

Con el fin de describir las interacciones de los cinco grupos de estudiantes durante las tareas cooperativas, identificar los tipos de habla que reflejaran la presencia de regulación social y cognitiva, así como los tipos de interacciones y los modelos de interacción, se creó un sistema de categorías.

El sistema de categorías emergente de este primer análisis (Fig. 4) distingue dos dimensiones de la regulación: la dimensión social y la dimensión cognitiva. Cada una de las dimensiones oscila entre tres modos de regulación:

- la autoregulación: se aplica a situaciones en donde se evidencia un proceso de acción individual constructiva que, en función de unos objetivos fijados, se gestiona, se monitorea y se controla.

- la regulación externa: se aplica a situaciones en las que surge un desnivel de comprensión de algún segmento particular de la tarea, por parte de los integrantes del grupo. El sujeto que realiza la regulación externa, es el que se siente mejor familiarizado con ese segmento y quién adopta un papel instructivo con el fín de orientar a los demás a comprender el segmento y así equilibrar el nivel de comprensión grupal.

- la regulación conjunta identificada como el modo más eficaz de corregulación, se refiere a la regulación constante y vigilada de la actividad conjunta, en la que varios integrantes del grupo se implican.

A partir de la teoría y desde los datos se identificaron 14 categorías: 9 categorías en la dimensión de la regulación social y 5 en la dimensión de la regulación cognitiva. 


\section{Categorías de Regulación en trabajo cooperativo virtual}

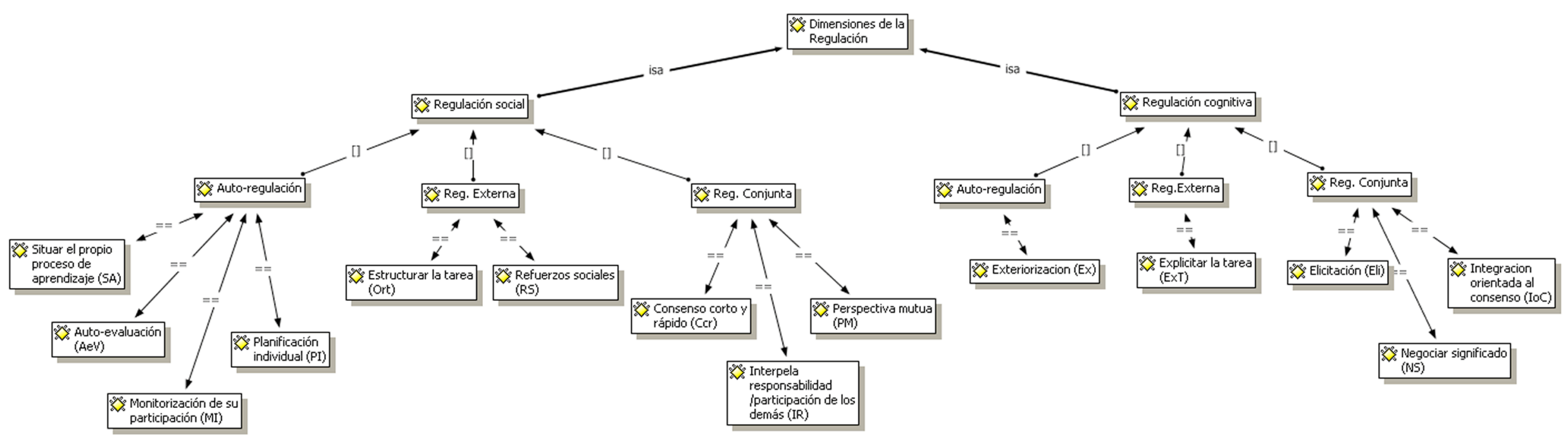

Fig. 4 Categorías de la regulación en las dimensiones social y cognitiva 
Como se puede apreciar en la figura 4, que resume los resultados y relaciones de la categorización realizada con el programa Atlas.ti, se han asociado las categorías de discurso identificadas en el análisis de las secuencias de trabajo de los estudiantes, a dos dimensiones de regulación, que de acuerdo con los postulados expresados en el marco teórico, son esenciales en el aprendizaje cooperativo en entornos virtuales asincrónicos y escritos.

A continuación se describe cada una de las categorías en cada uno de sus modos de regulación y en la dimensión de la regulación correspondiente, en el siguiente orden: primero en la regulación social y luego en la regulación cognitiva. El resumen de las categorías y sus descriptores se puede encontrar en el anexo $\mathrm{H}$.

\subsubsection{Dimensión de la regulación social}

\subsubsection{Autoregulación}

\begin{tabular}{|c|c|}
\hline Auto-evaluación & $\begin{array}{l}\text { Intervenciones que reflejan la valoración de sus conocimientos o experiencias } \\
\text { previas que puedan contribuir a la resolución de la tarea o que reflejen lo que la } \\
\text { resolución de la tarea le aportará respecto a las demandas de su contexto cotidiano. }\end{array}$ \\
\hline Ejemplo: & $\begin{array}{l}\text { "Por mi profesión como orientadora laboral y coordinadora online de cursos } \\
\text { virtuales, me declino por la opción 3. Creo que así podré aprender más cosas que } \\
\text { me sirvan en el día a día de mi jornada laboral" }\end{array}$ \\
\hline $\begin{array}{l}\text { Situar el propio proceso } \\
\text { de aprendizaje }\end{array}$ & $\begin{array}{l}\text { El estudiante asimila el objetivo de la tarea, lo relaciona con sus conocimientos } \\
\text { previos y valora lo que necesita para alcanzarlo. }\end{array}$ \\
\hline Ejemplo: & $\begin{array}{l}\text { "la profundización a hacer está directamente relacionada con la formacion de los } \\
\text { docentes, desde este aspecto y según mi experiencia realizaré el aporte en el docs } \\
\text { creado por E05". }\end{array}$ \\
\hline Planificación individual & $\begin{array}{l}\text { El estudiante valora los recursos y tiempo disponibles para determinar su } \\
\text { colaboración y asume voluntariamente responsabilidades. }\end{array}$ \\
\hline Ejemplo: & $\begin{array}{l}\text { "Deciros que mañana marcho de viaje y no vuelvo hasta el martes, por tanto no me } \\
\text { puedo encargar de elaborar el documento" }\end{array}$ \\
\hline $\begin{array}{l}\text { Monitorización de su } \\
\text { participación }\end{array}$ & Control de la gestión de su propia participación. \\
\hline Ejemplo: & $\begin{array}{l}\text { "A la tarde les mando el documento, con las aportaciones, modificaciones y demás. } \\
\text { Les dije que hoy por la mañana pero quiero hacer unas cosillas más." }\end{array}$ \\
\hline
\end{tabular}

Tabla 9 Categorías dimensión de la regulación social en modo de autoregulación

\subsubsection{Regulación externa}

\begin{tabular}{|c|c|}
\hline Estructurar la tarea & $\begin{array}{l}\text { Intervenciones con preguntas o propuestas sobre organización, procedimiento, } \\
\text { roles, recursos, planificación temporal, formato del texto, etc. }\end{array}$ \\
\hline Ejemplo: & $\begin{array}{l}\text { "Creo que tenemos que fijar una fecha para decidir en qué vamos a centrarnos, y } \\
\text { sugiero el lunes 5." }\end{array}$ \\
\hline Refuerzos sociales & $\begin{array}{l}\text { Intervenciones emotivas que respaldan ideas o actuaciones de otros porque inciden } \\
\text { positivamente en la cognición o en la motivación de los demás. }\end{array}$ \\
\hline Ejemplo: & $\begin{array}{l}\text { „Gracias por el trabajo realizado, en particular a E01 y E02. Sin vuestro tirón } \\
\text { final no lo habríamos conseguido." }\end{array}$ \\
\hline
\end{tabular}

Tabla 10 Categorías dimensión de la regulación social en modo de Regulación externa 


\subsubsection{Regulación conjunta}

\begin{tabular}{l|l}
\hline $\begin{array}{l}\text { Interpelar } \\
\text { responsabilidad/ } \\
\text { participación de los } \\
\text { demás }\end{array}$ & $\begin{array}{l}\text { Interacciones que solicitan la ayuda/colaboración a sus compañeros, en función de } \\
\text { la organización y realización de la tarea. }\end{array}$ \\
\hline Ejemplo: & $\begin{array}{l}\text { "Hay unos comentarios que creo son para E01 y están relacionados con lo que } \\
\text { E03 también menciona respecto al manejo de citas textuales. }\end{array}$ \\
\hline Perspectiva mutua & $\begin{array}{l}\text { Interacciones que comunican un acuerdo recíproco, se reconoce, se valora y se } \\
\text { refuerza una idea. }\end{array}$ \\
\hline Ejemplo: & $\begin{array}{l}\text { "Completamente deacuerdo con E02, la sensación de solo encontrar fortalezas en } \\
\text { el modelo netamente virtual supongo que es porque no somos muy objetivos ya que } \\
\text { estamos siguiendo ese modelo en esta maestria; pero es verdad que los riesgos del } \\
\text { modelo existen [...]" }\end{array}$ \\
\hline $\begin{array}{l}\text { Consenso corto y } \\
\text { rápido }\end{array}$ & Interacciones que muestran conformidad o neutralidad con algo expuesto. \\
\hline Ejemplo: & “Hola! Pues como querais, modelo pedagogico o evaluación... [...]” \\
\hline
\end{tabular}

Tabla 11 Categorías dimensión de la regulación social en modo de Regulación conjunta

\subsubsection{Dimensión de la regulación cognitiva}

\subsubsection{Autoregulación}

\begin{tabular}{l|l}
\hline Exteriorización & $\begin{array}{l}\text { Intervenciones no argumentativas que aportan información de contenido textual o } \\
\text { expresan puntos de vista sobre el contenido a tratar, sin referencias a previas } \\
\text { contribuciones. }\end{array}$ \\
\hline Ejemplo: & $\begin{array}{l}\text { "Desde mi punto de vista creo que Caso 3, cuenta con más fortalezas que } \\
\text { debilidades. A continuación enumero algunas de ellas [...].” }\end{array}$ \\
\hline
\end{tabular}

Tabla 12 Categorías dimensión de la regulación cognitiva en modo de autoregulación

\subsubsection{Regulación externa}

\begin{tabular}{l|l}
\hline Explicitar la tarea & $\begin{array}{l}\text { Interacciones no argumentativas que giran alrededor del objetivo común. El } \\
\text { objetivo de la tarea se analiza, se aclara, se reformula, se repasa. }\end{array}$ \\
\hline \multirow{2}{*}{ Ejemplo: } & $\begin{array}{l}\text { "Estoy deacuerdo con E04 vamos aportando ideas sobre las interrogantes que se } \\
\text {-Conveniencia de la estrategia de planificación seguida, atendiendo a las } \\
\text { necesidades educativas y a las características del contexto donde tiene lugar la } \\
\text { experiencia. } \\
\text {-Fortalezas y debilidades del modelo pedagógico asumido [...]”, }\end{array}$ \\
\hline
\end{tabular}

Tabla 13 Categorías dimensión de la regulación cognitiva en modo de Regulación externa

\subsubsection{Regulación conjunta}

\begin{tabular}{l|l}
\hline Elicitación & $\begin{array}{l}\text { Intervenciones que solicitan, directa o indirectamente, la reacción de otro } \\
\text { compañero(a) en función del contenido de la tarea. }\end{array}$ \\
\hline Ejemplo: & $\begin{array}{l}\text { "Gracias, E01! ¿Creéis que es necesario desarrollar esos cuestionarios o basta } \\
\text { con describir cómo deberían ser?” }\end{array}$ \\
\hline Negociar significado & $\begin{array}{l}\text { Intervenciones argumentadas que ofrecen propuestas, alternativas y complemento a } \\
\text { información intercambiada, con la intención de llegar a un acuerdo. }\end{array}$ \\
\hline
\end{tabular}




\begin{tabular}{l|l}
\hline \multirow{2}{*}{ Ejemplo: } & $\begin{array}{l}\text { "Así que me parece que puede resultar interesante ahondar en las implicaciones y } \\
\text { en los factores de motivación que llevan a una estrategia pensada como apoyo a } \\
\text { procesos presenciales, a terminar con tanta fuerza en el trabajo colaborativo desde } \\
\text { la virtualidad. No solo por el giro en el modelo pedagógico, sino también por las } \\
\text { reflexiones que podemos extraer de nuestra manera de trabajar como equipo a } \\
\text { través de estas herramientas.” }\end{array}$ \\
\hline $\begin{array}{l}\text { Integración orientada } \\
\text { al consenso }\end{array}$ & $\begin{array}{l}\text { Intervenciones argumentadas que manifiestan consenso a partir de integrar } \\
\text { reflexiones propias con la información adquirida de los demás. }\end{array}$ \\
\hline Ejemplo: & $\begin{array}{l}\text { "Respecto al segundo punto [...]” E03 propone [...], yo añadiría que habría que } \\
\text { evaluar, dado que el objetivo último del master era la significatividad laboral de } \\
\text { los conocimientos adquiridos, el número de empresas o personas relacionadas con } \\
\text { el sector empresarial que han participado en la docencia del curso, y preguntarles } \\
\text { a estas personas cómo valoran ese aspecto del master. }\end{array}$ \\
\hline
\end{tabular}

Tabla 14 Categorías dimensión de la regulación cognitiva en modo de Regulación conjunta

\subsection{El comportamiento de la regulación durante las interacciones en el trabajo cooperativo}

Con el sistema de categorías, se analizó el comportamiento de la regulación en las interacciones de todos los grupos durante las actividades en estudio. Es necesario recordar que la demanda cognitiva de ambas actividades era básicamente argumentativa, los grupos dispusieron de un propio espacio de trabajo en el aula virtual para llevar a cabo los debates, todos recibieron las mismas instrucciones de la docente y contaron con el mismo periodo de tiempo para el desarrollo de las actividades: 4 semanas para cada una. Además, en ambas actividades se siguió la misma conformación de los grupos y el producto final a desarrollar fue un informe escrito.

En la siguiente figura (Fig. 5) se presentan los resultados totales de la frecuencia de uso de las 14 categorías de regulación durante las tareas cooperativas. Para este análisis se codificaron 1434 unidades temáticas correspondientes a los discursos de los cinco grupos analizados. Las descripciones de los códigos se pueden apreciar en el anexo $\mathrm{H}$. 
Comportamiento de las categorías de regulación durante las actividades 2 y 3

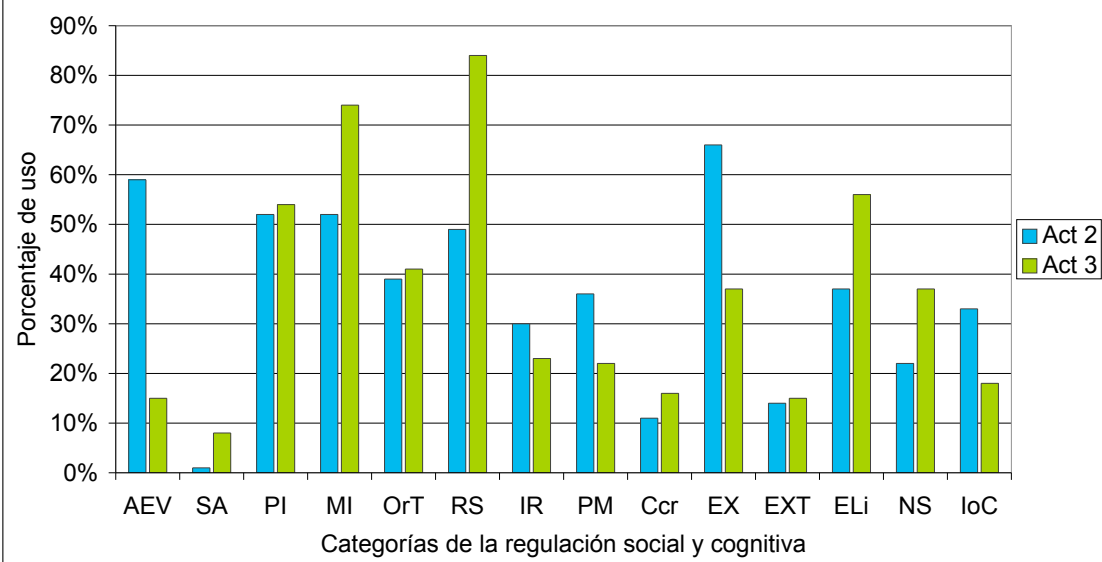

Fig. 5 Comportamiento de las categorías durante las actividades 2 y 3

Para conocer más de cerca la categorización de las interacciones de los grupos, su frecuencia de uso por categoría de regulación en ambas actividades en estudio, se presenta la siguiente tabla.

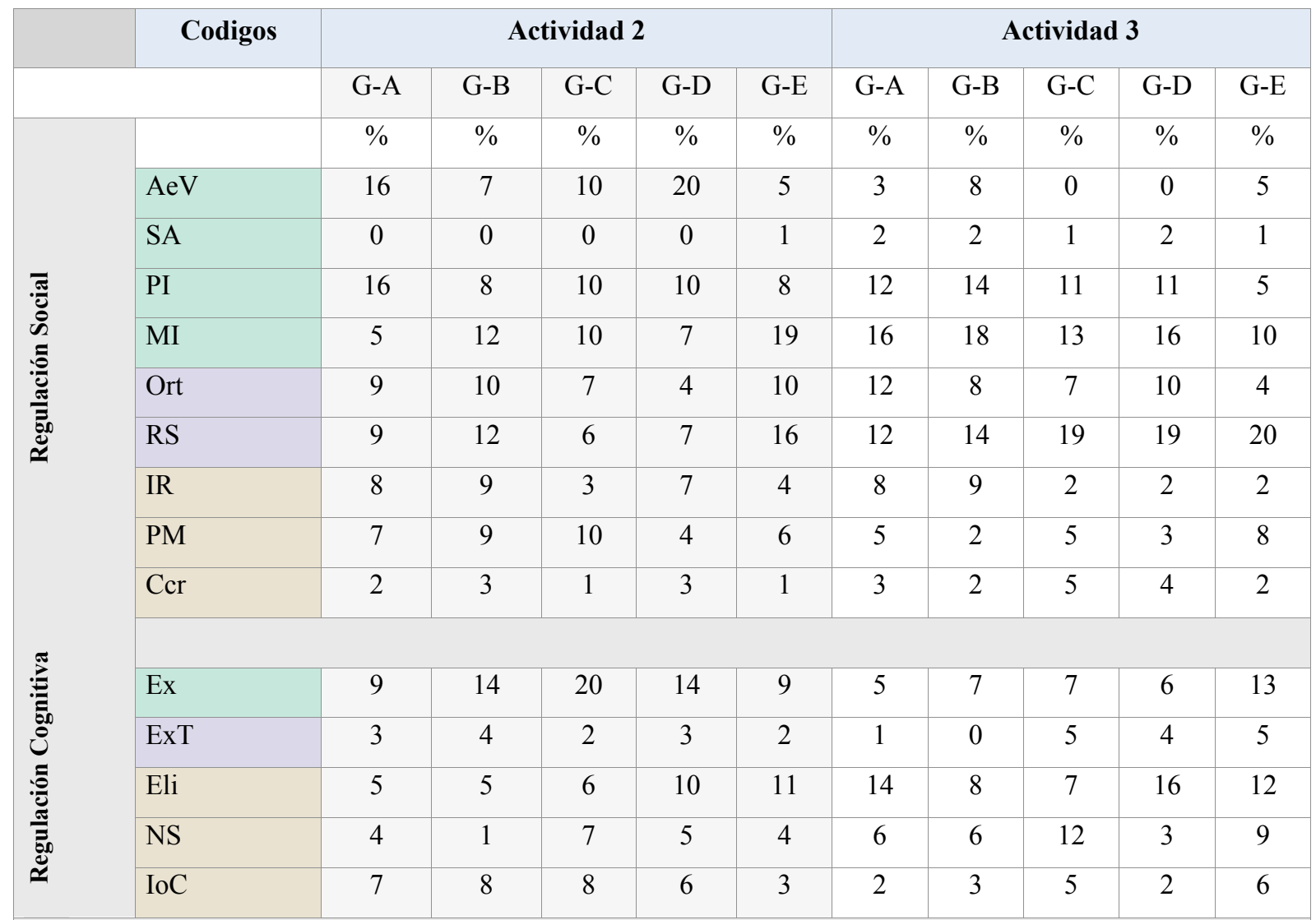

Nota: La tabla muestra los porcentajes correspondientes a la frecuencia con que se manifiesta cada una de las sub-categorías utilizadas para evaluar el esquema argumentativo de texto escrito en tarea cooperativa

G- Grupo

Autoreg. Reg. externa Reg.Conjunta

Tabla 15 Resumen de las frecuencias en porciento de las categorias de regulación utilizadas por los grupos en las actividades 2 y 3 . 
Las características en las interacciones en cada grupo puede presentar similitudes con la de otros, pero no significa que la regulación o la cooperación se haya desarrollado de la misma manera. A modo general, comparando los cinco grupos (Tabla 15), las categorías de la regulación social que más predominan a lo largo de ambas actividades son: Planificación individual (PI), Monitorización de su propia participación (MI), Estructurar la tarea (Ort), Refuerzos sociales (RS), seguidas de: Interpela responsabilidad/participación de los demás (IR) y Perspectiva mutua (PM). Las categorías con menos frecuencia de uso fueron: Situar el propio proceso de aprendizaje $(\mathrm{SA})$ y Concenso corto y rápido $(\mathrm{Ccr})$.

En cuanto a la frecuencia de las categorías de regulación cognitiva, se puede apreciar que Exteriorización (Ex) e Integración orientada al consenso (IoC) bajaron su frecuencia de uso en la actividad 3, mientras que la categoría Elicitación (Eli) y Negociar significados (NS) aumentó. La categoría Explicitar la tarea (ExT) no muestra cambios significativos.

Estos resultados concuerdan con las estrategias de regulación empleadas por los, analizadas en las encuestas realizadas previo al inicio de la asignatura. Los estudiantes enfatizaron en la importancia de tratar de cumplir con la planificación consensuada, en tratar de conectarse con frecuencia para ver las opiniones de los compañeros y dejar constancia de lo que cada cual esté realizando en el momento, en revisar exhaustivamente el requerimiento de la actividad a desarrollar. Además consideraron que se deben asumir roles como el liderazgo del grupo, proponiendo cronogramas de trabajo y enviando mensajes de motivación sin el ánimo de presionar a nadie. Otras estrategias se refieren a hacer diferentes propuestas para estimular la reacción de otros compañeros, asumir de manera consciente las propuestas sugeridas para incluir en el texto, apoyar a los compañeros de trabajo, dejar constancia sobre las cosas con las que se está de acuerdo y con las que no y proponer alternativas cuando se cree que favorecerán el desarrollo de la tarea. En las encuestas no hay evidencia de que se apliquen estrategias relacionadas con Situar el propio proceso de aprendizaje (SA) y de Concenso corto y rápido $(\mathrm{Ccr})$

Con respecto a las frecuencias de uso de la regulación cognitiva de una actividad a la otra, los grupos A, B y D, redujeron el uso de la categoría Exteriorización (Ex) a la mitad, el grupo C disminuye su uso a tres veces y el grupo E aumentó el uso de la categoría pero sin mayor significado. Mientras que la frecuencia de uso de la categoría Explicitar la tarea (ExT) en los grupos A y B tiende a desaparecer, en los demás grupos el uso de la categoría aumenta.

En cuanto a la frecuencia de uso de la categoría Elicitación (Eli), se puede apreciar que aumenta en el grupo D y aumenta al doble en el grupo A. En los demás grupos, la frecuencia 
de la categoría se mantiene constante. La categoría Negociar significados (NS) aumenta en todos los grupos, más significativamente en los grupos $\mathrm{C}$ y E. La categoría Integración orientada al consenso ( $\mathrm{IoC}$ ) disminuye su uso significativamente en los grupos A, B, D, en el grupo $\mathrm{C}$ disminuye la frecuencia de 8 a 5 , mientras que en el grupo $\mathrm{E}$ la frecuencia de uso se duplica.

Durante el análisis de las interacciones en ambas actividades, se observó un cambio general en la dinámica de la cooperación. Los mensajes de la fase de inicio en la actividad 3 se redujeron y tendieron a converger con los de la fase de intercambio. Ésta última también pareció acortarse, para dar paso directamente a la fase de negociación.

Se ha observado que la fase de negociación en la actividad 3 es más rica en contenido más crítica y mejor argumentada, sin embargo es menos numerosa en intervenciones que la actividad previa. La fase de aplicación aparece paralela a la fase de negociación y se alarga hasta la entrega del informe escrito. Es importante tener en cuenta que la posibilidad de que dos fases transcurran de modo paralelo es factible, puesto que la mayoría de los grupos organizó la dinámica de grupo por carpetas en el espacio de debate. De esta manera, se tenían carpetas exclusivas para el debate de contenidos y otra de cuestiones diversas, en las que se encontraban temas de organización grupal.

La cantidad de mensajes en cada fase y en cada actividad, se pueden apreciar en la siguiente figura.

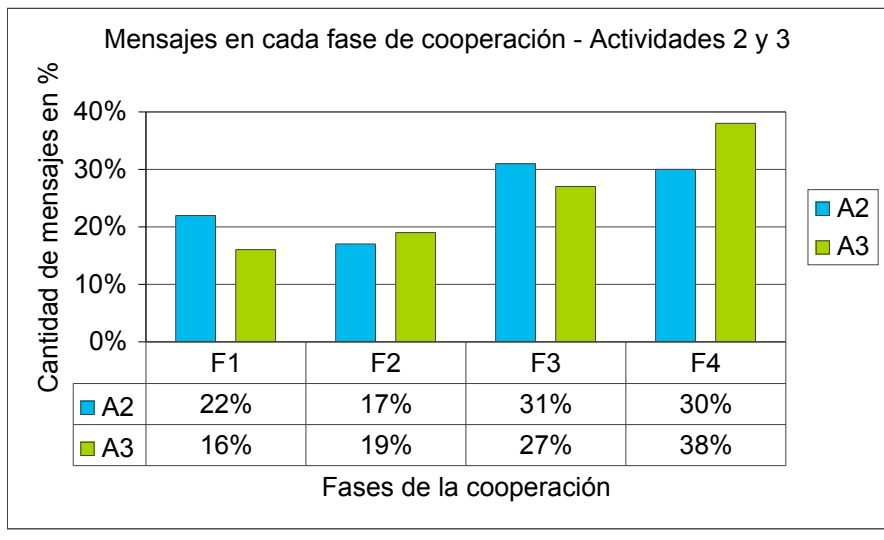

Fig. 6 Porcentaje de intervenciones en cada fase - Actividades 2 y 3

La observación acerca de la mejora en la calidad de los mensajes durante la tercera fase en la tercera actividad, concuerda con la impresión de la docente compartida antes de que ella realizara el análisis de los informes escritos, de que había una mejor regulación de las actuaciones de los estudiantes en la tercera actividad que también fue cooperativa y que mantuvo los mismos grupos de trabajo. La razón de esta mejora, puede relacionarse con el rol 
asumido por la docente durante el curso y al método evaluativo que aplica en las actividades, en las que ofrece un feedback bastante crítico del informe escrito, del proceso cooperativo de los estudiantes y además habilita un espacio de apoyo a la evaluación de textos escritos con la herramienta para la evaluación en línea annotation tool.

Según la información de la encuesta en línea, realizada al comienzo del curso acerca de las estrategias docentes que se consideraban útiles para ayudar a los estudiantes en tareas de escritura en línea, hay varios aspectos destacables. Se consideró como necasario ofrecer pautas orientativas, dar criterios claros, ofrecer orientación en el uso de herramientas colaborativas, ser breve, transparente, claro y concreto en las instrucciones e intervenciones.

En cuanto al seguimiento, los estudiantes dieron valor a cuestiones como apoyo, refuerzos sociales y sugerencias si se estimara necesario, comunicación amable pero a la vez exigente, que la retroalimentación se centre en las acciones y el proceso.

En el estudio realizado, entre las estrategias dialógicas docentes consideradas como importantes, se encuentran: la forma de expresión (saludo, tono, orden visual en el mensaje y despedida), expresar y hacer notar a los estudiantes que está presente y que sigue las acciones de los grupos.

Según los mensajes enviados al final de las actividades y luego de la retroalimentación ofrecida por la docente, se alcanza a percibir como los estudiantes valoran tanto el trabajo en grupo como el rol asumido por la docente. A continuación se presenta un mensaje del grupo E, expresando esta situación:

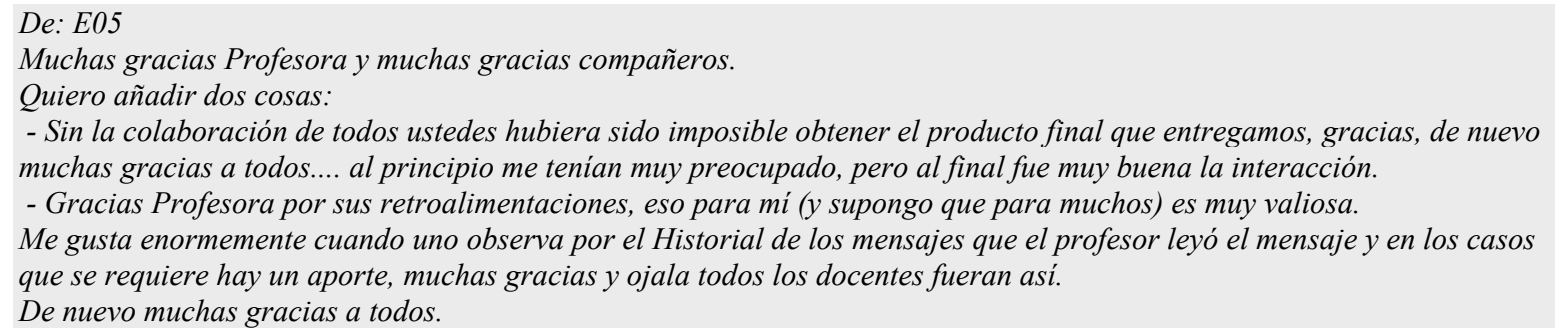

Si se asume que la calidad de los mensajes mejora de una actividad hacia la otra, también se asume que el resultado de aprendizaje reflejado en los informes escritos, ha mejorado. En el siguiente apartado se contrastan las observaciones e impresiones anteriores con el análisis de los informes escritos de cada uno de los grupos en ambas actividades. 


\subsection{Analisis 2: Contrastación del proceso de cooperación con los resultados del aprendizaje}

En este apartado se analizan los resultados de aprendizaje de cada grupo, se conoce la evolución de la calidad argumentativa de los informes escritos entregados en cada actividad. El análisis de los informes escritos de cada grupo, fue realizado por la docente, quien adaptó el aporte de Reznitskaya et al., (2008) sobre categorías para evaluar el esquema argumentativo del texto escrito en tareas cooperativas. Los informes textuales fueron análizados usando el programa científico para el análisis cualitativo de textos Nvivo QSR. Los criterios de evaluación empleados para éste objetivo y algunos ejemplos se adjuntan en el anexo F.

Con el resultado de los análisis, se contrastan las observaciones e impresiones de las interacciones durante las tareas de trabajo cooperativo.

El resumen del análisis de los textos de ambas actividades, ofrecido por la docente, se presenta en la Tabla 16.

La evolución de la calidad argumentativa de los informes escritos por los grupos en general es positiva, tal como se pensaba, sin embargo es necesario analizar a cada grupo por aparte. Para el criterio de Relevancia de ideas, conformado por las sub-categorías Ideas esenciales (IE) y Ideas no esenciales ( $\mathrm{InE}$ ), se observa que los grupos A, C y E se mantuvieron constantes a lo largo de los textos y que ninguno presentó una idea no esencial. El caso contrario es el del grupo B, que pasa de no tener idea no elaborada (InE) a tener un 22\%. El grupo D mostró un $14 \%$ de ideas no elaboradas en su primer informe y en el segundo este porcentaje se duplicó, indicando el empeoramiento en la relevancia de ideas expuestas.

En cuanto a la focalización de las ideas esenciales, representado por Argumentos Relevantes (AR) y Argumentos circunstanciales (AC), se observa una evolución positiva en los grupos A, C y E. El segundo informe de estos grupos no manifiesta la presencia de la categoría AC relacionada con enunciados superficiales que son poco o nada ilustrativos, mientras que el grupo D además de presentar en su primer informe un 35\% de argumentos circunstanciales (AC), en el segundo informe éstos se aumentan a un 56\%. El grupo B, no muestra cambios en la manifestación de ésta categoría, en ambos informes el porcentaje de argumentos circunstanciales (AC) se mantiene alrededor de un $20 \%$. 


\begin{tabular}{|c|c|c|c|c|c|c|c|c|c|c|c|c|c|c|c|c|c|c|c|c|c|c|c|c|}
\hline & \multirow{2}{*}{\multicolumn{2}{|c|}{ Densidad del texto }} & \multicolumn{4}{|c|}{ Relevancia } & \multicolumn{4}{|c|}{ Focalización } & \multicolumn{8}{|c|}{ Soporte de las ideas } & \multicolumn{6}{|c|}{ Elaboración } \\
\hline & & & \multicolumn{2}{|c|}{$\begin{array}{c}\text { Ideas } \\
\text { Esenciales }\end{array}$} & \multicolumn{2}{|c|}{$\begin{array}{l}\text { Ideas no } \\
\text { Esenciales }\end{array}$} & \multicolumn{2}{|c|}{$\begin{array}{l}\text { Argumentos } \\
\text { Relevantes }\end{array}$} & \multicolumn{2}{|c|}{$\begin{array}{l}\text { Argumentos } \\
\text { Irrelevantes }\end{array}$} & \multicolumn{2}{|c|}{ Textual } & \multicolumn{2}{|c|}{ Hipotética } & \multicolumn{2}{|c|}{ Abstracta } & \multicolumn{2}{|c|}{ Contextual } & \multicolumn{2}{|c|}{ Elaborada } & \multicolumn{2}{|c|}{$\begin{array}{c}\text { Poco } \\
\text { Elaborada }\end{array}$} & \multicolumn{2}{|c|}{ No Elaborada } \\
\hline & A2 & $\mathbf{A 3}$ & $\mathbf{A 2}$ & A3 & A2 & A3 & $\mathbf{A 2}$ & A3 & A2 & A3 & A2 & A3 & $\mathbf{A 2}$ & A3 & A2 & A3 & A2 & $\mathbf{A 3}$ & A2 & A3 & $\mathbf{A 2}$ & A3 & A2 & A3 \\
\hline G-A & 6,533 & 6,877 & 100 & 100 & 0 & 0 & 73 & 100 & 27 & 0 & 23 & 0 & 21 & 10 & 27 & 49 & 29 & 41 & 48 & 100 & 43 & 0 & 9 & 0 \\
\hline G-B & 5,836 & 9,214 & 100 & 78 & 0 & 22 & 79 & 78 & 21 & 22 & 54 & 40 & 7 & 2 & 21 & 37 & 18 & 22 & 10 & 14 & 67 & 64 & 24 & 21 \\
\hline G-C & 2,901 & 1,650 & 100 & 100 & 0 & 0 & 93 & 100 & 0 & 0 & 20 & 6 & 12 & 11 & 37 & 22 & 32 & 61 & 90 & 75 & 10 & 25 & 0 & 0 \\
\hline G-D & 4,856 & 14,4004 & 86 & 68 & 14 & 32 & 65 & 44 & 35 & 56 & 37 & 49 & 15 & 4 & 30 & 26 & 17 & 20 & 67 & 23 & 17 & 54 & 17 & 23 \\
\hline G-E & 4,386 & 5,800 & 100 & 100 & 0 & 0 & 100 & 100 & 0 & 0 & 26 & 6 & 16 & 6 & 32 & 26 & 26 & 62 & 100 & 100 & 0 & 0 & 0 & 0 \\
\hline
\end{tabular}

Nota: La tabla muestra los porcentajes correspondientes a la frecuencia con que se manifiesta cada una de las sub-categorías utilizadas para evaluar el esquema argumentativo de texto escrito en tarea cooperativa. A2: Actividad 2, A3: Actividad 3, G- : Grupo

Tabla 16 Resumen del análisis de los textos elaborados durantes las actividades

a)

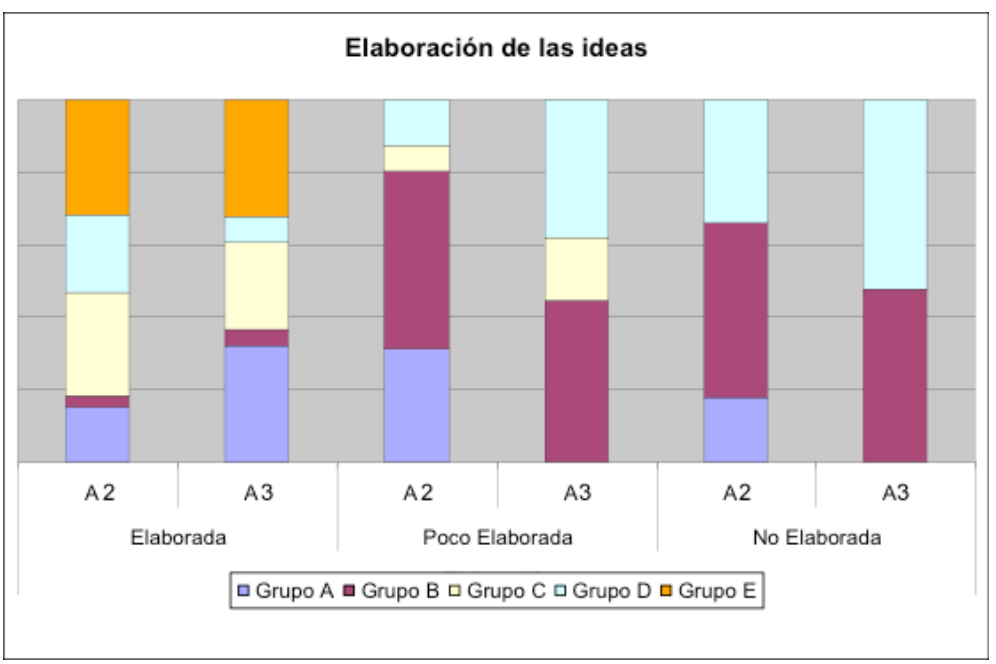

b)

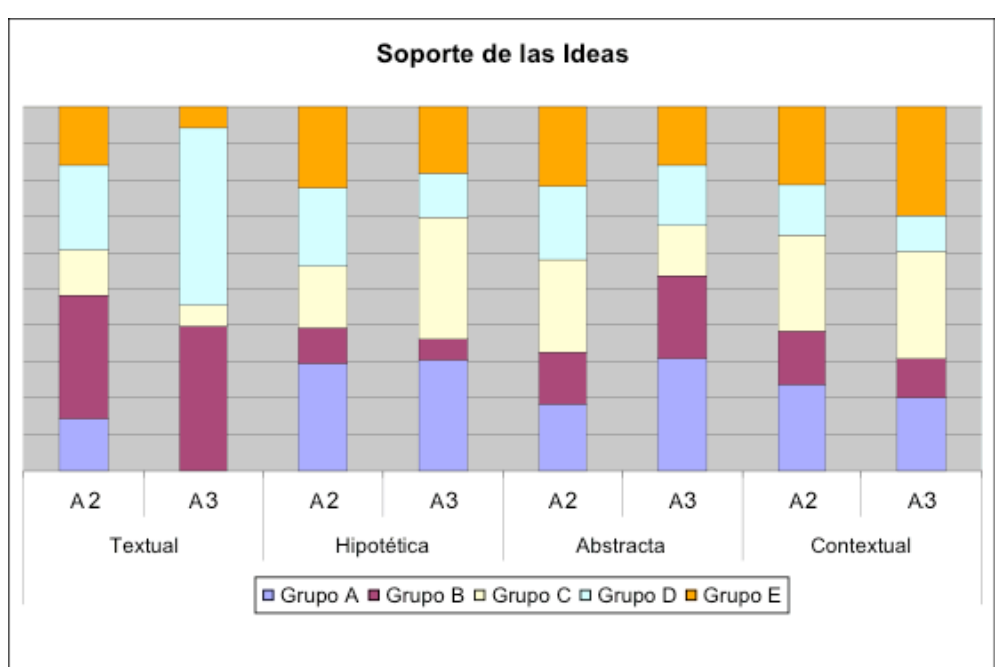

Nota: A2: Actividad 2, A3: Actividad 3

Fig. 7 Resumen contrastado por grupos de las dos actividades en estudio por criterios de: a) Elaboración de ideas y b) Soporte de ideas 
La categoría Soporte de las ideas, comprende las sub-categorías Textual (TxT), Hipótesis (Hp), Abstracción (Abs), Contextualización (Ctxt) y se manifiesta en los grupos de la siguiente manera: El grupo A muestra una evolución positiva al duplicar los valores de las sub-categorias Abs y Ctxt, referidas respectivamente a la generalización, prescripción sobre cómo actuar o sobre las consecuencias de la actuación y a la reconstrucción de la situación, atendiendo al contexto, la audiencia, etc.

El grupo B evoluciona mostrando menos soportes textuales (TxT) y más abstracciones (Abs), en el grupo $\mathrm{C}$ se observa como la sub-categoría de contextualización (Ctxt) se duplica favorablemente, disminuyendo el uso de soportes de carácter textual (Txt). El grupo D aumenta el uso soportes textuales (Txt) de un 37\% a un 49\% y reduciendo el uso de la subcategoría de hipótesis de un $15 \%$ a un $4 \%$. El grupo E aumenta considerablemente a un $62 \%$ el uso de la sub-categoría de contextualización (Ctxt) que en el primer informe apenas alcanzaba un 26\%, con esto bajó el porcentaje de las sub-categorías de soporte textual (TxT) e hipotético (Hp) a un 6\% cada una.

La última categoría evaluada se refiere a la elaboración y comprende las sub-categorías Ideas elaboradas (Ielab), Ideas incompletas (Pelab) e Ideas no elaboradas (Nelab). En esta categoría se evidencia que el Grupo E se mantuvo constante a lo largo de ambos informes con un 100\% en Ideas elaboradas (IElab). El grupo A muestra una evolución positiva y alcanza todo el porcentaje de Ideas elaboradas (IElab) en su segundo informe, en cambio el grupo C disminuye el porcentaje en esa categoría pero aumenta el porcentaje de ideas incompletas de un $10 \%$ a un $25 \%$. En ambos informes del grupo $\mathrm{C}$ no se manifiestan Ideas no elaboradas (NElab). El grupo D pasa de tener un 54\% de ideas elaboradas (IElab) en su primer informe a un $23 \%$ en su segundo informe, aumentado así el porcentaje de Ideas incompletas (PElab) de un $17 \%$ a un $54 \%$, manteniendo un porcentaje de Ideas no elaboradas (NElab) constante alrededor del 20\% durante ambos informes. El grupo B mantiene los porcentajes constantes durante ambos informes, sin manifestar un cambio significativo.

Con los resultados de los informes, se puede ver como el trabajo de los grupos A, C y E muestran una evolución positiva en cuanto al esquema argumentativo empleado, mientras que en el grupo D se evidencia una retro-evolución. El grupo B no muestra cambios significativos, más bien mantiene los porcentajes constantes en ambos informes.

Si se observa la cantidad de palabras empleadas en los informes de cada grupo, se puede apreciar en general que los segundos informes aumentaron su cantidad, excepto en el grupo C en el que se disminuyeron a casi la mitad. La extensión de los informes, como se pudo 
apreciar en los resultados de la evaluación del esquema argumentativo, no comprueban la riqueza en contenido. El grupo D por ejemplo utilizó 3 veces más la cantidad de palabras utilizadas en el primer informe y según las conclusiones de los resultados anteriormente descritos, este grupo envez de mostrar una evolución positiva, demostró todo lo contrario. El grupo B que también incrementó el número de palabras en un 57\%, no muestra una evolución durante ambos informes, sino que sus valores se mantuvieron siempre constantes y su esquema argumentativo no siguió un buen modelo. La extensión de los informes de los grupos A y D guardan una relación y según el análisis del esquema argumentativo, éstos grupos mostraron una evolución bastante positiva. Aunque la extensión del segundo informe del grupo C disminuyó un $43 \%$ con respecto al primer informe entregado, los resultados de la calidad argumentativa de los informes muestran que éste grupo también se desarrolló positivamente.

Con esta observación no solo se pueden comprobar las observaciones del análisis anterior, sino que se puede concluir además cuáles fueron grupos en los que se pudo haber detectado una mejora en su cooperación y cómo esto fue plasmado en el informe final.

\subsection{Análisis 3: Modelos de regulación durante tareas cooperativas en entornos virtuales asincrónicos y escritos}

Mediante los análisis previos, fue posible explorar modelos de interacción representativos en cada fase de trabajo cooperativo, en los que identifican indicadores para observar y evaluar la regulación del aprendizaje. En este análisis se consideró todo el proceso de los grupos durante ambas actividades. Se analizaron los mensajes que constituían cada interacción, y en cada mensaje se evaluaron sus unidades temáticas.

Algunas de las unidades temáticas combinaron ambas categorías de la regulación social y cognitiva. En total se encontró el uso de 40 categorías en las unidades temáticas estudiadas durante todas las fases cooperativas. Para decidir cuáles categorías representaban a cada fase, se tuvieron en cuenta dos criterios: que la categoría estuviera presente en ambas actividades y que la frecuencia de unidades temáticas con esa categoría fuera mayor de 15 veces en alguna de las dos actividades. La frecuencia de unidades temáticas con la que cada categoría aparece en la Figura Fig. 8 resulta de la suma de las frecuencias en ambas actividades.

El resultado de las categorías para las fases de la cooperación se presenta en la Figura Fig. 8. 


\begin{tabular}{|c|c|c|c|c|c|c|c|c|c|c|c|c|}
\hline \multirow[b]{2}{*}{ Fase } & \multirow[b]{2}{*}{ Act } & \multicolumn{4}{|c|}{ Social } & \multicolumn{3}{|c|}{ Combinada } & \multicolumn{4}{|c|}{ Cognitiva } \\
\hline & & OrT & MI & $\mathrm{RS}$ & PI & IR & $\begin{array}{c}\mathrm{AEV}- \\
\mathrm{EX}\end{array}$ & $\begin{array}{c}\text { PM - } \\
\text { EX }\end{array}$ & $\mathrm{IoC}$ & NS & ELi & EX \\
\hline \multirow{2}{*}{1} & $\mathrm{~A}$ & 27 & & 22 & 26 & & & & & & 16 & 19 \\
\hline & $\mathrm{B}$ & 6 & & 13 & 11 & & & & & & 6 & 1 \\
\hline \multirow[t]{2}{*}{2} & $\mathrm{~A}$ & & 18 & & & & 15 & & & & 19 & 44 \\
\hline & B & & 11 & & & & 2 & & & & 13 & 10 \\
\hline \multirow{2}{*}{3} & $\mathrm{~A}$ & & 32 & & & & & 16 & 61 & 35 & 27 & 87 \\
\hline & B & & 17 & & & & & 4 & 17 & 17 & 21 & 18 \\
\hline \multirow{2}{*}{4} & $\bar{A}$ & 22 & 477 & $\overline{52}$ & 47 & 28 & & & & & 21 & 2 \\
\hline & B & 13 & 38 & 62 & 23 & 15 & & & & & 28 & 18 \\
\hline
\end{tabular}

$\mathrm{n}=1434 \mathrm{TU}$ :

Nota: Las barras de los gráficos demuestran los lenguajes de la regulación en cada fase cooperativa, los códigos se describen en el Anexo H. La fuente de los datos es la totalidad de unidades temáticas de todos los grupos en las actividades 2 y 3 . Act: Actividad.

Fig. 8 . Comportamiento de la regulación del aprendizaje en las fases de cooperación

A continuación se describen las características encontradas en cada fase cooperativa.

Fase 1: Inicio (ver Fig. 8)

En esta primera fase de trabajo cooperativo, las categorías de regulación emergentes de los datos son Estructurar la tarea (Ort) con 33 UT, Refuerzos sociales (RS) con 35, PI (Planificación individual) con 37, Exteriorización (EX) con 20 y Elicitación (Eli) con 22.

Si se presta atención a estos resultados, se puede ver que la frecuencia de las últimas dos categorías EX y Eli, son las más bajas. Esto se puede explicar por las características de ésta fase (Casanova, 2008), en la se supone el grupo apenas está organizándose, definiendo la tarea, en donde surgen propuestas para enfocar su realización. Durante la investigación, se observó que las intervenciones en esta fase eran principalmente de carácter individual, ya sea para mostrar la asunción de compromisos y para estimular su integración con los demás miembros del grupo mediante lenguajes que generaran diálogo social.

Algunos ejemplos encontrados en esta primera fase son de los grupos A y B:

De: $E 03$

Hola a todos,
Pues aquí estoy dispuesto a trabajar con vosotros en este caso.

¿Empezamos a organizarnos con "las reglas del juego"?

Cordiales saludos,

De: E04

A

A trabajar jejejejej!!!!

$\mathrm{De}: \mathrm{EO}$

Perfecta entrada en materia

¿que os parece si a la planificación de E02 le añadimos una estructura de plazos y responsables y nos dedicamos a profundizar en el tema de la organzación para que

también constituya un elemento de nuestro proceso de elaboración del informe?

Podíamos ir viendo en que rol nos sentimos más comódos y nos vamos ofreciendo... si no hay coincidencias pues... adelante y si las hay ... pues vemos como solucionar la

De esta forma, podemos compartir las funciones y los plazos e ir retroalimentando según vaya desarrollandose el debate... 
Fase 2: Intercambio (ver Fig. 8)

En la segunda fase de trabajo cooperativo se evidencian tres categorías individuales y una categoría combinada. La fase de intercambio se caracteriza por intervenciones que reflejan una mejor familiarización con el grupo y con la tarea a realizar. El lenguaje predominante es acumulativo y aunque los aportes en esta fase favorecen positivamente para la realización de la actividad, no son aportes ni críticos (Casanova, 2008), ni argumentados.

La combinación de las categorías Auto-evaluación (AEV) y Exteriorización (EX) en una misma unidad temática se considera destacable e importante, puesto que así es posible conocer cómo los estudiantes relacionan lo que ya saben, conocen o han experimentado con la tarea a realizar y también para conocer, por parte de los estudiantes, si la realización de la tarea le traerá beneficios para su contexto cotidiano. Como se puede observar, esta categoría, aunque baja en frecuencia (17 UT), logra sobresalir como categoría modelo, seguida de Monitorización de su propia participación (MI) con 29 UT, Elicitación (Eli) con 32 UT y Exteriorización (EX) con 54 UT.

La categoría con mayor frecuencia es la de Exteriorización (EX), que demuestra como los estudiantes se van familiarizando con los contenidos. Los estudiantes aportan información textual o expresan sus puntos de vista sin necesariamente hacer referencia a algún aporte anterior. Aquí se busca conocer las opiniones de otros compañeros, mediante la Elicitación (Eli). La regulación en esta fase oscila entre los modos auto- y externo.

Algunos ejemplos encontrados en esta segunda fase son de los grupos: A, B, E, C

$$
\text { De: E06 }
$$

Hola compañeros,

Qué les parece si empezamos a aportar sobre los primeros puntos que nos piden sobre el caso elegido

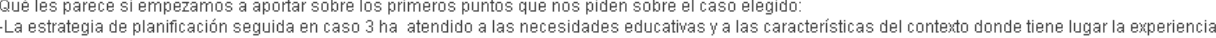

Pienso que si, se trabaja con un modelo pedagógico -tecnologico que se centra en el estudiante. Prima la flexibilidad en el proceso de enseánza - aprendizaje, el docente actua como

guia del alumno permitiendo un aprendizaje autodidactico pero con asesoramiento continuo.

ara entrar en contexto, quisiera que alguno me ayudara en lo siguiente; cuando se dice que el caso 3 es una entidad dedicada a la formación universitaria de primer y tercer ciclo en el ámbito de la acción social, que significa

Primer y tercer ciclo, existe algún segundo ciclo

Cuando dice que ámbito de la acción social, se refiere a los programas que ofrece o la población?

E04

De: E01

Hola buen día a todo

Dados mis compromisos laborales, también me inclino por el caso 3 , sin embargo, adhiero a la decisión que el grupo asuma

EXX, el caso 1 es interesante, tal vez en nuestr país lenemos experiencias en ese sentido, basta con revisar un poco lo que ha trabajado la universidad Xxox alrededor del proyecto Cordialmente

Cor

Cordial saludo para todos.

Mis disculpas por la ausencia,

concentrada en el proceso de trabajo de construcción grupa

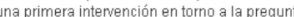

accesos de enseñanzay aprendizaje con la introducción de las tecnologías (TIC) para el caso concreto caso 37

Esta planif ipotante es que el proceso educativo virtual exige mayor planificación, mayor acuerco entre los distintos participantes para que se lleve a cabo de manera adecuada

momentos de la formación. 


\section{Fase 3: Negociación (ver Fig. 8)}

La tercera fase de trabajo cooperativo, es la fase de negociación (Casanova, 2008) que se caracteriza por la construcción mutua de significados y por un lenguaje de mayor valor cognitivo. En esta fase, aunque también con baja frecuencia, se encontró la combinación de dos categorías de la regulación: Perspectiva mutua (PM) con Exteriorización (EX). Esta combinación resulta luego de que el estudiante ha interpretado el mensaje de otro compañero, de querer mostrar su acuerdo y de reforzar esa idea desde su propio punto de vista o con ideas textuales encontradas en el contenido.

La categoría Monitorización de su propia participación (MI) se interpreta como la forma que un estudiante tiene de expresarle al grupo que se encuentra activo e implicado en la tarea, mediante mensajes que describen esa implicación o adjuntos que demuestran su trabajo.

Así como en los resultados del estudio de Casanova, en este análisis las categorías de regulación más implicadas durante esta fase son las cognitivas: Elicitar (Eli) con 48 UT, Negociar significado (NS) con 52 UT, Integración orientada al consenso (IoC) con 78 UT y con mayor frecuencia Exteriorización (EX) con 105 UT.

Esta fase se caracteriza por la dinámica de grupo, el diálogo grupal, la construcción conjunta de significados. Las interacciones de éste tipo suelen comenzar con una propuesta argumentada, a modo de negociación, algunas opiniones personales para reforzar lo escrito, concluyendo el mensaje con una Elicitación (Eli), para animar a continuar la discusión. El desencadenamiento de las interacciones posteriores, pueden contener mensajes con unidades temáticas como Perspectiva mutua (PM) con Exteriorización (EX), también pueden proponer algo diferente mediante una nueva negociación Negociar significado (NS); además puede manifestar acuerdo mediante la integración de ideas o intervenciones de los demás (IoC) ya sea con un mensaje o con un documento adjunto. Como el grupo es el que construye, se evidencia la necesidad de reacción u opinión de los demás respecto a lo construido o acordado, y esto se realiza mediante una Elicitación (Eli).

Un ejemplo de interacción grupal (grupo E) y de construcción de conocimiento en esta tercera fase es la siguiente:

Nota: La versión para esta fase se ha acortado, por su extensión. Sin embargo es posible reconocer los tipos de lenguajes utilizados en las interacciones con su respectiva codificación original. 


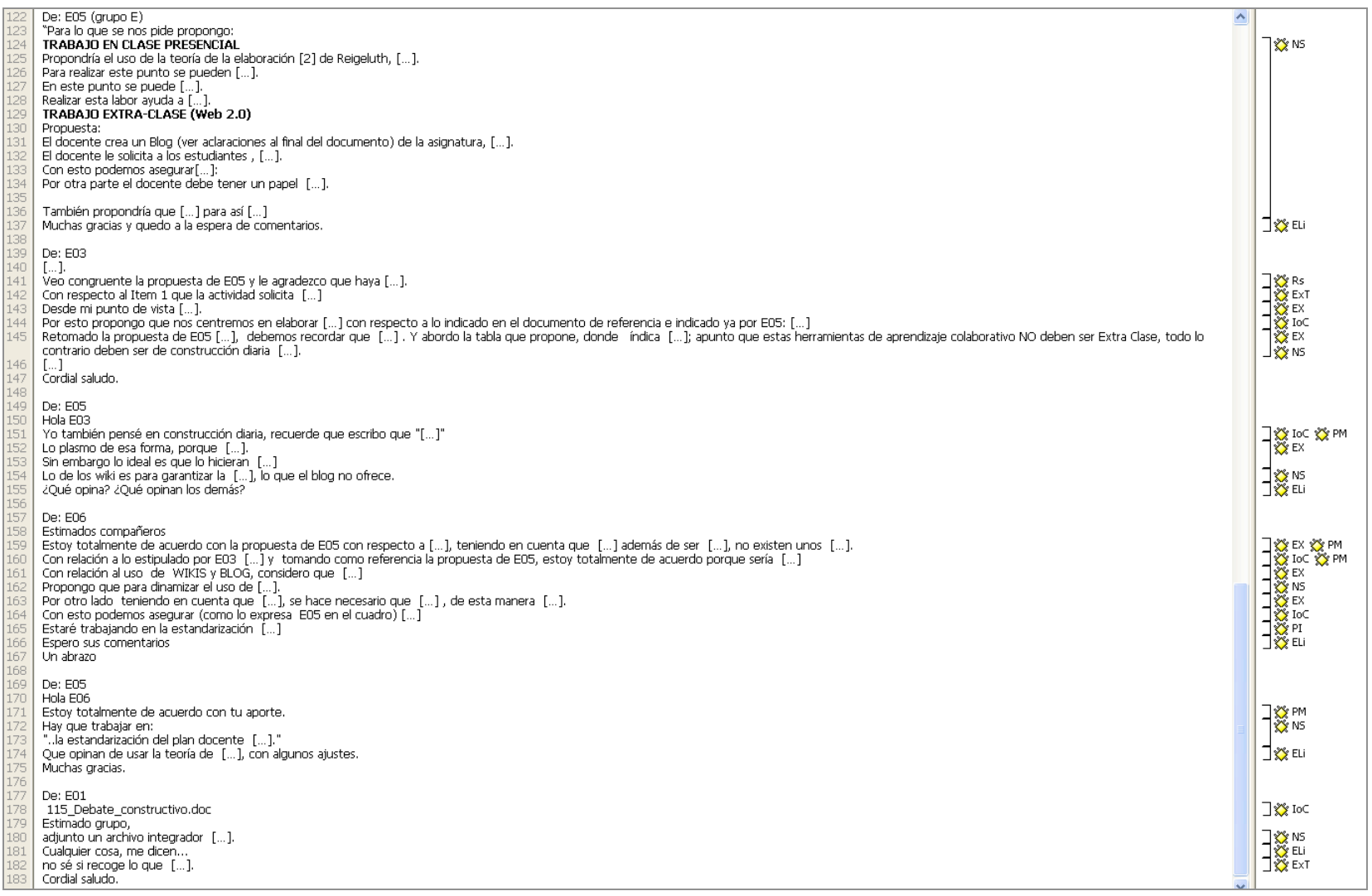

Fase 4: Aplicación (ver Fig. 8)

En la cuarta y última fase cooperativa (Casanova, 2008) se concreta y se aplica lo que se ha intercambiado en las fases previas mediante la realización del producto final requerido, en nuestro caso un informe escrito.

Las categorías que más sobresalen en esta fase son las de regulación social, representada con la categoría Refuerzos sociales (RS) con 114 UT. Esto puede explicarse por la larga trayectoria de 4 semanas de trabajo que cada grupo ha invertido en cada actividad. Mientras que la mayoría de integrantes del grupo se preocupan por la presentación del producto final y de la integración coherente de ideas, hay otros estudiantes que continúan con la Exteriorización (EX), esta vez de aseveraciones cognitivas que ilustran la modificación en sus esquemas cognitivos (Gunawardena et al., 1997) como resultado del proceso de interacción. La constancia y responsabilidad de todos los miembros para con el grupo se representa mediante las categorías Monitorización de su propia participación (MI) y Planificación individual (PI).

Un ejemplo de interacción grupal (grupo C) en la cuarta fase de trabajo cooperativo es: 
De: E04

Actividad3-Subqrupo1.doc

Hola equipo,

Con las aportaciones que se habian hecho hasta el momento, más las mías propias, he redactado una primera versión de lo que podría

ser nuestro documento final.

Está en Google Docs, claro, no obstante lo adjunto aquí también para que nos hagamos a la idea de como está quedando.

Un cordial saludo

De: $\mathrm{E} 01$

Hola E02, estaba leyendo la versión de la actividad que has colgado... te he ido a hablar por el messenger pero ya habias desaparecido.

todo el trabajo me parece bien,

pero quería desarrollar el último punto "Establecimiento de objetivos específicos que siman para interpretar los resultados" Pero es que

no entiendo muy bien qué hay que desarrollar en ese puntos: ¿una serie de estándares para la evaluación, es decir, unos valores de

referencia en relación a cada uno de los puntos en los que hemos centrado la evaluación?

De: E03

Ese punto lo había incluido yo, pero simplemente como una idea que había que considerar,

no creo que sea necesario mantenerlo como un punto.

De: E02

Hola E04

$\mathrm{Si}$, el documento es una recopilación de todas las ideas que hemos escrito en Google Docs, y está en word para que nos hagamos una

idea de como va quedando.

Pero creo que lo suyo es seguir con las aportaciones en Google Docs entre lo que queda de hoyy mañana de cara a enviarlo mañana.

Cordial saludo,

De: E01

Hola E02 y amigos del grupo,

Por mi parte expreso que se han estructurado todas las ideas planteadas

sólo me queda agradecer a todos.

De: E03

He estado revisando y haciendo algunos cambios en el documento.

E02, ite encargas tú de enviarlo?

De: E02

Por supuesto, ahora mismo me encargo.

Cordial saludo,

De: E02

Actividad 3

Hola,

Pues si todos estais de acuerdo, esta si que sería la versión definitiva del documento.

Respecto a la versión de ayer, he introducido los comentarios de E03y alguno mío, además de retocar un poco el documento en general.

Por favor, decidme algo, y si no,

en media hora a lo sumo, se la envío a la profesora.

Cordial saludo y gracias por el trabajo realizado,

De: E02

Hola a todos de nuevo,

Acabo de enviar la actividad en el espacio de "Entrega"

Cordial saludo,

De: E03

¡Gracias E02

Ha sido un verdadero placer trabajar contigo.

Con la descripción del comportamiento de la regulación en las fases cooperativas en debates en entornos virtuales, así como con la presentación de algunos ejemplos representativos en cada fase y tomando como base el marco teórico de trabajo de Volet, Summers yThurman (2008) y las fases de trabajo cooperativo de Gunawardena et al., (1997) adaptadas por Casanova (2008), se concluye este apartado con la integración del resultado del análisis mediante la elaboración de un modelo (Fig. 9).

En el modelo se propone representar la evolución de las fases de la cooperación sobre las dimensiones de la regulación social y cognitiva. Por medio de las interacciones durante las fases se puede confirmar que la regulación social ocurre en los tres modos: auto-, externa y conjunta como lo afirmó Vauras et al (2003) y las cuatro fases cooperativas adaptadas por 
Casanova (2008), se ven representadas en los cuatro cuadrantes propuestos por Volet et al (2008).

Esta propuesta intenta mostrar que las fases son progresivas, y que cada fase depende de la fase anterior. Así, la cooperación de un grupo comienza en el tercer cuadrante, correspondiente a la fase 1 de Inicio, continúa con la segunda fase de Intercambio en el segundo cuadrante, seguida de la fase 3 de Negociación en el primer cuadrante, y concluye en el cuarto cuadrante, con la fase 4 de aplicación.

La fase cooperativa de mayor interés para la evaluación de la regulación de la construcción del aprendizaje, es la tercera fase como lo propone Casanova (2008). En ésta fase además de evidenciarse la presencia de la corregulación, emergen lenguajes más ligados a la construcción de significado. Esto no significa representa que las demás fases no sean importantes, al contrario, todas éstas constituyen la base del trabajo cooperativo y las cuatro fases comprenden un solo proceso.

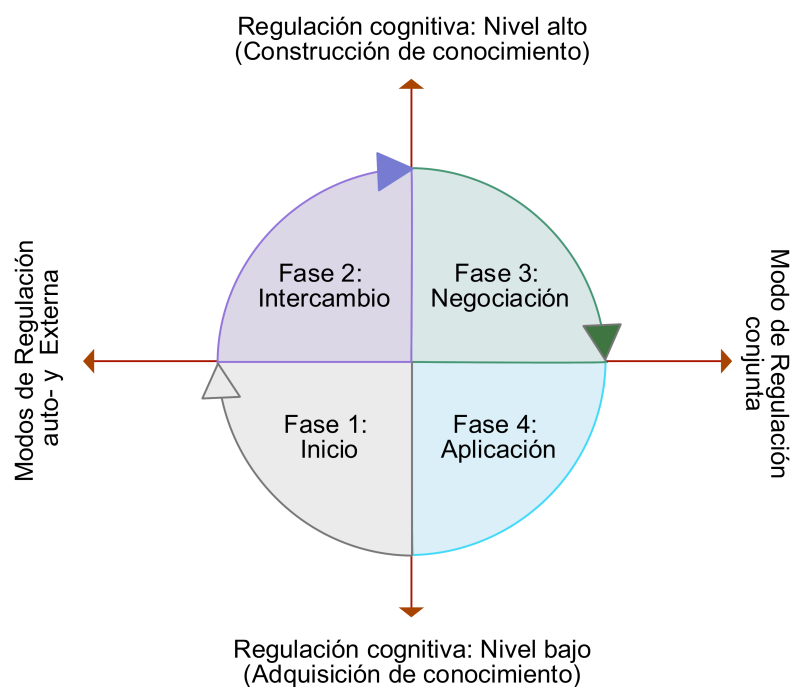

Fig. 9 Modelos de regulación: Comportamiento de la regulación durante tareas cooperativas virtuales 


\section{Conclusiones}

Una vez presentado y discutido, tanto el marco teórico de referencia como los resultados de la investigación llevada a cabo, se destacan a continuación las principales conclusiones que se desprenden del trabajo.

Para facilitar la comunicación se toman como referencias el objetivo propuesto y las diversas preguntas que guiaron el estudio. De este modo las conclusiones que se presentan constituyen una aproximación conceptual a la pregunta central de la investigación que se responde al final de este apartado a modo de propuesta educativa.

En primer lugar, se hace necesario identificar cuáles son las estrategias que utilizan los estudiantes para regular sus intervenciones durante la cooperación.

En el estudio realizado, mediante el análisis del lenguaje, más exactamente del discurso utilizado por los estudiantes en los mensajes que intercambian, se hizo posible identificar las estrategias de regulación utilizadas durante el desarrollo de tareas cooperativas escritas (Mercer, 2004; Vygotsky, 1979 \& Weinberger et al., 2006).

Los resultados reflejan que los estudiantes implementan tanto estrategias reguladoras de nivel social como de nivel cognitivo, coincidiendo así con los hallazgos de Volet et al., (2009) en su estudio de estas cuestiones en tareas cooperativas entornos universitarios presenciales.

En relación con la regulación social y cognitiva las observaciones realizadas al proceso de cooperación durante la realización de tareas de escritura ponen de manifiesto estrategias funcionales, contribuyentes a la regulación de la cooperación y de la construcción conjunta del conocimiento. Para la regulación social se destacan estrategias tales como: situar el propio proceso de aprendizaje, realizar la auto-evaluación, monitorizar la participación, planificación individual (Autoregulación); estructurar la tarea, ofrecer refuerzos sociales (Regulación externa); contribuir al consenso corto y rápido, la perspectiva mutua, interpelar responsabilidad y/o participación de los demás (Regulación conjunta).

Para la regulación cognitiva se destacan estrategias como: Exteriorizar conocimientos y puntos de vistas previos (Autoregulación), explicitar la tarea (Regulación externa), elicitar opiniones o cuestionamientos, negociar significados y realizar esfuerzos de integración de aportaciones orientado al consenso (Regulación conjunta).

Nos parece importante acotar que esta investigación retoma y esclarece los indicadores del lenguaje de la cooperación que aportó el estudio de Casanova (2008) y aporta/complementa 
los lenguajes que dan cuenta de la regulación cognitiva (dimensión de construcción conjunta de la cooperación), que históricamente han sido menos abordados en investigaciones al respecto.

En segundo lugar y teniendo en cuenta este primer resultado se procedió a la exploración de modelos de interacción durante las tareas cooperativas estudiadas. Así se pudo identificar un modelo que muestra el comportamiento de estas estrategias de regulación a lo largo de la realización de una tarea cooperativa, con demanda argumentativa, en un entorno virtual asincrónico y escrito. Este resultado corrobora la segunda hipótesis que se plantea para esta investigación.

El análisis realizado permite constatar que los lenguajes varían en función a la fase de cooperación en la que el grupo se encuentre, en unas más intensa que otras. Es interesante ver que en este contexto y durante todas las fases de cooperación predomina el lenguaje que manifiesta la elicitación, lo que puede se puede interpretar como un grado alto de implicación de los estudiantes en la tarea cooperativa a través del que buscan el diálogo y la construcción con sus compañeros. Además, la elicitación se interpreta como la forma en que los estudiantes valoran a sus compañeros por las experiencias y conocimientos previos que puedan aportar tanto para la resolución de la tarea como para la experiencia propia.

Otra estrategia que demuestra la implicación de los estudiantes durante las distintas fases de trabajo, son las de carácter individual (monitorización individual y planificación individual), con las que los estudiantes hacen notar su presencia en el grupo, la asunción de responsabilidades, la importancia que le dan al trabajo en grupo y justifican cada acción y movimiento realizado. (Pintrich, 2000; Schunk \& Zimmerman (1994); Vygotsky (1979); Whipp \& Chiarelli (2004); Zimmerman , 1995, 1997)

Los lenguajes que muestran refuerzos sociales, aunque de valiosa importancia en contextos virtuales, como plantea Casanova (2008) aparece en nuestro análisis más ligada en las fases cooperativas de inicio y aplicación donde la concentración del grupo no se enfocaba hacia el contenido sino más bien a la organización del grupo y a la presentación del producto final creado conjuntamente.

El sentimiento de "pertenencia" al grupo y de autovalorar que sus aportes son importantes para la construcción conjunta, en beneficio de todos, se ve reflejado mediante la libertad de expresión por medio de la exteriorización. 
La negociación aparece como una necesidad de diálogo, pero enfocado hacia el contenido. A partir de los conocimientos previos y adquiridos, y experiencias propias, cada estudiante se arma de argumentos no sólo para presentar una propuesta sino también para defenderla. En la negociación los estudiantes son conscientes de que el producto a crear solo es provechoso si todos han opinado, además se sabe que las ideas se pueden mejorar, construir, recontruir, reelaborar, desarollar, mediante esta estrategia. De esta manera es como el razonamiento se va conformando por el flujo de proposiciones dentro de discursos con razones argumentadas (Reznitskaya et. al, 2008).

La combinación de estrategias para autoevaluar el aprendizaje y para exteriorizar el conocimiento o la perspectiva propia, aunque baja en frecuencia de uso, refleja una estrategia usada por los estudiantes en la fase crucial de intercambio, en las que se hace necesario que cada cual valore lo que sabe o puede y cómo lo puede integrar o relacionar para la resolución de la actividad. El uso/ buen empleo de esta estrategia, al igual que las estrategias para situar las metas de aprendizaje, que no apareció como dominante en los resultados, podría favorecer todavía más al mejor desarrollo de la fase de negociación, que necesita concentración y enfoque hacia los objetivos específicos de la tarea a resolver.

Las estrategias para la integración de contenidos que se intercambian y discuten se puede considerar como las más importantes, puesto que indica que también se utilizan otras como exteriorizar, elicitar y negociar significados, que son condición de la integración, es decir, de la construcción conjunta de aprendizaje. La integración se constituye por la síntesis de los aportes hechos por sus compañeros, con la de otras fuentes y con sus propias reflexiones.

Finalmente, sobre la base de este resultado más global, se ofrecen algunas reflexiones en un intento de aproximación conceptual y metodológica a la respuesta que reclama la pregunta central de esta investigación: en cuanto a cuáles son las interacciones de la cooperación que favorecen el aprendizaje durante tareas que exigen la construcción conjunta de conocimientos que pueden constituir indicadores para su evaluación en entornos virtuales de aprendizaje asincrónicos y escritos.

Como se ha manifestado anteriormente, la construcción de significado conjunto es esencial para ser considerado como un proceso de aprendizaje cooperativo, por ello las interacciones que favorecen el aprendizaje durante estas tareas, son en las que se presenta la implicación grupal y además la regulación grupal, en donde cada integrante sepa la ficha esencial que juega en el proceso y además ayude a mantener un buen ambiente en el que se propicie una buena construcción de conocimientos. 
El resultado de los casos analizados apunta que los factores que favorecen el aprendizaje durante tareas que exigen la construcción conjunta de conocimientos, se relacionan con la elicitación, la propuesta de ideas para la negociación así como los conocimientos previos y la la valoración de los aportes para su posterior integración. Estos supuestos son similares a los encontrados en diversas investigaciones que se toman como referencia para este estudio.

Además, y como un resultado circunstancial de este estudio, es necesario advertir que la cantidad de intervenciones en una interacción no significa necesariamente construcción de conocimiento. Una vez más, dependiendo de la fase de cooperación en la que el grupo se encuentre, se puede evidenciar que clase de interacciones se pueden estar produciendo. Aunque se hayan encontrado modelos, en los que se puede observar cómo se mueven o deberían mover las dimensiones de la regulación, es importante analizar la calidad de las interacciones en las fases.

La calidad de las interacciones solo puede ser juzgada al estudiar los mensajes que la constituyen, y de observar la función y la intención de cada una de las unidades temáticas que constituyen cada mensaje. Además, éstos deben ser estudiados en función de lo que el grupo supone querer/intentar construir en esa interacción.

Las interacciones que favorecen al aprendizaje cooperativo, se componen de intervenciones en la que los miembros del grupo se muestran abiertos, así como constató el estudio de Vauras (2003) a aprender, a escuchar, a ayudar y apoyar a su grupo o al que lo necesite.

La flexibilidad de la participación también es un aspecto importante en la construcción conjunta, puesto que se debe ser consciente de las diferentes perspectivas y alternativas que pueden haber para dar solución a un tema, y esto se puede conseguir mediante el diálogo, la negociación conjunta.

La buena disposición demostrada por los integrantes del grupo, favorece al buen clima de trabajo cooperativo, que se ve amenazado por el medio virtual si éste no se mantiene bien y vivo. La disposición comprende que los estudiantes muestren y compartan emociones positivas, como enfatiza Volet et. al (2008). Para concluir, cabe pensar que convertir la evaluación en un proceso que apoye la enseñanza y el aprendizaje puede resultar además motivador y convertirse en fuente de satisfacciones, tanto para los docentes como para los estudiantes que se ven animados a una activa participación en procesos formativos de interés común. Para lograrlo, parece necesario contar con los alumnos y comprometerse y 
comprometerlos, poco a poco, en una participación constructiva y ventajosa para el aprendizaje.

Se coincide plenamente con la afirmación de Marcelo et. al (2008) respecto al inaplazable propósito de incentivar las prácticas educativas que permitan a los estudiantes mejorar sus competencias para aprender a través del intercambio y la colaboración. "Si bien la cooperación, en un mundo esencialmente individualista, es un tanto difícil de lograr, al menos de modo espontáneo, es fundamental que dentro de los cursos e-learning se considere un modelo pedagógico que de manera intencionada y metodológica contemple el desarrollo de actividades que preparen a la comunidad educativa (participantes) para una comunidad colaborativa, esto es, una comunidad productiva, en continuo crecimiento".

\section{Perspectivas de investigación}

Como cualquier trabajo de iniciación a la investigación, este que se presenta no es definitivo ni responde exhaustivamente a las preguntas formuladas. Es por ello que se considera prudente expresar algunos comentarios bajo el rótulo de "perspectivas".

Ante todo, para definir con mayor precisión los indicadores de utilidad para evaluar las contribuciones de la regulación a la construcción del aprendizaje, se hace necesario valorar con más detenimiento las interacciones en cada fase cooperativa. Este "zoom" permitiría precisar las correspondencias entre las diversas estrategias de regulación que implementan los estudiantes para realizar la actividad de estudio a lo largo del proceso de aprendizaje cooperativo mientras elaboran los argumentos de sus ideas (nuevos conocimientos).

A su vez, esta observación más focalizada podría ayudar a clarificar el modelo que se ha presentado en esta investigación para fines educativos más prácticos, como por ejemplo, tomarlo como referencia para elaborar pautas de apoyo a las actividades o como pistas para elaborar mensajes de ayuda (feedback) que ayuden a los estudiantes a regular su participación durante la realización de la tarea cooperativa.

Asimismo sería conveniente considerar la opinión de los docentes sobre esta propuesta para orientarnos en la definición de indicadores que pueden ser de utilidad para la evaluación del proceso y de los resultados de aprendizaje de los estudiantes.

Por último, se piensa que este trabajo es sólo una aproximación conceptual y empírica, que si bien permitió corroborar las hipótesis elaboradas como punto de partida, su aplicación requiere aún de estudios más amplios que permitan contrastar estas observaciones en otras 
actividades cooperativas, con la participación de otros docentes y otros estudiantes. Esto obedece a las limitaciones metodológicas del estudio de casos, no obstante para esta investigación nos parece una decisión pertinente.

Así, nos parece que para dar validez y continuidad a esta investigación la pregunta de investigación siguiente sería cuestionar si, efectivamente, intervenciones educativas de los docentes, ya sea para la evaluación del aprendizaje o para pautarlo, conducen realmente a la mejora de la cooperación y por consecuencia, a la construcción conjunta de conocimientos durante la discusión en tareas de aprendizaje con demandas argumentativas en entornos virtuales de comunicación asincrónica y escrita.

Se espera que que este trabajo sea una ayuda y un incentivo, en el camino hacia la innovación y mejora de las prácticas docentes en universidades virtuales. 


\section{Bibliografía}

Alvarez, I. y Guasch, T. (2006). Diseño de estrategias interactivas para la construcción de conocimiento profesional en entornos virtuales de enseñanza y aprendizaje. RED Revista de Educación a Distancia. Publicación en línea. Murcia (España). Año V. Número 14.31 de Marzo, 2006 http://www.um.es/ead/red/14/

Anderson, R. C., Nguyen-Jahiel, K., McNurlen, B., Archodidou, A., Kim, S., Reznitskaya, A.,et al. (2001). The snowball phenomenon: Spread of ways of talking and ways of thinking across groups of children. Cognition and Instruction, 19(1), 1-46.

Angeli, C., Valanides, N., \& Bonk, C. J. (2003). Communication in a web-based conferencing system: The quality of computer-mediated interactions. British Journal of Educational Technology, 34(1), 31-43.

Alvarez, I. (2005). Evaluación como situación de aprendizaje o evaluación auténtica. Perspectiva Educacional, 45, 45- 67.

Alvarez, I. (2008). La coevaluación como alternativa para mejorar la calidad del aprendizaje de los estudiantes universitarios: valoración de una experiencia. Revista Interuniversitaria de Formación del Profesorado, 63 (22,3), 127-140.

Arvaja, M., Salovaara, H., Hakkinen, P., and Jarvela, S. (2007). Combining individual and group-level perspectives for studying collaborative knowledge construction in context. Learning and Instruction, 17(4), 448-459.

Angeli, C., Valanides, N., \& Bonk, C. J. (2003). Communication in a web-based conferencing system: The quality of computer-mediated interactions. British Journal of Educational Technology, 34(1), 31-43.

Badia, A., y Álvarez, I. (2009). Enseñar y aprender en la educación superior a través de la evaluación auténtica en entornos virtuales de aprendizaje (Pp.159-186). En Castelló, M. (ed.) La evaluación auténtica en enseñanza secundaria y universitaria: investigación e innovación. Barcelona: edebé.

Barberà, E. (2004). La educación en la red. Actividades de enseñanza y aprendizaje. Paidós. España.

Bell, P. (1997). Using argument representations to make thinking visible for individuals and groups. In R. Hall, N. Miyake, \& N. Enyedy (Eds.), Proceedings of CSCL '97: The Second International Conference on Computer Support for Collaborative Learning, (pp. 10-19). Toronto: University of Toronto Press.

Belland, B. R.; Glazewski, K. D.; Richardson, J. C. (2008). A scaffolding framework to support the construction of evidence-based arguments among middle school students. Educational Technology Research and Development, 56(4), 401-422.

Biggs, J. \& K. Collis (1982). Evaluating the Quality of Learning: The SOLO Taxonomy (Structure of the Observed Learning Outcome). New York, Academic Press. 
Biggs, J. (1995). Assessing for learning: Some dimensions underlying new approaches to educational assessment. The Alberta Journal of Educational Research, 41(1), 1-17.

Boakaerts, M. \& Minnaert, A. (2006). Affective and motivational outcomes of working in collaborative groups. Educational Psychology, 26 (2), 187-208.

Bodrova, E. (2006). Developing self-regulation: the Vygotskian view. Academic Exchange Quarterly. En: http://www.articlearchives.com/education-training/education-systemsinstitutions-early/1604589-1.html

Byman, A., Järvelä, S. i Häkkinen, P. (2005). What is reciprocal understanding in virtual interaction?. Instructional Science, 33, 121-136.

Campos, M. (2004). A Constructivist method for the analysis of networked cognitive communication and the assessment of collaborative learning and knowledge-building. Journal of Asynchronous Learning Networks, 8(2). Artículo en línea. En http://www.aln.org/publications/jaln/v8n2/pdf/v8n2_campos. Pdf (Fecha consulta 30/05/2009).

Casanova, M. (2008). Aprendizaje cooperativo en un contexto virtual universitario de comunicación asincrónica: un estudio sobre el proceso de interacción entre iguales a través del análisis del discurso. Tesis Doctoral. Programa de Doctorado Psicología de la comunicación: interacción social y desarrollo humano. Departamento de Psicología Básica, Evolutiva y de la Educación. Universidad Autónoma de Barcelona.

Coll, C. (2003) Psicología de la educación. Barcelona: Editorial UOC

Cohen, L., Manion, L., \& Morrison, K. (2007). Research Methods in Education (6th Edition). Oxon: Routledge.

Davies, A. \& Dalgarno, B. (2009). Learning fire investigation the clean way: The virtual experience. Australasian Journal of Educational Technology, 25(1), 1-13.

Davis, B. \& Brewer, J. (1997). Electronic Discourse. Linguistic Individuals in VirtualSpace. New York, SUNY Press.

Dawes, L. (2008). The Essential Speaking and Listening: Talk for learning at KS2. London: Routledge.

Dillenbourg P. (1999) What do you mean by collaborative learning? In P. Dillenbourg (Ed) Collaborative-learning: Cognitive and Computational Approaches (pp.1-19). Oxford: Elsevier.

Dillenbourg, P., \& Fischer, F. (2007). Basics of Computer-Supported Collaborative Learning. Zeitschrift für Berufs- und Wirtschaftspädagogik, 21, 111-130.

Dillenbourg, P., Schneider, D. K. \& Synteta, P. (2002). Virtual learning environments. In A. Dimitracopoulou (Ed), Proceedings of the 3rd Hellenic Conference on Information \& Communication Technologies in Education (pp. 3-18). Kastaniotis Editions, Greece. 
Duffy, T., Dueber, B. \& Hawley, C. (1998). Critical thinking in a distributed environment: A Pedagogical base for the design of conferencing systems. En Bonk, C. y King, K. Electronic Collaborators. New Jersey, Lawrence Erlbaum Ass, pp. 51-78.

Durán, D. y Monereo, C. (2005) Styles and sequences of cooperative interactions in fixed and reciprocal peer tutoring. Learning \& Instruction, 15; 179-199.

Fischer, F., Bruhn, J., Gräsel, C., \& Mandl, H. (2002). Fostering collaborative knowledge construction with visualization tools. Learning and Instruction, 12, 213-232.

Garrison, D., Anderson, T. \& Archer, W. (2000). Critical thinking in a text-based environment: computer conferencing in Higher Education. The Internet and Higher Education, 2(2-3), 87-105.

Garrison, D. y Anderson, T. (2005). El e-learning en el siglo XXI: investigación y práctica (trad. de A. Fuentes). Barcelona: Octaedro. [V.O.: E-learning in the 21 st century. RoutledgeFalmer, 2003].

Gibson, C. (2001). Me and us: Differential relationship among goal setting, training, efficacy at the individual and team level. Journal of Organizational Behaviour, 22, 782-802.

Green, S. (2001). A study of the effects of content and structural support in writing tasks. Paper presented at the 12th European Conference on Reading. Dublin, Ireland. July 1-4 2001.

Gulikers, J., Bastiaens, Th., \& Martens, R. (2005). The surplus value of an authentic learning environment. Computers in Human Behavior, 21(3), 509-521.

Gunawardena, C., Lowe, C., and Anderson, T. (1997). Analysis of a global online debate and the development of an interaction analysis model for examining social construction of knowledge in computer conferencing Journal of Educational Computing Research, 17(4), $395-431$.

Hadwin, A.F., Wozney, L., \& Pontin, O. (2005). Scaffolding the appropriation of selfregulatory activity: A sociocultural analysis of changes in student-teacher discourse about a graduate research portfolio. Instructional Science, 33 (5-6), 413-450.

Hara, N., Bonk, C. \& Angeli, C. (2000). Content analysis of online discussion in an applied Educational Psychology. Instructional Science, 28(2), 115-152.

Harris, K., \& Graham, S. (2006). Improving the writing, knowledge, and motivation of struggling young writers: Effects of self-regulated strategy development with and without peer support. American Educational Research Journal, 43(2), 295-340.

Henri, F. (1992). Computer conferencing and content analysis. In A. R. Kaye (Eds.), Collaborative learning through computer conferencing: The Najaden papers (pp. 115 136). New York: Springer.

Hung, D., \& Der-Thanq, Ch (2001). Situated cognition, Vygotskian thought and learning from the communities of practice perspective: implications for the design of web-based Elearning. Educational Media International, 38(1), 3-12. 
Jarvela, S. \& Hakkinen, P. (2002). Web-based Cases in Teaching and Learning - the Quality of Discussions and a Stage of Perspective Taking in Asynchronous Communication. Interactive Learning Environments, 10(1), 1-22.

Järvela, S., Jarvenoja, H. \& Veermans, M. (2008). Understanding the dynamics of motivation in socially shared learning. International Journal of Educational Research, 47(2), 122135 .

Johnson, D. \& Johnson, R. (1999). Aprender juntos y solos. Aprendizaje cooperativo, competitivo e individualista (trad. de M. Wald). Buenos Aires: Aique. [V.O.: Learning together and alone: cooperative, competitive and individualistic learning.

Kang, I. (1998). The use of computer-mediated communication: Electronic collaboration and interactivity. En C. J. Bonk \& K. King (Eds.), Electronic Collaborators (pp. 315-337). Mahwah, NJ: Lawrence Erlbaum.

León, O. Montero, I. (2003) Métodos de investigación en psicología y Educauón. Mc Graw Hill. Madrid. 120-169.

Marcelo, C., Lucero, M., Schalk, A. \& Gago, M. (2008). Aprendiendo la colaboración en los ambientes virtuales de aprendizaje. Congreso Virtual Iberomericano de Calidad en $\begin{array}{lllll}\text { Educación } & \text { a } & \text { Distancia } & \text { Edu Q } & 2008\end{array}$ http://216.75.15.111/ joomlas/eduqa2008/images/ponencias/eje_tematico_4/4_40_Apren diendo_la_colaboracion_Garcia_Lucero_Schalk_Quintanar_Gago_Nieto.p.pdf

Martin, J. \& McLellan, A. (2008). The educational psychology of self-regulation: a conceptual and critical analysis. Studies in Philosophy and Education, 27(6), 433-448.

Mathur, S. \& Murray, T. (2005). Authentic Assessment Online: A Practical and theoretical Chalenge in Higher Education. (Pp. 238- 258). En Williams, D. Online assessment, Measurement and Evaluation: Emerging Practices. Idea Group Pub.

Mauri, T, Colomina, R. \& Gispert, I. (2009). Diseño de propuestas docentes con TIC para la enseñanza de la autorregulación en la Educación Superior. Revista de Educación, 348, 377-399.

Mercer, N. \& Littleton, K. (2007) Dialogue and the Development of Children's Thinking. London: Routledge.

Mercer, N. (2004). Sociocultural discourse analysis: analysing classroom talk as a social mode of thinking. Journal of Applied Linguistics, 1, 2, 137-168.

Meyer, D. \& Turner, J. (2002).Using instructional discourse analysis to study the scaffolding of student self regulation. Educacional Psycologist, 37, 17-25.

Monereo, C. y Pozo, J.I. (2003). La universidad ante la nueva cultura educativa. Enseñar y aprender para la autonomía. Madrid: Síntesis. 
Murphy, E. (2004. An instrument to support thinking critically about critical thinking in online asynchronous discussions. Australasian Journal of Educational Technology, 20(3), 295315.

Onrubia, J., Colomina, R. \& Engel, A. (2007). Los entornos virtuales de aprendizaje basados en el trabajo en grupo y el aprendizaje colaborativo. En C. Coll \& C. Monereo (Eds.) (2007). Psicología de la Educación virtual. Aprender y enseñar con las Tecnologías de la Información y la Comunicación (pp. 233-252). Madrid: Morata.

Osborn, L. \& Nelly, J. (2003): Performance Assessment in online learning. Conference on Distance Teaching and Learning. 19 Annual Conference on Distance Teaching and Learning, August 14-15. WTCS Foundation - WIDS. [Fecha de consulta: 30/05/2009]. Disponible en www.uwex.edu/disted/conference/Resource library/proceedings/03 03.pdf

Paris, S. \& Paris, A. (2001). Classroom applications of research on self-regulated learning. Educational Psychologist, 36(2), 89-101.

Patton, M. A. (1990). Qualitative evaluation and research methods (2nd ed.). Newbury Park, CA: Sage.

Patrick, H. \& Middleton, M.J. (2002). Turning the Kaleidoscope: What we see when selfregulated learning is viewed with a qualitative lens. Educational Psychologist. 37(1), 27 39.

Pintrich, P. (2000). The role of goal orientation in self-regulated learning. In M. Boekaerts, P. R. Pintrich,\&M. Zeidner (Eds.), Handbook of self-regulation: Theory, research, and applications (pp. 452-502). San Diego, CA: Academic.

Pintrich, P. \& Zusho, A. (2002). The development of academic self-regulation: The role of cognitive and motivational factors. En A. Wigfield y J.S. Eccles (Eds.), Development of achievement motivation. San Diego: Academic Press.

Reusser, K. (2001). Co-constructivism in educational theory and practice. In N. J. Smelser, P. Baltes, \& F. E. Weinert (Eds.), International encyclopedia of the social and behavioral sciences (pp. 2058-2062). Oxford, UK: Pergamon/Elsevier Science.

Reznitskaya, A., Kuo, L.-J., Glina, M., \& Anderson, R. (2008). Measuring argumentative reasoning: What's behind the numbers? Learning and Individual Differences, 19(2), 219-224.

Rogoff, B. (1990). Apprenticeship in thinking: Cognitive development in social context. New York: Oxford University Press.

Rodriguez Illera, J. (2001). Aprendizaje colaborativo en entornos virtuales. Anuario de Psicología, 32 (2), 63-75.

Roschelle, J., \& Teasley, S. (1995). The construction of shared knowledge in collaborative problem solving. In C. O’Malley (Ed.), Computer-supported collaborative learning (pp. 69-197). Berlin: Springer. 
Salomon, G. (1992). What does the design of effective CSCL require and how do we study its effects? SlGCUE Outlook, Special Issue, 2,1 (3), 62-68.

Salonen, P., Vauras, M., \& Efklides, A. (2005). Social interaction - What can it tell us about metacognition and coregulation in learning? European Psychologist, 10(3), 199-208.

Schunk, D. H., \& Zimmerman, B. J. (1994). Self-regulation of learning and performance: Issues and educational applications. Hillsdale, NJ: Lawrence Erlbaum Associated, Inc.

Scott, P., Mortimer, E., \& Aguiar, O. (2006). The tension between authoritative and dialogic discourse: A fundamental characteristic of meaning making interactions in high school science lessons. Science Education, 90 (4), 605-631.

Shotsberger, P. (2001): Classifying forms of synchronous dialogue resulting from Webbased teacher professional development. Communication presented to SITE, Orlando,USES.

Swan, K., Shen, J., \& Hiltz, S. R. (2006). Assessment and Collaboration in Online Learning. Journal of Asynchronous Learning Networks, 10 (1), 45-62.

Teasley, S. (1997). Talking about reasoning: How important is the peer in peer collaboration? In L. B. Resnick, R. Säljö,C. Pontecorvo, \& B. Burge (Eds.), Discourse, tools and reasoning: Essays on situated cognition (pp. 361-384). Berlin: Springer.

Vauras, M., Iiskala, T., Kajamies, A., Kinnunen, R., \& Lehtinen, E. (2003). Shared-regulation and motivation of collaborating peers: a case analysis. Psychologia: An International Journal of Psychology in the Orient, 46, 19-37.

Volet, S., Summers, M., Thurman, J., (2009). High-level co-regulation in collaborative learning: How does it merge and how is it sustained, Learning and Instruction, 19 (2), 128-143

Vygotsky, L. (1979) Consciousness as a problem in the psychology of behaviour. Soviet Psychology 17, 3-35.

Weinberger, A. \& Fischer, F. (2006). A framework to analyze argumentative knowledge construction in computer-supported collaborative learning. Computers \& Education, 46, $71-95$.

Weinberger, A., Stegmann, K. \& Fischer, F. (2007). Knowledge convergence in collaborative learning: Concepts and assessment. Learning and Instruction , 17, 416-426.

Wegerif R. (2001). Applying a Dialogical Model of Reason in the Classroom. En Joiner, R, Faulkner, D Miell, D. and Littleton, K. (eds.) Rethinking Collaborative Learning, Free Association Press.

Wegerif, R., Mercer, N., and Dawes, L. (1999) From social interaction to individual reasoning: an empirical investigation of a possible socio-cultural model of cognitive development. Learning and Instruction. 493-516.

Wertsch, J. V. (1991) Voices of the mind: A sociocultural approach to mediated action. Cambridge, MA: Harvard University Press. 
Whipp, J. \& Chiarelli, S. (2004). Self-regulation in a web-based course: A case study. Educational Technology Research and Development, 52(4), 5-22.

Wigings, G. (1990). The case for authentic assessment. ERIC Digest, No. ED328611.

Winn, W. (2002). Current trends in educational technology research: The study of learning environments. Educational Psychology Review, 14 (3), 331-351.

Winters, F, Greene, J. \& Costich, C. (2008). Self-regulation of learning within computer-based learning environments: a critical analysis. Educational Psychology Review, 20, 429-444.

Vighnarajah, Wong, Su Luan \& Abu Bakar, K. (2009). Qualitative findings of students' perception on practice of self-regulated strategies in online community discussion. Computers \& Education, 53 (1), 94-103.

Zhu, E. (1996). Meaning negotiation, knowledge construction, and mentoring in a distance learning course. Paper presented at the Proceedings of Selected Research and Development Presentations at the 690ational Convention of the Association for Educational Communications and Technology, Indianapolis. http://www.eric.ed.gov/ERICDocs/data/ericdocs2sq1/content_storage_01/0000019b/80/14 la1/52.pdf

Zimmerman, B. (1995). Self-regulation involves more than metacognition: A social cognitive perspective. Educational Psychologist, 30 (4), 217-221.

Zimmerman, B. (1997). Becoming a Self-Regulated Writer: A Social Cognitive Perspective. Contemporary Educational Psychology, 22, 73-101.

\section{Software para el análisis de los datos}

Atlas.ti GmbH (1993-2002). Atlas.ti - The Knowledge Workbench (Version 6.0.21) [Computer software]. Berlin, Germany: Atlas.ti GmbH. Fuente: http://www.atlasti.de/

QSR (1999). Nvivo 8 (Version 8) [Computer software]. Victoria, Australia: Qualitative Solutions and Research. Fuente: http://www.qsrinternational.com/ 


\section{Anexo A Mapa conceptual Teoría de Vygotsky}

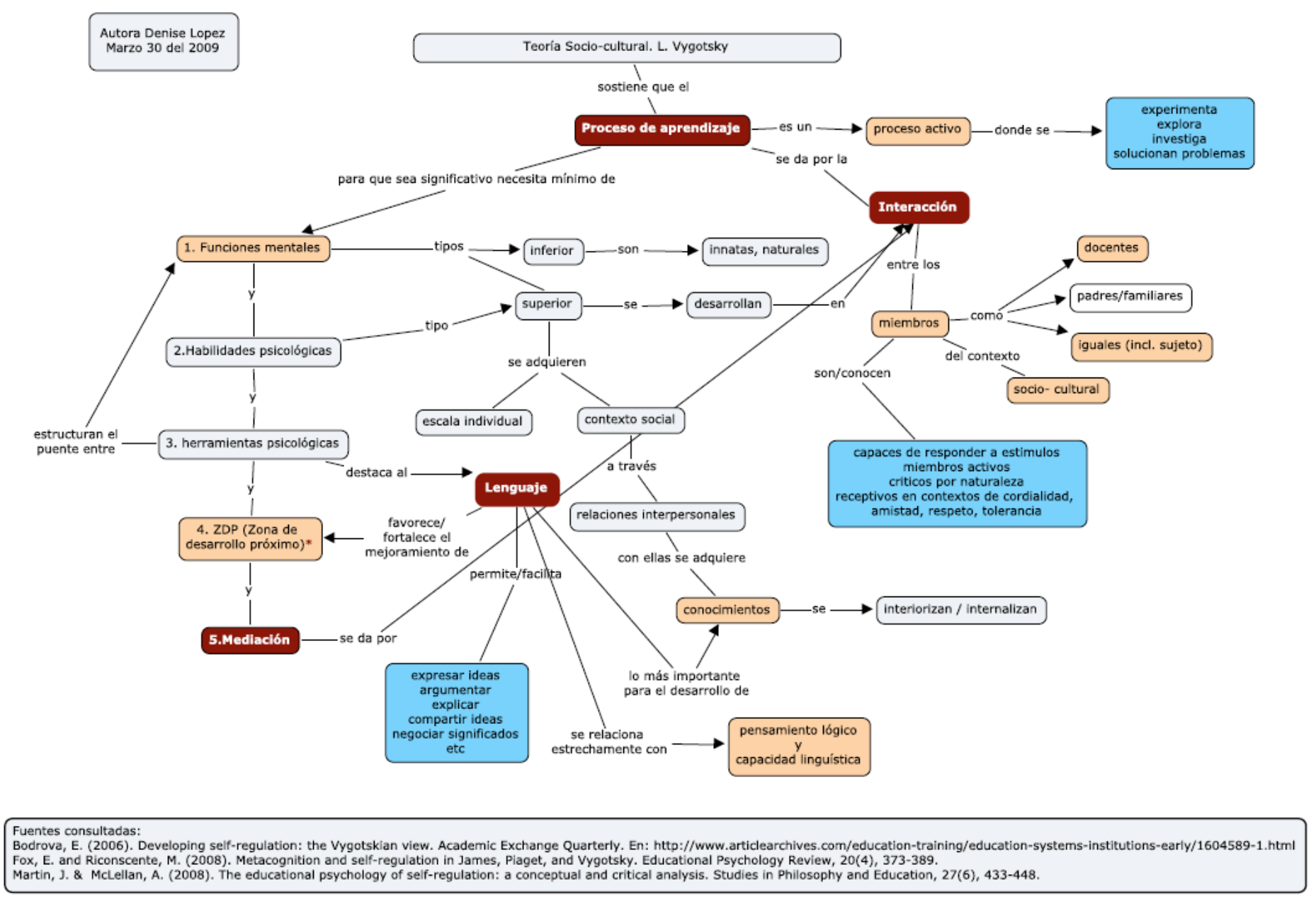

Fig. 10 


\section{Anexo B Cronograma de trabajo}

\section{Cronograma de trabajo}

\begin{tabular}{|c|c|c|}
\hline Fases & Tareas & Fecha \\
\hline $\begin{array}{l}\text { Fase I: Propuesta } \\
\text { de investigación } \\
\text { Definir y } \\
\text { comunicar el } \\
\text { tema de } \\
\text { investigadón } \\
\text { (enviar a foro del } \\
\text { seminario) }\end{array}$ & $\begin{array}{l}\text { - Breve curríaulum personal (un } \\
\text { párrafo) } \\
\text { Temática a que se referirá la } \\
\text { investigadión } \\
\text { - Especialidad a que corresponde } \\
\text { - Linea de investigación a que la que se } \\
\text { vincula (entre las propuestas) } \\
\text {. Justificación de la elección temática }\end{array}$ & Febrero \\
\hline $\begin{array}{l}\text { Fase II (a) Diseño } \\
\text { de la investigación }\end{array}$ & $\begin{array}{l}\text { Concretar el objeto y objetivo de la } \\
\text { investigadón } \\
\text { Identificar las cuestiones de } \\
\text { investigadión que pretende } \\
\text { responder (Preguntas dentíficas que } \\
\text { guían el trabajo) } \\
\text { Definir objetivo y tareas } \\
\text { Explorar antecedentes empíricos y } \\
\text { referentes concep tuales } \\
\text { Distinguir definiciones básicas. } \\
\text { Operativizar conceptos y/o } \\
\text { categorías clave. } \\
\text { Definir presupuestos y resultados } \\
\text { esperados con la investigación } \\
\text { Definir cuestiones metodológicas } \\
\text { (contexto, participantes, técnicas y } \\
\text { procedimientos) }\end{array}$ & Marzo \\
\hline $\begin{array}{l}\text { Fase II (b) } \\
\text { Desarrollo de la } \\
\text { investigación }\end{array}$ & $\begin{array}{ll} & \text { Elaborar el marco conceptual de } \\
& \text { referencia } \\
\text { - } & \text { Recopilar y analizar datos } \\
\text { - } & \text { Discusión de los resultados. } \\
\text { - } & \text { Conclusiones y perspectiva de la } \\
\text { investigación }\end{array}$ & Abril-Mayo \\
\hline $\begin{array}{l}\text { Fase III: Defensa } \\
\text { del Trabajo final } \\
\text { de investigación }\end{array}$ & $\begin{array}{l}\text { Elaborar presentación para la } \\
\text { defensa pública del trabajo } \\
\text { (informe final y Power Point (u otro } \\
\text { editor) - } 10 \text { diapositivas } \\
\text { Estructura del informe: La longitud } \\
\text { máxima del TFI será de } 50 \text { páginas, } \\
\text { si se trata de una memoria / } \\
\text { informe de investigación. En caso } \\
\text { de que se trate de una contribución } \\
\text { a una revista o congreso dentíficos } \\
\text { (peer review), la extensión se } \\
\text { tendrá que ajustar a los } \\
\text { requerimientos propios de la } \\
\text { publicación. } \\
\text { La estructura del TFI ( ver pág. } 11 \text { y } \\
\text { siguientes) }\end{array}$ & Junio \\
\hline
\end{tabular}

Fig. 11 


\title{
Anexo C Entrevista inicial a la docente de la asignatura
}

\author{
A. En cuanto a la: Tarea \\ 1. Describa las características de las actividades 2 y 3 a realizar por los alumnos.?
}

La actividad 2 consiste en un debate para profundizar en el contenido del curso. La tarea consiste en evaluar críticamente, la planificación del proceso en el estudio de un caso de referencia, a fin de extraer conclusiones en relación a exigencias, logros, dificultades, riesgos y retos que suponen la introducción de las TIC en los procesos formativos. Como resultado de un debate en grupo, basado en el estudio a fondo de la documentación disponible sobre un caso real, los estudiantes deben escribir conjuntamente las valoraciones críticas respecto al proyecto que evalúan.

En la tercera actividad, sobre la base de la segunda, se focaliza una faceta crítica del proyecto de innovación docente que han evaluado en la actividad 2 (objetivos, acciones, recursos, procedimientos, etc.) con el objetivo de proponer alternativas a las actuaciones de la institución que resuelvan o compensen las dificultades que se señalan. Es una actividad más práctica y productiva, de aplicación de conocimientos. También se realiza en grupos, habitualmente los mismos que la precedente, aunque tienen la opción de cambiar de grupo porque debe seguir siendo una actividad de aprendizaje vinculada a intereses profesionales de los estudiantes. Esto deben explicitarlo.

B. En cuanto al: Profesor

2. Cómo se les clara a los estudiantes, los criterios, las formas de actuación y de discurso que se esperan de ellos en un trabajo cooperativo?

A través de una pauta más detallada que la guía o plan de estudio que se envía a todos como documento adjunto, en mensaje en el tablón del aula. También reciben precisiones sobre la marcha, llamados de atención sobre las cuestiones a discutir y sobre las estrategias para el trabajo en grupo.

3. Cómo se establecerán las condiciones iniciales de la situación en relación con la composición de los grupos y el tipo de tareas a proponer para su realización?

En la primera parte de la actividad deciden y justifican uno, entre tres casos, para realizar el estudio. Esta decisión está basada en conocimientos previos o intereses profesionales a corto o mediano plazo. En función de las preferencias de los estudiantes los asigno a diferentes grupos que nomino con el nombre del caso. Ej. Grupo A, etc.. Máximo 5/6 estudiantes por grupo. Si el número de estudiantes que prefiere un caso sobrepasa este número los distribuyo en dos subgrupos. Para cada grupo habilito un espacio de trabajo en el debate. Esto a través de la creación de carpetas en lugar de utilizar la herramienta para crear grupos de trabajo del campus UOC. Esta decisión se justifica porque me interesa que los estudiantes puedan seguir el curso del trabajo de otros grupos, a la vez que pueden aprender de los demás sobre los modos de trabajar también pueden ampliar sus conocimientos sobre el caso que estudian u otros.

4. Cómo se establecerán las condiciones iniciales de la situación en relación con el tipo de tareas a proponer para su realización?

Me parece que está respondido arriba.

5. Cómo se actuará para apoyar las regulaciones de las interacciones en el/los grupos?

Lo mismo.. pienso que lo respondí en la 3.

6. Qué indicadores se tendrán en cuenta para evaluar hasta qué grado se están produciendo interacciones cooperativas entre los miembros de cada grupo?

Los indicadores se basan en observación de los mecanismos de la cooperación en tareas de aprendizaje cooperativa en línea. Esto empezó a interesarme como docente y acabó siendo un interés para la investigación. Es difícil y a la vez necesario. Pienso que mi perfil profesional (psicologia) y mi experiencia de investigación en la línea de la evaluación del aprendizaje en la universidad me motivó a esto.

7. Cuáles son los momentos del desarrollo del grupo cooperativo cree que será necesaria su intervención? a)La planificación de la actividad cooperativa, b) proceso de gestión de la interacción del grupo cooperativo, c) valoración del proceso y del producto) y con qué frecuencia?

Todos, pero más atencion merece el principio, sobre todo si es la primera vez que los estudiantes trabajan en grupos en el curso. Durante el transcurso observo sistemáticamente, diariamente más de una vez. No intervengo si no veo que precisan ayuda o que me lo han solicitado explícitamente. Me interesa que gestionen sus relaciones y también el conocimiento. Generalmente, si el grupo funciona bien (coopera) encuentran mejores soluciones que las que se me pueden ocurrir a mi. Por último, la evaluación es fundamental, es el momento más estratégico para mejorar los aprendizajes. Dedico un tiempo especial para esto, ellos lo saben de antemano pero no lo estiman suficientemente hasta que no lo realizan. Llevamos a cabo una co-evaluación que viene precedida de un feedback bastante crítico tanto del producto (informe del trabajo que entrega el grupo) como del proceso que realizan (observaciíon de los indicadores de la cooperación). Envio un mensaje al tablón con este feedback global y con apoyo de una herramienta para la evaluación en línea (annotation tool), pasamos a la revisión conjunta. De este proceso los estudiantes por lo general sacan provecho, en principio 
porque tienen la oportunidad de corregir sus trabajos otra vez en formato cooperativo.

C. En cuanto a los: Grupos

1. Cuál criterio se ha seguido para la composición de los grupos? (en función de los objetivos de aprendizaje, el tipo de tarea, la experiencia previa en trabajos en grupos cooperativos, los materiales de contenido, recursos de acceso a la información o el tiempo disponible para realizar la actividad)

Esto lo contesté arriba.

\section{Anexo D Encuesta en línea}

\section{DATOS GENERALES}

Nombre:

Edad:

Perfil profesional:

Experiencias como estudiante en línea:

Experiencia en el uso de las TIC para aprender:

Avanzada (Web page, herrami entas multimedia, herramientas gráficas, LMS, etc.)

— Media (Web page, herramientas para presentaciones personalizadas, aplicaciones mul timedia)

- Básica (e mail)

1. ¿Cuáles son los principales obstáculos que enfrenta para la elaboración de textos como tarea de aprendizaje en línea?

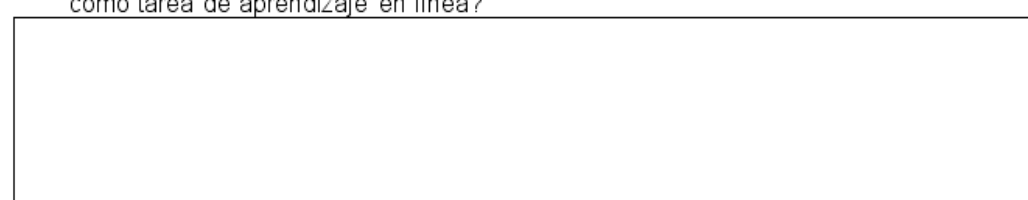

2. ¿Qué estrategias suele utilizar para afrontar personalmente las dificultades que le suponen escribir un texto como tarea de aprendizaje en línea?

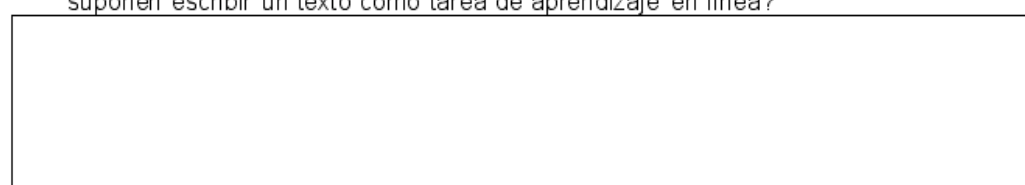

3. ¿Cuáles estrategias del docente considera útiles para ayudar a los estudiantes en la tarea de escribir en línea?

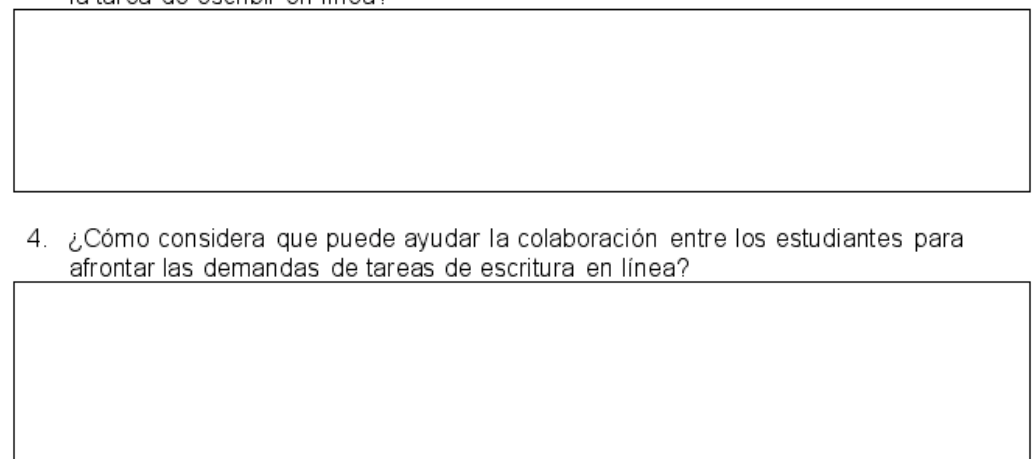

Fig. 12

Nota: El formato de la encuesta no es el original. La encuesta se realizó en línea mediante la herramienta NetQuest. 


\section{Anexo E Concordancia de categorización entre jueces}

\begin{tabular}{|c|c|c|c|c|c|c|}
\hline & Regulac & Social & & Regulac & Cogniti & \\
\hline & 11 & $\mathrm{~J} 2$ & 33 & $\mathrm{~J} 1$ & 12 & 3 \\
\hline 1 & RS & RS & RS & $\mathrm{RC}$ & $\mathrm{RC}$ & $\mathrm{RC}$ \\
\hline 2 & $\mathrm{Aev}$ & Aev & $\mathrm{Aev}$ & Ex & Ex & Ex \\
\hline 3 & $\mathrm{Aev}$ & Aev & $\mathrm{Aev}$ & Ex & Ex & Ex \\
\hline 4 & $\mathrm{QC}$ & $\mathrm{QC}$ & $\mathrm{QC}$ & Ex & Ex & Ex \\
\hline 5 & $\mathrm{Aev}$ & Aev & Aev & Ex & Ex & Ex \\
\hline 6 & RS & RS & RS & - & - & - \\
\hline 7 & ExT & EXT & Ext & Eli & Eli & Eli \\
\hline 8 & ort & ort & ort & - & - & - \\
\hline 9 & PM & PM & PM & Ex & Ex & EX \\
\hline 10 & Ort & Ort & Ort & - & - & - \\
\hline 11 & PM & PM & PM & - & - & - \\
\hline 12 & SP & $\mathrm{SP}$ & SP & - & - & - \\
\hline 13 & PM & $P M$ & $P M$ & - & - & - \\
\hline 14 & Ort & Ort & OrT & - & - & - \\
\hline 15 & RS & RS & RS & - & - & - \\
\hline 16 & ort & ort & OrT & Ex & Ex & Ex \\
\hline 17 & PM & PM & PM & - & - & - \\
\hline 18 & Ort & ort & Ort & - & - & - \\
\hline 19 & SP & SM & SP & - & - & - \\
\hline 20 & RS & RS & RS & - & - & - \\
\hline 21 & SP & $\mathrm{Sp}$ & $\mathrm{SP}$ & - & - & - \\
\hline 22 & SP & So & SP & - & - & - \\
\hline 23 & IE & $\mathrm{IE}$ & $\mathrm{IE}$ & Eli & Eli & Ell \\
\hline 24 & - & - & - & Ex & Ex & Ex \\
\hline 25 & $\mathrm{Aev}$ & $\mathrm{QC}$ & - & $10 \mathrm{C}$ & IOC & 100 \\
\hline 26 & - & - & - & Ex & Ex & EX \\
\hline 27 & EXT & EXT & ExT & NS & $10 \mathrm{C}$ & NS \\
\hline 28 & IR & $\mathrm{IR}$ & IR & Eli & Eli & Eli \\
\hline 29 & PM & $\mathrm{IE}$ & RS & Ex & Ex & Ex \\
\hline 30 & $\mathrm{AEV}$ & - & ExT & NS & NS & NS \\
\hline 31 & - & - & - & EX & Ex & Ex \\
\hline 32 & - & - & - & 100 & $\operatorname{coc}$ & Ex \\
\hline 33 & IR & IR & $\mathbb{R}$ & Eli & Eli & Eli \\
\hline 34 & - & - & - & $10 \mathrm{C}$ & Ex & Ex \\
\hline 35 & - & - & - & 100 & $\mathrm{COC}$ & Ex \\
\hline
\end{tabular}

\begin{tabular}{|c|c|c|c|c|c|c|}
\hline 36 & $\mathbb{R}$ & $\mathbb{I R}$ & $\mathbb{R}$ & $10 C$ & - & Eli \\
\hline 37 & $\mathbb{R}$ & $\mathbb{I R}$ & $\mathbb{I R}$ & - & - & - \\
\hline 38 & $\mathbb{R}$ & PM & - & 100 & $10 \mathrm{C}$ & 100 \\
\hline 39 & SM & SM & $S M$ & - & - & - \\
\hline 40 & Ort & Ort & OrT & - & - & - \\
\hline 41 & RS & RS & RS & - & - & - \\
\hline 42 & SM & SM & SM & - & - & - \\
\hline 43 & SM & SM & SM & - & - & - \\
\hline 44 & $\mathbb{R}$ & $\mathrm{IR}$ & $\mathrm{IR}$ & Eli & Eli & Eli \\
\hline 45 & $\mathbb{R}$ & $\mathrm{IR}$ & $\mathrm{IR}$ & $10 C$ & Eli & NS \\
\hline 46 & SM & SM & $\mathrm{PM}$ & - & - & - \\
\hline 47 & RS & RS & RS & - & - & - \\
\hline 48 & RS & RS & RS & - & - & - \\
\hline 49 & Ex & Ex & Ex & - & Eli & Ext \\
\hline 50 & SM & SM & SM & - & - & $\mathrm{COC}$ \\
\hline 51 & $\mathbb{R}$ & $\mathbb{R}$ & $\mathrm{IR}$ & - & Eli & $\mathrm{COC}$ \\
\hline 52 & SM & SM & SM & - & - & - \\
\hline 53 & RS & $\mathrm{IE}$ & $\mathrm{QC}$ & - & - & - \\
\hline 54 & SM & SM & SM & - & 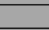 & - \\
\hline 55 & RS & RS & RS & - & - & - \\
\hline 56 & Ort & Ort & Ort & - & - & - \\
\hline 57 & SM & SM & SM & - & - & - \\
\hline 58 & $\mathbb{R}$ & $\mathbb{R}$ & $\mathrm{IR}$ & Eli & Eli & Eli \\
\hline 59 & SP & SP & $\mathrm{SP}$ & - & - & - \\
\hline 60 & RS & RS & RS & - & - & - \\
\hline 61 & $\mathbb{R}$ & $\mathbb{R}$ & $\mathrm{IR}$ & - & - & - \\
\hline 62 & SM & SM & SM & - & - & - \\
\hline 63 & RS & RS & RS & - & - & - \\
\hline 64 & SM & SM & SM & - & - & - \\
\hline 65 & $\mathrm{QC}$ & $\mathrm{QC}$ & QC & - & - & - \\
\hline 66 & SM & SM & SM & - & - & - \\
\hline 67 & RS & RS & RS & - & - & - \\
\hline 68 & SM & SM & SM & - & - & - \\
\hline 69 & RS & RS & $\mathrm{RS}$ & - & - & - \\
\hline
\end{tabular}

Tabla 17

Nota: El color amarillo en las casillas indica la discrepancia de categorías entre los jueces en las unidades temáticas evaluadas. 


\section{Anexo F Categorías del esquema argumentativo de texto escrito en tarea cooperativa*}

\section{F.1. Criterios de evaluación de textos escritos}

\begin{tabular}{|c|c|}
\hline Categorías & Descripción \\
\hline $\begin{array}{l}\text { Relevancia de las ideas } \\
\text { Ajuste de las ideas } \\
\text { respecto al objetivo de la } \\
\text { tarea }\end{array}$ & $\begin{array}{l}\text {-Ideas introductorias: Describe el propósito y/o alcance de las ideas que se } \\
\text { presentan, y se da una breve explicación o resumen de éste. También puede } \\
\text { explicar algunos antecedentes que son importantes para el posterior desarrollo } \\
\text { de las ideas centrales } \\
\text {-Ideas esenciales (IE): Posicionamientos centrales directamente relacionados } \\
\text { con la demanda argumentativa de la tarea (criterio apropiado para valorar el } \\
\text { caso) } \\
\text {-Ideas no esenciales (InE): posicionamientos centrales no relacionado con la } \\
\text { demanda argumentativa de la tarea (criterio no apropiado para valorar el caso) }\end{array}$ \\
\hline $\begin{array}{l}\text { Focalización de las ideas } \\
\text { esenciales } \\
\text { Contextualización del } \\
\text { razonamiento }\end{array}$ & $\begin{array}{l}\text { Giros ArgumentosRrelevantes (GAR): Enunciados razonados e ilustrativos } \\
\text { Giros Argumentos Circunstanciales (GAC): Enunciados superficiales poco o } \\
\text { nada ilustrativos }\end{array}$ \\
\hline $\begin{array}{l}\text { Soporte de las ideas } \\
\text { Fuente de las ideas que } \\
\text { sostienen los } \\
\text { razonamientos }\end{array}$ & $\begin{array}{l}\text { Textual }(\mathrm{Txt}) \text { : Ideas extraídas más o menos literalmente de las lecturas previas } \\
\text { Hipótesis }(\mathrm{Hp}) \text { : Enunciados referidos a acciones probables } \\
\text { Abstracción }(\mathrm{Abs}) \text { : Generalización, prescripción sobre cómo actuar o sobre } \\
\text { las consecuencias de la actuación.. } \\
\text { Contextualización }(\mathrm{Ctxt}) \text { : Enunciado que reconstruyen la situación, con } \\
\text { atención al contexto, la audiencia, etc. }\end{array}$ \\
\hline $\begin{array}{l}\text { Elaboración de las ideas } \\
\text { Esquema argumentativo } \\
\text { de las ideas esenciales }\end{array}$ & $\begin{array}{l}\text { Idea elaborada (Elab): Esquema argumentativo bien articulado: Criterio } \\
\text { valorativo + justificación + ejemplos/evidencias + } \\
\text { conclusión/contextualización de la idea esencial. } \\
\text { Idea incompleta (PElab): Giros argumentativos anclados a idea relevante } \\
\text { (criterio valorativo + justificación) pero carente de evidencias o idea } \\
\text { conclusiva contextualizada } \\
\text { Idea muy incompleta (NElab): Criterio poco claro o no justificado, } \\
\text { repeticiones de ideas, giros argumentativos desarticulados o circunstanciales. }\end{array}$ \\
\hline
\end{tabular}

Tabla 18

*Adaptado de Reznitskaya, et. al (2008).

\section{F.2. Ejemplo de la elaboración de ideas: Idea elaborada}

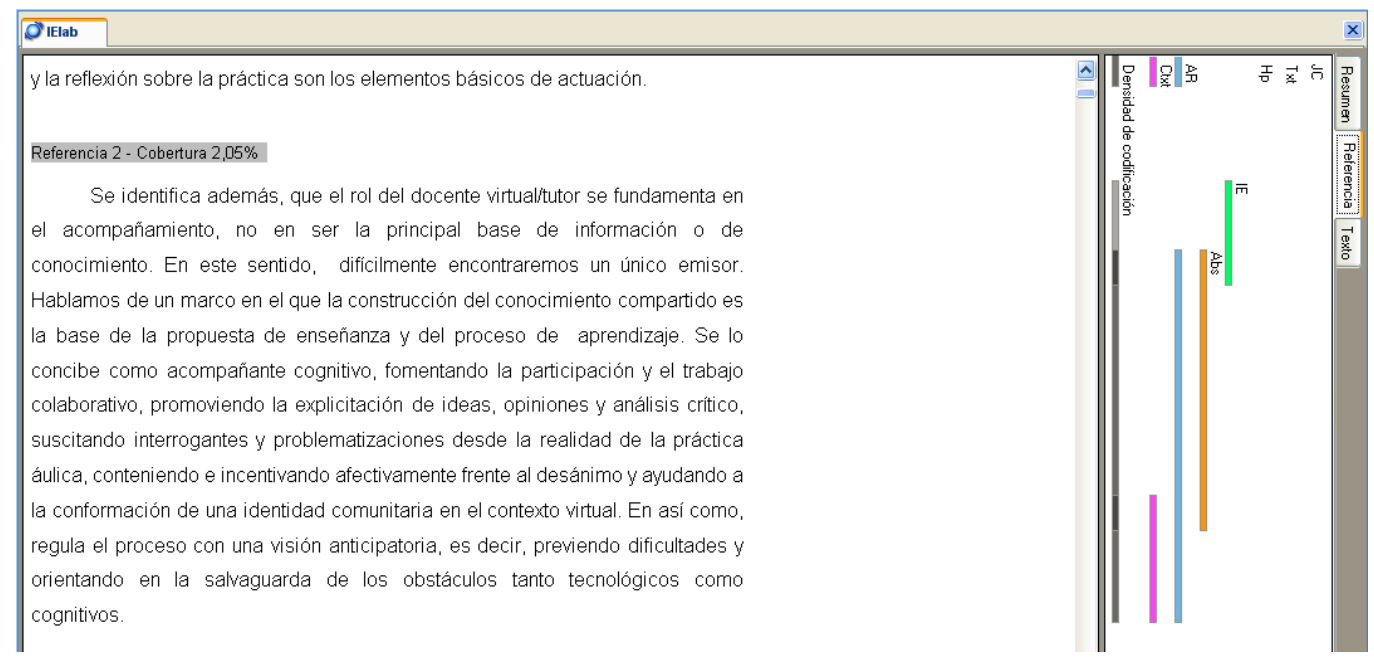

Fig. 13 


\section{F.3. Ejemplo de la elaboración de ideas: Idea incompleta}

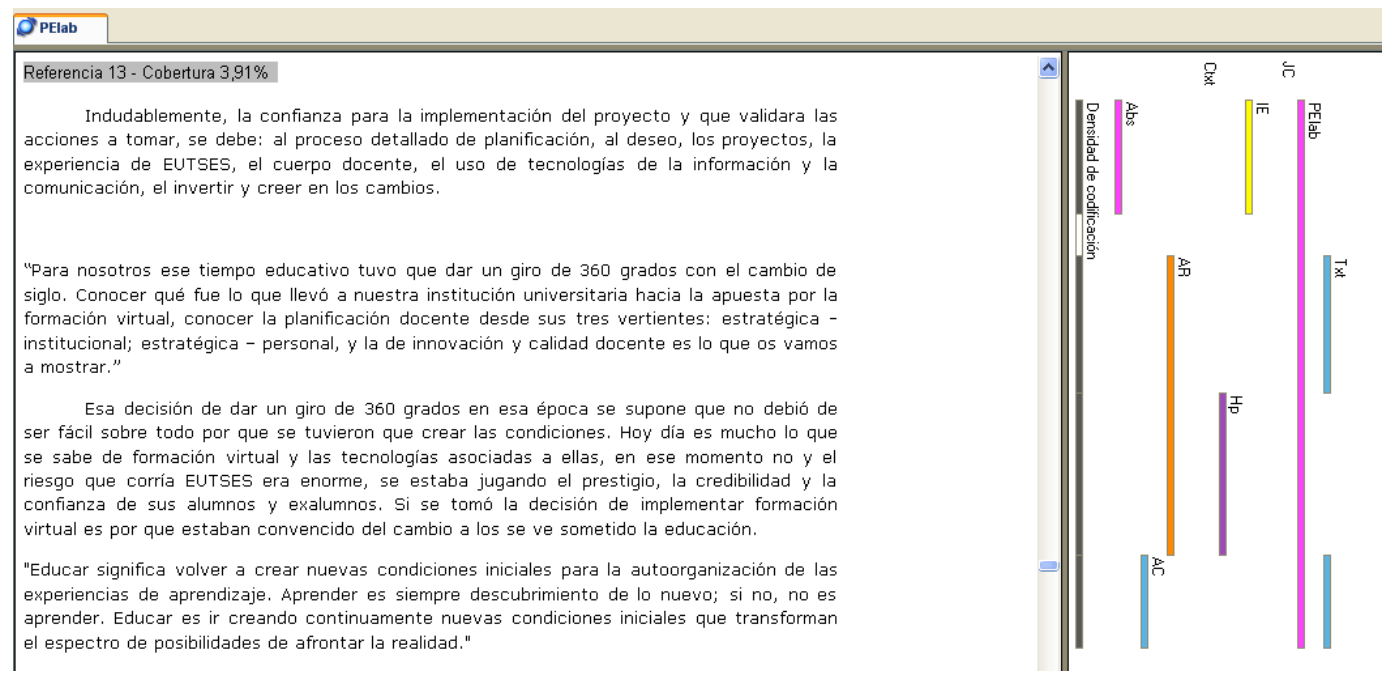

Fig. 14

\section{F.4. Ejemplo de la elaboración de ideas: Idea no elaborada}

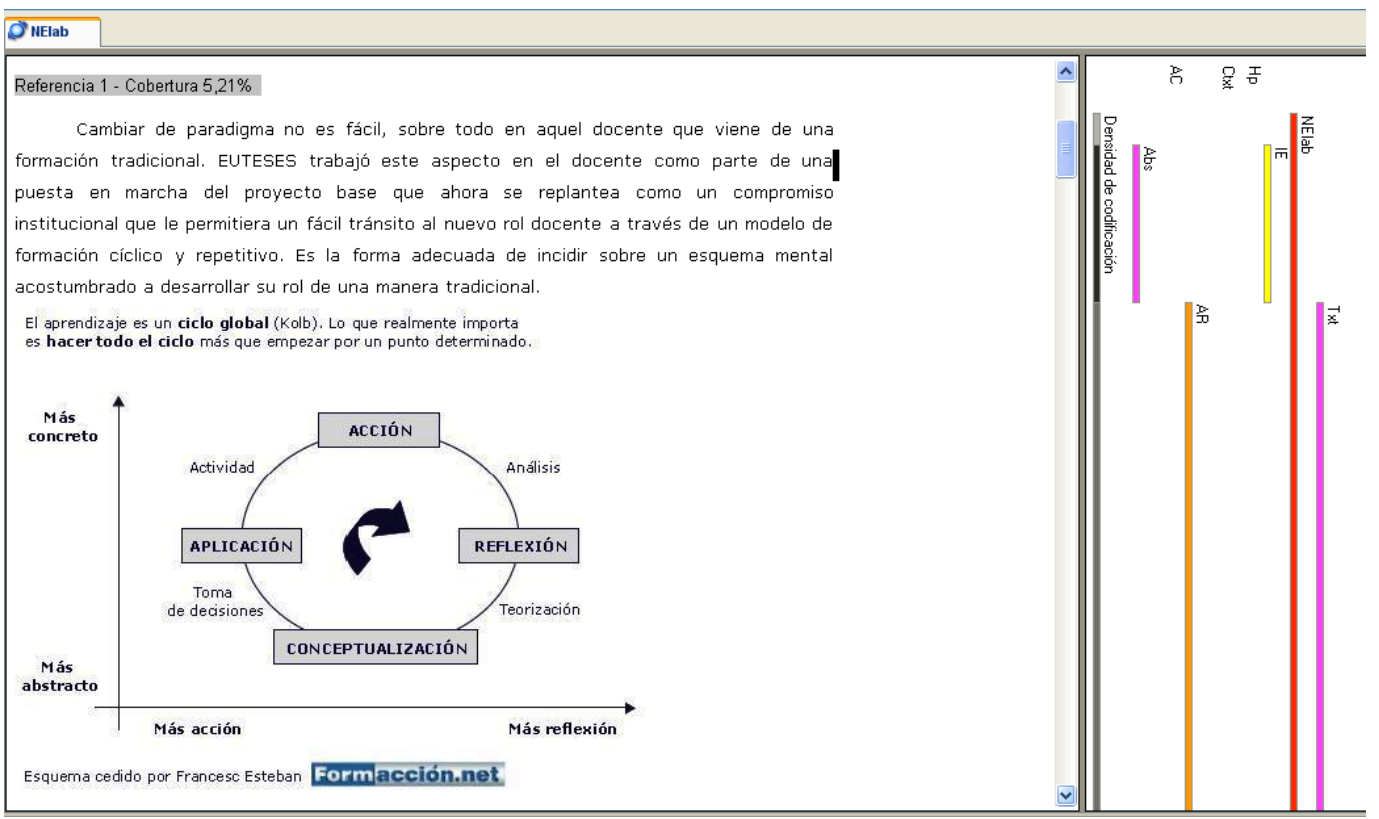




\section{Anexo G El entorno virtual de la UOC (Universitat Oberta de Catalunya)}

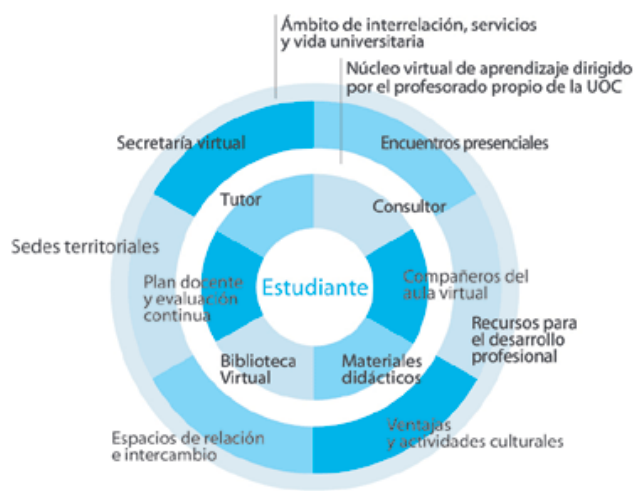

Fig. 15 Modelo Educativo de la UOC

http://www.uoc.edu/

\begin{tabular}{|c|c|c|c|c|c|c|c|}
\hline$\overline{-}$ & & ? & $\approx$ Debate & & & & Cerrar \\
\hline & & & Auditor de la cal & & & & \\
\hline ES Nue & & & $\Delta^{\text {Abrir }}$ & Responder & (2) Al remitente & t: Reenviar & 目 Historial \\
\hline Leer toc & os 10 & nsaje: & Dar por leído Order & ar Exportar & & & 힘 \\
\hline - Recil & idos & & & Expe & eriencia de apren & dizaje pe & rsonal \\
\hline vert & dos & sólo & nuevos & & & Se muestran 1 & 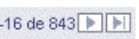 \\
\hline+ & E & $\square$ & De: Exduardo & & Debate 6. MERECE LA PENA & & $020423: 10$ \\
\hline 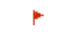 & E & $\square$ & De: Eduardo & & ALGUNO QUEDARÁ & & $102 \% 422: 59$ \\
\hline+ & E & $\square$ & De: Eduard & & debate 6 & & $102 / 0413: 34$ \\
\hline+ & E & $\square$ & De: Ana & & Debate 6 & & $102 / 0408: 42$ \\
\hline$r$ & E & $\square$ & De: Joan & & Debate 6 : Sistena para la q... & & $102 / 0401: 09$ \\
\hline p & E & $\square$ & De: Antoni & & Debate 6. & & $102: 0423.57$ \\
\hline$p$ & E & $\bar{\square}$ & De: Magda & & & & $02 / 0419: 16$ \\
\hline$p$ & E & $\square$ & De: Antonio & & Debate 6 & & $102: 0418: 10$ \\
\hline
\end{tabular}

Fig. 16 Espacio de debate del aula virtual de la UOC

http://www.uoc.edu/ 
Anexo H Resumen de las categorías de las regulación y sus descriptores.

\begin{tabular}{|c|c|c|c|c|}
\hline \multicolumn{2}{|c|}{$\begin{array}{l}\text { Dimen } \\
\text { sión }\end{array}$} & \multirow{2}{*}{\begin{tabular}{|l|} 
Código \\
Ort
\end{tabular}} & \multirow{2}{*}{$\begin{array}{l}\text { Categorías } \\
\begin{array}{l}\text { Estructurar la } \\
\text { tarea }\end{array}\end{array}$} & \multirow{2}{*}{$\begin{array}{l}\text { Descriptores } \\
\qquad \begin{array}{l}\text { Intervenciones con preguntas o propuestas sobre organización, } \\
\text { procedimiento, roles, recursos, planificación temporal, formato del texto, } \\
\text { etc. }\end{array}\end{array}$} \\
\hline \multirow{3}{*}{ 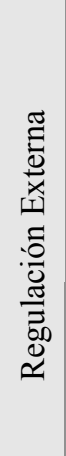 } & & & & \\
\hline & is & $\mathrm{RS}$ & $\begin{array}{l}\text { Refuerzos } \\
\text { sociales }\end{array}$ & $\begin{array}{l}\text { Intervenciones emotivas que respaldan ideas o actuaciones de otros porque } \\
\text { inciden positivamente en la cognición o en la motivación de los demás. }\end{array}$ \\
\hline & : & ExT & $\begin{array}{l}\text { Explicitar la } \\
\text { tarea }\end{array}$ & $\begin{array}{l}\text { Interacciones no argumentativas que giran alrededor del objetivo común. } \\
\text { El objetivo de la tarea se analiza, se aclara, se reformula, se repasa. }\end{array}$ \\
\hline \multirow{5}{*}{ 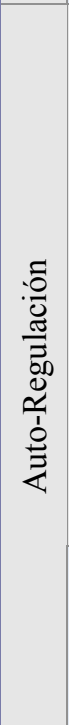 } & \multirow{4}{*}{$\begin{array}{l}\bar{\pi} \\
0 \\
\infty\end{array}$} & $\mathrm{AeV}$ & $\begin{array}{l}\text { Auto- } \\
\text { evaluación }\end{array}$ & $\begin{array}{l}\text { Intervenciones que reflejan la valoración de sus conocimientos o } \\
\text { experiencias previas que puedan contribuir a la resolución de la tarea o } \\
\text { que reflejen lo que la resolución de la tarea le aportará respecto a las } \\
\text { demandas de su contexto cotidiano. }\end{array}$ \\
\hline & & SA & $\begin{array}{l}\text { Situar el propio } \\
\text { proceso de } \\
\text { aprendizaje }\end{array}$ & $\begin{array}{l}\text { El estudiante asimila el objetivo de la tarea, lo relaciona con sus } \\
\text { conocimientos previos y valora lo que necesita para alcanzarlo. }\end{array}$ \\
\hline & & PI & $\begin{array}{l}\text { Planificación } \\
\text { individual }\end{array}$ & $\begin{array}{l}\text { El estudiante valora los recursos y tiempo disponibles para determinar su } \\
\text { colaboración y asume voluntariamente responsabilidades. }\end{array}$ \\
\hline & & MI & $\begin{array}{l}\text { Monitorización } \\
\text { de su } \\
\text { participación }\end{array}$ & Control de la gestión de su propia participación \\
\hline & ن & Ex & Exteriorizar & $\begin{array}{l}\text { Intervenciones no argumentativas que aportan información de contenido } \\
\text { textual o expresan puntos de vista sobre el contenido a tratar sin referencia } \\
\text { a contribuciones previas. }\end{array}$ \\
\hline \multirow{6}{*}{ 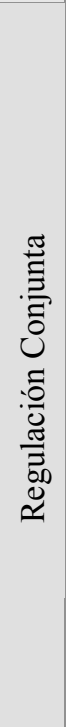 } & \multirow{3}{*}{$\begin{array}{l}\frac{\pi}{0} \\
\text { 足 }\end{array}$} & IR & $\begin{array}{l}\text { Interpelar } \\
\text { responsabilidad } \\
\text { / participación } \\
\text { de los demás }\end{array}$ & $\begin{array}{l}\text { Interacciones que solicitan la ayuda/colaboración a sus compañeros, en } \\
\text { función de la organización y realización de la tarea. }\end{array}$ \\
\hline & & PM & $\begin{array}{l}\text { Perspectiva } \\
\text { mutua }\end{array}$ & $\begin{array}{l}\text { Interacciones que comunican un acuerdo recíproco, se reconoce, se valora } \\
\text { y se refuerza una idea }\end{array}$ \\
\hline & & Ccr & $\begin{array}{l}\text { Consenso corto } \\
\text { y rápido }\end{array}$ & Interacciones que muestran conformidad o neutralidad con algo expuesto \\
\hline & \multirow{3}{*}{ 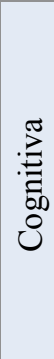 } & Eli & Elicitar & $\begin{array}{l}\text { Intervenciones que solicitan, directa o indirectamente, la reacción de otro } \\
\text { compañero(a) en función del contenido de la tarea. }\end{array}$ \\
\hline & & NS & $\begin{array}{l}\text { Negociar } \\
\text { significado }\end{array}$ & $\begin{array}{l}\text { Intervenciones argumentadas que ofrecen propuestas, alternativas y/o } \\
\text { complemento a información intercambiada, con la intención de llegar a un } \\
\text { acuerdo. }\end{array}$ \\
\hline & & IOC & $\begin{array}{l}\text { Integración } \\
\text { orientada al } \\
\text { consenso }\end{array}$ & $\begin{array}{l}\text { Intervenciones argumentadas que manifiestan consenso a partir de integrar } \\
\text { reflexiones propias con la información aportada por los demás. }\end{array}$ \\
\hline
\end{tabular}

